\title{
DERIVATION OF AN EFFECTIVE EVOLUTION EQUATION FOR A STRONGLY COUPLED POLARON
}

\author{
RUPERT L. FRANK AND ZHOU GANG
}

\begin{abstract}
Fröhlich's polaron Hamiltonian describes an electron coupled to the quantized phonon field of an ionic crystal. We show that in the strong coupling limit the dynamics of the polaron are approximated by an effective nonlinear partial differential equation due to Landau and Pekar, in which the phonon field is treated as a classical field.
\end{abstract}

\section{Introduction and main result}

1A. Setting of the problem. In this paper we are interested in the dynamics of a strongly coupled polaron. A polaron is a model of an electron in an ionic lattice interacting with its surrounding polarization field. Fröhlich [1937] proposed a quantum-mechanical Hamiltonian, given in (1-1) below, in order to describe the dynamics of a polaron. In this model the phonon field is treated as a quantum field. The Fröhlich Hamiltonian depends on a single parameter $\alpha>0$ which describes the strength of the coupling between the electron and the phonon field. Landau and Pekar [1948] proposed a system of nonlinear PDEs, see (1-8), (1-9) below, to describe the dynamics of a polaron and used this in their famous computation of the effective polaron mass (see [Spohn 1987] for an alternative approach). They treat the phonons as a classical field. The derivation of their equations is phenomenological and they do not comment on the relation between their equations and the dynamics generated by Fröhlich's Hamiltonian. Our purpose in this paper is to establish a connection between the two dynamics and to rigorously derive the Landau-Pekar equations from the Fröhlich dynamics in the strong coupling limit $\alpha \rightarrow \infty$ for a natural class of initial conditions and on certain time scales.

In order to describe this result in detail, we recall that the Fröhlich Hamiltonian acts in $\mathcal{L}^{2}\left(\mathbb{R}^{3}\right) \otimes \mathcal{F}$, where $\mathcal{L}^{2}\left(\mathbb{R}^{3}\right)$ corresponds to the electron and $\mathcal{F}=\mathcal{F}\left(\mathcal{L}^{2}\left(\mathbb{R}^{3}\right)\right)$, the bosonic Fock space over $\mathcal{L}^{2}\left(\mathbb{R}^{3}\right)$, corresponds to the phonon field. The Hamiltonian is given by

$$
p^{2}+\sqrt{\alpha} \int_{\mathbb{R}^{3}}\left[e^{-i k \cdot x} a_{k}+e^{i k \cdot x} a_{k}^{*}\right] \frac{d k}{|k|}+\int_{\mathbb{R}^{3}} a_{k}^{*} a_{k} d k,
$$

where $p:=-i \nabla_{x}$ and $x$ are momentum and position of the electron and $a_{k}$ and $a_{k}^{*}$ are annihilation and creation operators in $\mathcal{F}$ satisfying the commutation relations

$$
\left[a_{k}, a_{k^{\prime}}^{*}\right]=\delta\left(k-k^{\prime}\right), \quad\left[a_{k}, a_{k^{\prime}}\right]=0, \quad \text { and } \quad\left[a_{k}^{*}, a_{k^{\prime}}^{*}\right]=0 .
$$

MSC2010: 35Q40, 46N50.

Keywords: polaron, dynamics, quantized field. 
As mentioned before, the scalar $\alpha>0$ describes the strength of the coupling between the electron and the phonon field and will be large in our study.

To facilitate later discussions we rescale the variables, as in [Frank and Schlein 2014],

$$
x \mapsto \alpha^{-1} x, \quad k \mapsto \alpha k,
$$

and find that the Hamiltonian in (1-1) is unitarily equivalent to $\alpha^{2} \widetilde{H}_{\alpha}^{F}$, where the new Hamiltonian $\widetilde{H}_{\alpha}^{F}$, acting again in $\mathcal{L}^{2}\left(\mathbb{R}^{3}\right) \otimes \mathcal{F}$, is defined as

$$
\widetilde{H}_{\alpha}^{F}:=p^{2}+\int_{\mathbb{R}^{3}}\left[e^{-i k \cdot x} b_{k}+e^{i k \cdot x} b_{k}^{*}\right] \frac{d k}{|k|}+\int_{\mathbb{R}^{3}} b_{k}^{*} b_{k} d k .
$$

The new annihilation and creation operators $b_{k}:=\alpha^{1 / 2} a_{\alpha k}$ and $b_{k}^{*}:=\alpha^{1 / 2} a_{\alpha k}^{*}$ satisfy the commutation relations

$$
\left[b_{k}, b_{k^{\prime}}^{*}\right]=\alpha^{-2} \delta\left(k-k^{\prime}\right), \quad\left[b_{k}, b_{k^{\prime}}\right]=0, \quad \text { and } \quad\left[b_{k}^{*}, b_{k^{\prime}}^{*}\right]=0 .
$$

We emphasize the $\alpha$-dependence in (1-5).

We will discuss the dynamics generated by $\widetilde{H}_{\alpha}^{F}$ for initial conditions of the product form

$$
\psi_{0} \otimes W\left(\alpha^{2} \varphi_{0}\right) \Omega \text {. }
$$

Here, $\Omega$ denotes the vacuum in $\mathcal{F}$ and $W(f)$ denotes the Weyl operator,

$$
W(f):=e^{b^{*}(f)-b(f)}
$$

so that $W\left(\alpha^{2} \varphi\right) \Omega$ is a coherent state. This particular choice of initial conditions is motivated by Pekar's approximation [1946; 1951] to the ground state energy, which uses exactly states of this form. Pekar's approximation was made mathematically rigorous by Donsker and Varadhan [1983] (see [Lieb and Thomas 1997] for an alternative approach).

Clearly, the time-evolved state $e^{-i \tilde{H}_{\alpha}^{F} t} \psi_{0} \otimes W\left(\alpha^{2} \varphi_{0}\right) \Omega$ with $t \neq 0$ will in general no longer have an exact product structure. However, we will see that for large $\alpha$ (and $t$ of order one, or even larger) it can be approximated, in a certain sense, by a state of the product form $\psi_{t} \otimes W\left(\alpha^{2} \varphi_{t}\right) \Omega$, where $\psi_{t}$ and $\varphi_{t}$ solve the Landau-Pekar equations

$$
\begin{aligned}
i \partial_{t} \psi_{t}(x) & =\left[-\Delta+\int_{\mathbb{R}^{3}}\left[e^{-i k \cdot x} \varphi_{t}(k)+e^{i k \cdot x} \overline{\varphi_{t}(k)}\right] \frac{d k}{|k|}\right] \psi_{t}(x), \\
i \alpha^{2} \partial_{t} \varphi_{t}(k) & =\varphi_{t}(k)+|k|^{-1} \int_{\mathbb{R}^{3}}\left|\psi_{t}(x)\right|^{2} e^{i k \cdot x} d x
\end{aligned}
$$

with initial data $\psi_{0}$ and $\varphi_{0}$. Using standard methods one can show that for any $\psi_{0} \in \mathcal{H}^{1}\left(\mathbb{R}^{3}\right), \varphi_{0} \in \mathcal{L}^{2}\left(\mathbb{R}^{3}\right)$ and $\alpha>0$, the system (1-8), (1-9) has a global solution $\left(\psi_{t}, \varphi_{t}\right)$, which satisfies

$$
\left\|\psi_{t}\right\|_{\mathcal{L}^{2}\left(\mathbb{R}^{3}\right)}=\left\|\psi_{0}\right\|_{\mathcal{L}^{2}\left(\mathbb{R}^{3}\right)} \quad \text { and } \quad \mathcal{E}\left(\psi_{t}, \varphi_{t}\right)=\mathcal{E}\left(\psi_{0}, \varphi_{0}\right) \quad \text { for all } t \in \mathbb{R}
$$

with the energy

$$
\mathcal{E}(\psi, \varphi):=\int_{\mathbb{R}^{3}}|\nabla \psi|^{2} d x+\int_{\mathbb{R}^{3}}|\psi(x)|^{2} \int_{\mathbb{R}^{3}}\left(e^{-i k \cdot x} \varphi(k)+e^{i k \cdot x} \bar{\varphi}(k)\right) \frac{d k}{|k|} d x+\int_{\mathbb{R}^{3}}|\varphi(k)|^{2} d k .
$$


We refer to Lemma 2.1 and Proposition 2.2 for more details about the solution $\left(\psi_{t}, \varphi_{t}\right)$. In the original work of Landau and Pekar the equations are given in a different, but equivalent form, and we explain this connection in Section 1D.

1B. Main result. In order to prove our main result we need the following regularity and decay assumptions on the initial data. We denote by $\mathcal{H}^{m}\left(\mathbb{R}^{3}\right)$ the Sobolev space of order $m$ and by

$$
\mathcal{L}_{(m)}^{2}\left(\mathbb{R}^{3}\right):=\mathcal{L}^{2}\left(\mathbb{R}^{3},\left(1+k^{2}\right)^{m} d k\right)
$$

the weighted $\mathcal{L}^{2}$ space with norm

$$
\|\varphi\|_{\mathcal{L}_{(m)}^{2}}=\left(\int_{\mathbb{R}^{3}}\left(1+k^{2}\right)^{m}|\varphi(k)|^{2} d k\right)^{\frac{1}{2}} .
$$

Our main result will be valid under the following:

Assumption 1.1. We assume $\psi_{0} \in \mathcal{H}^{4}\left(\mathbb{R}^{3}\right)$ and $\varphi_{0} \in \mathcal{L}_{(3)}^{2}\left(\mathbb{R}^{3}\right)$ with $\left\|\psi_{0}\right\|_{\mathcal{L}^{2}\left(\mathbb{R}^{3}\right)}=1$.

A first version of our main result concerns the approximation of the reduced density matrices of $e^{-i \widetilde{H}_{\alpha}^{F} t} \psi_{0} \otimes W\left(\alpha^{2} \varphi_{0}\right) \Omega$ in the trace norm.

Theorem 1.2. Assume $\psi_{0}$ and $\varphi_{0}$ satisfy Assumption 1.1 and let $\left(\psi_{t}, \varphi_{t}\right)$ be the solution of (1-8), (1-9) with initial condition $\left(\psi_{0}, \varphi_{0}\right)$. Define

$$
\begin{aligned}
\gamma_{t}^{\text {particle }} & :=\operatorname{Tr}_{\mathcal{F}}\left|e^{-i \tilde{H}_{\alpha}^{F} t} \psi_{0} \otimes W\left(\alpha^{2} \varphi_{0}\right) \Omega\right\rangle\left\langle e^{-i \tilde{H}_{\alpha}^{F} t} \psi_{0} \otimes W\left(\alpha^{2} \varphi_{0}\right) \Omega\right|, \\
\gamma_{t}^{\text {field }} & :=\operatorname{Tr}_{\mathcal{L}^{2}\left(\mathbb{R}^{3}\right)}\left|e^{-i \tilde{H}_{\alpha}^{F} t} \psi_{0} \otimes W\left(\alpha^{2} \varphi_{0}\right) \Omega\right\rangle\left\langle e^{-i \widetilde{H}_{\alpha}^{F} t} \psi_{0} \otimes W\left(\alpha^{2} \varphi_{0}\right) \Omega\right| .
\end{aligned}
$$

Then, for all $\alpha \geq 1$ and all $t \in[-\alpha, \alpha]$,

$$
\begin{array}{r}
\left.\operatorname{Tr}_{\mathcal{L}^{2}\left(\mathbb{R}^{3}\right)}\left|\gamma_{t}^{\text {particle }}-\right| \psi_{t}\right\rangle\left\langle\psi_{t}|| \leq C \alpha^{-2}\left(1+t^{2}\right),\right. \\
\left.\operatorname{Tr}_{\mathcal{F}}\left|\gamma_{t}^{\text {field }}-\right| W\left(\alpha^{2} \varphi_{t}\right) \Omega\right\rangle\left\langle W\left(\alpha^{2} \varphi_{t}\right) \Omega|| \leq C \alpha^{-2}\left(1+t^{2}\right) .\right.
\end{array}
$$

Note that $\gamma_{t}^{\text {particle }}, \gamma_{t}^{\text {field }},\left|\psi_{t}\right\rangle\left\langle\psi_{t}\right|$ and $\left|W\left(\alpha^{2} \varphi_{t}\right) \Omega\right\rangle\left\langle W\left(\alpha^{2} \varphi_{t}\right) \Omega\right|$ all have trace norm equal to one (in fact, they are nonnegative operators with trace one) and therefore Theorem 1.2 gives a nontrivial approximation up to times $t=o(\alpha)$. Already the approximation up to times of order one is significant since this is the time scale on which $\psi_{t}$ changes. It is a bonus that the same approximation is in fact valid for much longer times.

We emphasize that the Landau-Pekar approximation to the Fröhlich dynamics depends on $\alpha$ (through (1-9)). As we will explain in Section 1C, without allowing for an $\alpha$-dependence one cannot approximate $\gamma_{t}^{\text {particle }}$ with accuracy $\alpha^{-2}$ for times of order one.

We next present a more precise result which comes at the expense of a more complicated formulation. We approximate the state $e^{-i \widetilde{H}_{\alpha}^{F} t} \psi_{0} \otimes W\left(\alpha^{2} \varphi_{0}\right) \Omega$ itself in $\mathcal{L}^{2}\left(\mathbb{R}^{3}\right) \otimes \mathcal{F}$, and not only its reduced density matrices. However, it turns out that up to the desired order $\alpha^{-2}$ this is not possible in terms of simple product states. Instead, we need to include an explicit nonproduct state of order $\alpha^{-1}$ which takes correlations between the particle and the field into account. The key observation is that this term 
satisfies an almost orthogonality condition, so that it does not contribute to the reduced density matrices to order $\alpha^{-1}$. For the statement we need the real scalar function $\omega$ defined as

$$
\omega(t):=\alpha^{2} \operatorname{Im}\left(\varphi_{t}, \partial_{t} \varphi_{t}\right)+\left\|\varphi_{t}\right\|_{\mathcal{L}^{2}\left(\mathbb{R}^{3}\right)}^{2} .
$$

It will follow from Lemma 2.1 below that this function is uniformly bounded in $t \in \mathbb{R}$.

The following is our main result.

Theorem 1.3. Assume $\psi_{0}$ and $\varphi_{0}$ satisfy Assumption 1.1 and let $\left(\psi_{t}, \varphi_{t}\right)$ be the solution of (1-8), (1-9) with initial condition $\left(\psi_{0}, \varphi_{0}\right)$. Then there is a decomposition

$$
e^{-i \tilde{H}_{\alpha}^{F} t} \psi_{0} \otimes W\left(\alpha^{2} \varphi_{0}\right) \Omega=e^{-i \int_{0}^{t} \omega(s) d s} \psi_{t} \otimes W\left(\alpha^{2} \varphi_{t}\right) \Omega+R(t)
$$

and a constant $C>0$ such that for all $\alpha \geq 1$ and all $t \in[-\alpha, \alpha]$,

$$
\begin{gathered}
\left\|\left\langle\Omega, W^{*}\left(\alpha^{2} \varphi_{t}\right) R(t)\right\rangle_{\mathcal{F}}\right\|_{\mathcal{L}^{2}\left(\mathbb{R}^{3}\right)} \leq C \alpha^{-2}|t|(1+|t|), \\
\|\left\langle\psi_{t}, W^{*}\left(\alpha^{2} \varphi_{t}\right) R(t)\right\rangle_{\mathcal{L}^{2}\left(\mathbb{R}^{3}\right) \|_{\mathcal{F}}} \leq C \alpha^{-2}|t|(1+|t|)
\end{gathered}
$$

and

$$
\|R(t)\|_{\mathcal{L}^{2}\left(\mathbb{R}^{3}\right) \otimes \mathcal{F}} \leq C \alpha^{-1}(1+|t|) .
$$

More precisely, (1-13) holds with $R(t)=R_{1}(t)+R_{2}(t)$ and with the bounds

$$
\begin{gathered}
\left\|\left\langle\Omega, W^{*}\left(\alpha^{2} \varphi_{t}\right) R_{1}(t)\right\rangle_{\mathcal{F}}\right\|_{\mathcal{L}^{2}\left(\mathbb{R}^{3}\right)} \leq C \alpha^{-2} t^{2}, \\
\left\|\left\langle\psi_{t}, W^{*}\left(\alpha^{2} \varphi_{t}\right) R_{1}(t)\right\rangle_{\mathcal{L}^{2}\left(\mathbb{R}^{3}\right)}\right\|_{\mathcal{F}} \leq C \alpha^{-2} t^{2}
\end{gathered}
$$

and

$$
\left\|R_{2}(t)\right\|_{\mathcal{L}^{2}\left(\mathbb{R}^{3}\right) \otimes \mathcal{F}} \leq C \alpha^{-2}|t|(1+|t|), \quad\left\|R_{1}(t)\right\|_{\mathcal{L}^{2}\left(\mathbb{R}^{3}\right) \otimes \mathcal{F}} \leq C \alpha^{-1}(1+|t|) .
$$

Similarly as before, we note that for $t=o(\alpha)$ the term $R(t)$ is of lower order than the main term $e^{-i \int_{0}^{t} \omega(s) d s} \psi_{t} \otimes W\left(\alpha^{2} \varphi_{t}\right) \Omega$, which has constant norm equal to one.

The message of Theorem 1.3 is that, while $R(t)$ is in general not of order $\alpha^{-2}$ (for times of order one), it can be split into a piece which is, namely $R_{2}(t)$, and a piece which satisfies almost orthogonality conditions, so that it does not contribute to the reduced particle or field density matrices at order $\alpha^{-1}$ either. The term $R_{1}(t)$ is given explicitly in (2-16) below.

Theorem 1.3 implies Theorem 1.2 by a simple abstract argument, which we explain in Appendix D. In the following we concentrate on proving Theorem 1.3.

In Section 1C we compare Theorem 1.3 with a similar approximation in [Frank and Schlein 2014] where $\varphi_{t}$ is independent of $t$. In Lemma 1.4 we show that this simpler approximation does not yield the same accuracy in terms of powers of $\alpha^{-1}$ as Theorem 1.3. In this sense Theorem 1.3 derives the Landau-Pekar dynamics from the Fröhlich dynamics and answers an open question in [Frank and Schlein 2014].

While it is necessary to take the time dependence of $\varphi_{t}$ into account, this dependence is still weak for times of order $\alpha$ as considered in our theorems. The field $\varphi_{t}$ changes by order one only on times of order $\alpha^{2}$, and it would be desirable to extend Theorems 1.2 and 1.3 to this time scale, at least for a certain class of initial conditions. This remains an open problem. 
The key point in Theorem 1.3 and novel aspect of this work are the almost orthogonality relations (1-14) and (1-15). As we will see in Section 1C, they will be crucial for deriving Theorem 1.2. Inequality (1-16) is not sufficient for this purpose. Let us discuss the motivation behind the almost orthogonality relations in more detail. We introduce the function

$$
\tilde{\psi}_{t}:=e^{-i \int_{0}^{t} \omega(s) d s} \psi_{t}
$$

and consider the problem of approximating $e^{-i \tilde{H}_{\alpha}^{F} t} \psi_{0} \otimes W\left(\alpha^{2} \varphi_{0}\right) \Omega$ by a function of the form $\tilde{\psi}_{t} \otimes$ $W\left(\alpha^{2} \varphi_{t}\right) \Omega$. (We do not assume at this point that $\tilde{\psi}_{t}$ and $\varphi_{t}$ satisfy an equation.) Since $W\left(\alpha^{2} \varphi_{t}\right)$ is unitary, this is the same as the problem of choosing $\tilde{\psi}_{t}$ and $\varphi_{t}$ so as to minimize the norm of the vector

$$
W^{*}\left(\alpha^{2} \varphi_{t}\right) e^{-i \tilde{H}_{\alpha}^{F} t} \psi_{0} \otimes W\left(\alpha^{2} \varphi_{0}\right) \Omega-\tilde{\psi}_{t} \otimes \Omega
$$

Clearly, for given $\psi_{0}, \varphi_{0}$ and $\varphi_{t}$, the optimal choice for $\tilde{\psi}_{t}$ is

$$
\tilde{\psi}_{t}=\left\langle\Omega, W^{*}\left(\alpha^{2} \varphi_{t}\right) e^{-i \widetilde{H}_{\alpha}^{F} t} \psi_{0} \otimes W\left(\alpha^{2} \varphi_{0}\right) \Omega\right\rangle_{\mathcal{F}}
$$

In order to determine $\varphi_{t}$ we only solve the simpler problem of minimizing the norm of the projection of (1-21) onto the subspace $\operatorname{span}\left\{\tilde{\psi}_{t}\right\} \otimes \mathcal{F}$. This norm could be made zero if we could achieve

$$
\Omega=\left\langle\tilde{\psi}_{t}, W^{*}\left(\alpha^{2} \varphi_{t}\right) e^{-i \tilde{H}_{\alpha}^{F} t} \psi_{0} \otimes W\left(\alpha^{2} \varphi_{0}\right) \Omega\right\rangle_{\mathcal{L}^{2}}
$$

While it may not be possible to have exact equalities in (1-22) and (1-23), we will see that the LandauPekar equations yield almost equalities. In fact, the almost orthogonality relations (1-14) and (1-15) in our main theorem state exactly that:

$$
\begin{aligned}
& \tilde{\psi}_{t}-\left\langle\Omega, W^{*}\left(\alpha^{2} \varphi_{t}\right) e^{-i \tilde{H}_{\alpha}^{F} t} \psi_{0} \otimes W\left(\alpha^{2} \varphi_{0}\right) \Omega\right\rangle_{\mathcal{F}}=O_{\mathcal{L}^{2}}\left(\alpha^{-2}|t|(1+|t|)\right), \\
& \Omega-\left\langle\tilde{\psi}_{t}, W^{*}\left(\alpha^{2} \varphi_{t}\right) e^{-i \tilde{H}_{\alpha}^{F} t} \psi_{0} \otimes W\left(\alpha^{2} \varphi_{0}\right) \Omega\right\rangle_{\mathcal{L}^{2}}=O_{\mathcal{F}}\left(\alpha^{-2}|t|(1+|t|)\right) .
\end{aligned}
$$

1C. Comparison with earlier results. The problem of approximating the Fröhlich dynamics of a polaron was studied before in [Frank and Schlein 2014]. There a different and simpler effective equation is proposed in which only the particles move and the phonon field remains constant. In this subsection we show that Theorem 1.2 is not valid for these effective dynamics from [Frank and Schlein 2014], in the sense that the reduced phonon density matrix cannot be approximated to within an error $\alpha^{-2}$ for times of order one. The fact that our Theorem 1.2 achieves an approximation at this accuracy is because the phonon motion is taken into account in the Landau-Pekar equations. Technically this is reflected in the orthogonality conditions (1-14) and (1-15).

To be more specific we recall that in [Frank and Schlein 2014] it was shown that

$$
\left\|e^{-i \widetilde{H}_{\alpha}^{F} t} \psi_{0} \otimes W\left(\alpha^{2} \varphi_{0}\right) \Omega-e^{-i\left\|\varphi_{0}\right\|_{2}^{2} t} \zeta_{t} \otimes W\left(\alpha^{2} \varphi_{0}\right) \Omega\right\|_{\mathcal{L}^{2} \otimes \mathcal{F}} \leq C \alpha^{-1}\left(e^{C|t|}-1\right)^{\frac{1}{2}}
$$

where $\zeta_{t}$ denotes the solution of the linear equation

$$
i \partial_{t} \zeta_{t}(x)=\left[-\Delta+\int_{\mathbb{R}^{3}}\left[e^{-i k x} \varphi_{0}(k)+e^{i k \cdot x} \overline{\varphi_{0}(k)}\right] \frac{d k}{|k|}\right] \zeta_{t}(x)
$$


with initial condition $\psi_{0}$. We stress again that in this approximation, $\varphi_{0}$ does not evolve in time. An anonymous referee, to whom we are most grateful, has explained to us that the method of [Frank and Schlein 2014] actually leads to the bound

$$
\left\|e^{-i \widetilde{H}_{\alpha}^{F} t} \psi_{0} \otimes W\left(\alpha^{2} \varphi_{0}\right) \Omega-e^{-i\left\|\varphi_{0}\right\|_{2}^{2} t} \zeta_{t} \otimes W\left(\alpha^{2} \varphi_{0}\right) \Omega\right\|_{\mathcal{L}^{2} \otimes \mathcal{F}} \leq C\left(e^{C|t| / \alpha}-1\right)^{\frac{1}{2}},
$$

which provides an approximation even up to times of order $o(\alpha)$. With his/her kind permission we reproduce the argument in Appendix E.

As an aside we note that we recover a similar bound as a simple consequence of Theorem 1.3. (In fact, our new bound is better by a power of $\alpha^{-\frac{1}{2}}$ for times $t \gg 1$.) Namely, (1-16) says that

$$
\left\|e^{-i \widetilde{H}_{\alpha}^{F} t} \psi_{0} \otimes W\left(\alpha^{2} \varphi_{0}\right) \Omega-e^{-i \int_{0}^{t} \omega(s) d s} \psi_{t} \otimes W\left(\alpha^{2} \varphi_{t}\right) \Omega\right\|_{\mathcal{L}^{2} \otimes \mathcal{F}} \leq C \alpha^{-1}(1+|t|) .
$$

(In [Frank and Schlein 2014] weaker regularity and decay assumptions are imposed on $\psi_{0}$ and $\varphi_{0}$, but we emphasize that (1-16) is also valid under weaker assumptions than those in Assumption 1.1. In fact, the latter assumption is needed to bound $R_{2}(t)$, whereas for (1-16) one can avoid the use of Duhamel's principle in Proposition 2.3.)

For the reduced density matrices, inequalities (1-26) and (1-27) give, using (D-1) and possibly changing the value of $C$,

$$
\begin{array}{r}
\left.\operatorname{Tr}_{\mathcal{L}^{2}}\left|\gamma_{t}^{\text {particle }}-\right| \zeta_{t}\right\rangle\left\langle\zeta_{t}|| \leq C \min \left\{\alpha^{-1}\left(e^{C|t|}-1\right)^{\frac{1}{2}},\left(e^{C|t| / \alpha}-1\right)^{\frac{1}{2}}\right\},\right. \\
\operatorname{Tr}_{\left.\mathcal{L}^{2}\left|\gamma_{t}^{\text {field }}-\right| W\left(\alpha^{2} \varphi_{0}\right) \Omega\right\rangle\left\langle W\left(\alpha^{2} \varphi_{0}\right) \Omega||\right.} \leq C \min \left\{\alpha^{-1}\left(e^{C|t|}-1\right)^{\frac{1}{2}},\left(e^{C|t| / \alpha}-1\right)^{\frac{1}{2}}\right\} .
\end{array}
$$

These bounds behave like $\alpha^{-1}$ for times of order one.

The next result shows that in this approximation of $\gamma_{t}^{\text {field }}$ by a time-independent $\varphi_{0}$ the order $\alpha^{-1}$ (for times of order one) cannot be improved in general.

Lemma 1.4. In addition to Assumption 1.1 suppose that $\varphi_{0} \not \equiv-\sigma_{\psi_{0}}$ in the notation (2-2). Then there are $\varepsilon>0, C>0$ and $c>0$ such that for all $|t| \in\left[C \alpha^{-1}, \varepsilon\right]$ and all $\alpha \geq C / \varepsilon$,

$$
\left.\operatorname{Tr}_{\mathcal{F}}\left|\gamma_{t}^{\text {field }}-\right| W\left(\alpha^{2} \varphi_{0}\right) \Omega\right\rangle\left\langle W\left(\alpha^{2} \varphi_{0}\right) \Omega|| \geq c \alpha^{-1}|t|\right.
$$

This lemma should be contrasted with Theorem 1.2, which says that the time-dependent approximation $\left|W\left(\alpha^{2} \varphi_{t}\right) \Omega\right\rangle\left\langle W\left(\alpha^{2} \varphi_{t}\right) \Omega\right|$ is correct to order $\alpha^{-2}$ (for times of order one). This argument shows the importance of the orthogonality conditions (1-14) and (1-15). Indeed, if we would only use (1-16), we would arrive at (1-28) and this would again only give an approximation to order $\alpha^{-1}$ (for times of order one).

Since Theorem 1.2 is a consequence of Theorem 1.3 and since we showed that one cannot replace $\varphi_{t}$ by $\varphi_{0}$ in Theorem 1.2, the same applies also to Theorem 1.3.

Let us consider our problem from a wider perspective. We have a composite quantum system $\mathcal{H}_{1} \otimes \mathcal{H}_{2}$ and a Hamiltonian which couples the two subsystems. Each system has an effective "Planck constant" and the characteristic feature of the problem is that the Planck constant of one system goes to zero, whereas that of the other system remains fixed. Thus, one of the systems becomes classical, whereas the other one 
remains quantum-mechanical, and Ginibre, Nironi and Velo [Ginibre et al. 2006] used the term "partially classical limit" in a closely related context. (For us, the "Planck constant" of the phonons is $\alpha^{-2}$, as can be seen from the commutation relations, whereas that of the electron is of order one.) A prime example of such a problem is the Born-Oppenheimer approximation, where the inverse square root of the nuclear mass plays the role of the small Planck constant.

Here, however, we consider the case where $\mathcal{H}_{1} \otimes \mathcal{H}_{2}$ has infinitely many degrees of freedom. As is well known, our Hamiltonian is the Wick quantization of an energy functional on an infinite-dimensional phase space and the notion of "Planck constant" has a well-defined meaning through the commutation relations of the fields. (We emphasize that in our problem we can imagine that we have also a field $\Psi$ for the electrons, but that we only consider the sector of a single electron.)

Although there is an enormous literature concerning the classical limit, starting with Hepp's work [1974], and although we believe that the question of a partially classical limit is a very natural one which appears in many models, we are only aware of the single work [Ginibre et al. 2006] prior to [Frank and Schlein 2014] on this question, and it studies fluctuation dynamics. Closer to our focus here are the works [Falconi 2013; Ammari and Falconi 2014] about the Nelson model with a cut-off where, however, a classical limit on both systems is taken. On the level of results, one obtains equations similar to the Landau-Pekar equations (without the factor $\alpha^{2}$ in (1-9)), but the proofs are completely different, as [Ammari and Falconi 2014] relies on the Wigner measure approach from [Ammari and Nier 2008; 2009].

The polaron model, in contrast to the Nelson model, does not require a cut-off, although this is not obvious since the operator $\int e^{i k \cdot x} b_{k}|k|^{-1} d k$ and its adjoint are not bounded relative to the number operator. Lieb and Yamazaki [1958] devised a method to deal with this problem in the stationary case, but it is not clear to us how to apply their argument in a dynamical setting and we consider our solution of this problem as a technical novelty in this paper. Our methods apply equally well to a partially classical limit in the cut-off Nelson model and, in fact, the proofs in that case would be considerably shorter.

1D. An equivalent form of the Landau-Pekar equations. Often the Landau-Pekar equations are stated in the form

$$
\begin{aligned}
i \partial_{t} \psi_{t} & =\left(-\Delta+|x|^{-1} * P_{t}\right) \psi_{t}, \\
\alpha^{4} \partial_{t}^{2} P_{t} & =-P_{t}-(2 \pi)^{2}\left|\psi_{t}\right|^{2}
\end{aligned}
$$

for a real-valued polarization field $P_{t}$; see, e.g., [Landau and Pekar 1948; Devreese and Alexandrov 2009]. Let us show that this pair of equations is equivalent to the pair of equations that we discussed so far. In fact, assume that $\psi_{t}$ and $\varphi_{t}$ solve (1-8) and (1-9) and define

$$
P_{t}(x):=(2 \pi)^{-1} \operatorname{Re} \int_{\mathbb{R}^{3}}|k| \varphi_{t}(k) e^{-i k \cdot x} d k,
$$

as well as the auxiliary function

$$
Q_{t}(x):=(2 \pi)^{-1} \operatorname{Im} \int_{\mathbb{R}^{3}}|k| \varphi_{t}(k) e^{-i k \cdot x} d k .
$$


If we multiply (1-9) by $|k|$ and integrate with respect to $e^{-i k \cdot x}$, we obtain

$$
i \alpha^{2} \partial_{t}\left(P_{t}+i Q_{t}\right)=P_{t}+i Q_{t}+(2 \pi)^{2}\left|\psi_{t}\right|^{2} .
$$

Since $P_{t}$ and $Q_{t}$ are real, this equation is equivalent to the pair of equations

$$
\alpha^{2} \partial_{t} P_{t}=Q_{t}, \quad \alpha^{2} \partial_{t} Q_{t}=-P_{t}-(2 \pi)^{2}\left|\psi_{t}\right|^{2} .
$$

Here we can eliminate $Q_{t}$ by differentiating the first equation and arrive at (1-30).

Moreover, the inversion formula

$$
\varphi_{t}(k)=(2 \pi)^{-2}|k|^{-1} \int_{\mathbb{R}^{3}}\left(P_{t}+i Q_{t}\right) e^{i k \cdot x} d x
$$

implies

$$
\int_{\mathbb{R}^{3}}\left(e^{-i k \cdot x} \varphi_{t}(k)+e^{i k \cdot x} \overline{\varphi_{t}(k)}\right) \frac{d k}{|k|}=|x|^{-1} * P_{t},
$$

which yields (1-29).

\section{Outline of the proof}

2A. Well-posedness of the Landau-Pekar equations. We begin by discussing the well-posedness of the equations for $\psi_{t}$ and $\varphi_{t}$ in (1-8) and (1-9). We use the following abbreviations for the coupling terms in these equations,

$$
V_{\varphi}(x):=\int_{\mathbb{R}^{3}}\left[e^{-i k \cdot x} \varphi(k)+e^{i k \cdot x} \overline{\varphi(k)}\right] \frac{d k}{|k|}
$$

and

$$
\sigma_{\psi}(k):=|k|^{-1} \int_{\mathbb{R}^{3}}|\psi(x)|^{2} e^{i k \cdot x} d x .
$$

The following lemma, which is proved in Appendix C, states global well-posedness in the energy space $\mathcal{H}^{1}\left(\mathbb{R}^{3}\right) \times \mathcal{L}^{2}\left(\mathbb{R}^{3}\right)$.

Lemma 2.1. For any $\left(\psi_{0}, \varphi_{0}\right) \in \mathcal{H}^{1}\left(\mathbb{R}^{3}\right) \times \mathcal{L}^{2}\left(\mathbb{R}^{3}\right)$ there is a unique global solution $\left(\psi_{t}, \varphi_{t}\right)$ of (1-8), (1-9). One has the conservation laws

$$
\left\|\psi_{t}\right\|_{\mathcal{L}^{2}}=\left\|\psi_{0}\right\|_{\mathcal{L}^{2}} \quad \text { and } \quad \mathcal{E}\left(\psi_{t}, \varphi_{t}\right)=\mathcal{E}\left(\psi_{0}, \varphi_{0}\right) \quad \text { for all } t \in \mathbb{R} .
$$

Moreover, for all $\alpha>0$ and all $t \in \mathbb{R}$,

$$
\left\|\psi_{t}\right\|_{\mathcal{H}^{1}} \lesssim 1, \quad\left\|\varphi_{t}\right\|_{\mathcal{L}^{2}} \lesssim 1
$$

and

$$
\left\|\partial_{t} \varphi_{t}\right\|_{\mathcal{L}^{2}} \lesssim \alpha^{-2}, \quad\left\|\varphi_{t}-\varphi_{s}\right\|_{\mathcal{L}^{2}} \lesssim \alpha^{-2}|t-s|, \quad\left\|\sigma_{\psi_{t}}\right\|_{\mathcal{L}^{2}} \lesssim 1 .
$$

In the proof of our main result we need to go beyond the energy space $\mathcal{H}^{1}\left(\mathbb{R}^{3}\right) \times \mathcal{L}^{2}\left(\mathbb{R}^{3}\right)$. The following proposition states that if the initial conditions have more regularity and decay then, at least for a certain 
(long) time interval, we have bounds on the solution in the corresponding spaces. We will also need some bounds on the auxiliary functions $g_{s, t}: \mathbb{R}^{3} \rightarrow \mathbb{C}$ defined by

$$
g_{s, t}(x):=\int_{\mathbb{R}^{3}}\left[\overline{\varphi_{t}(k)}-\overline{\varphi_{s}(k)}\right] e^{i k \cdot x} \frac{d k}{|k|}
$$

and $g_{s}: \mathbb{R}^{3} \rightarrow \mathbb{C}$ defined by

$$
g_{s}(x):=-\partial_{s} g_{s, t}(x)=\int_{\mathbb{R}^{3}} e^{i k \cdot x} \overline{\partial_{s} \varphi_{s}(k)} \frac{d k}{|k|} .
$$

The following proposition will also be proved in Appendix C.

Proposition 2.2. Let $\tau>0$. If $\left(\psi_{0}, \varphi_{0}\right)$ satisfies Assumption 1.1, then for all $\alpha>0$ and for all $t, s \in$ $\left[-\tau \alpha^{2}, \tau \alpha^{2}\right]$ we have

Moreover,

$$
\left\|\psi_{t}\right\|_{\mathcal{H}^{4}} \lesssim_{\tau} 1, \quad\left\|\varphi_{t}\right\|_{\mathcal{L}_{(3)}^{2}} \lesssim_{\tau} 1
$$

$$
\left\|\partial_{t} \psi_{t}\right\|_{\mathcal{H}^{2}} \lesssim_{\tau} 1, \quad\left\|\partial_{t} \sigma_{\psi_{t}}\right\|_{\mathcal{L}^{2}} \lesssim_{\tau} 1
$$

and

$$
\left\|g_{s, t}\right\|_{\infty} \lesssim_{\tau} \alpha^{-2}|t-s|, \quad\left\|g_{s}\right\|_{\infty} \lesssim \alpha^{-2} .
$$

2B. Decomposition of the solution. We now decompose the solution $e^{-i \widetilde{H}_{\alpha}^{F} t} \psi_{0} \otimes W\left(\alpha^{2} \varphi_{0}\right) \Omega$ as claimed in Theorem 1.3. In order to state this, we need to introduce some notations.

It will be convenient to work with the function $\tilde{\psi}_{t}$ from (1-20). Clearly, the bounds from Lemma 2.1 and Proposition 2.2 hold for $\tilde{\psi}_{t}$ as well. (For the bounds on $\partial_{t} \tilde{\psi}_{t}$ we use the fact that $|\omega(t)| \lesssim 1$ by Lemma 2.1.) Moreover, we note that $\tilde{\psi}_{t}$ and $\varphi_{t}$ satisfy the modified equations

$$
\begin{aligned}
i \partial_{t} \tilde{\psi}_{t}(x) & =\left[-\Delta+\int_{\mathbb{R}^{3}}\left[e^{-i k \cdot x} \varphi_{t}(k)+e^{i k \cdot x} \bar{\varphi}_{t}(k)\right] \frac{d k}{|k|}+\omega(t)\right] \tilde{\psi}_{t}(x), \\
i \alpha^{2} \partial_{t} \varphi_{t}(k) & =\varphi_{t}(k)+|k|^{-1} \int_{\mathbb{R}^{3}}\left|\tilde{\psi}_{t}(x)\right|^{2} e^{i k \cdot x} d x .
\end{aligned}
$$

Next, we define for $\psi \in \mathcal{L}^{2}\left(\mathbb{R}^{3}\right)$ with $\|\psi\|=1$ the orthogonal projections in $\mathcal{L}^{2}\left(\mathbb{R}^{3}\right)$,

$$
P_{\psi}:=|\psi\rangle\left\langle\psi\left|, \quad P_{\psi}^{\perp}:=1-P_{\psi}=1-\right| \psi\right\rangle\langle\psi| .
$$

The effective Schrödinger operator $H_{\varphi}$ in $\mathcal{L}^{2}\left(\mathbb{R}^{3}\right)$ is defined by

$$
H_{\varphi}:=-\Delta+V_{\varphi}+\int_{\mathbb{R}^{3}}|\varphi(k)|^{2} d k
$$

with $V_{\varphi}$ from (2-1). Moreover, let us introduce the operator

$$
\tilde{H}_{\varphi}:=W^{*}\left(\alpha^{2} \varphi\right) \widetilde{H}_{\alpha}^{F} W\left(\alpha^{2} \varphi\right)
$$

in $\mathcal{L}^{2}\left(\mathbb{R}^{3}\right) \otimes \mathcal{F}$. Using the commutation relations (see Lemma A.1) we find that

$$
\tilde{H}_{\varphi}=H_{\varphi}+\int_{\mathbb{R}^{3}}\left[e^{i k \cdot x} b_{k}^{*}+e^{-i k \cdot x} b_{k}\right] \frac{d k}{|k|}+\int_{\mathbb{R}^{3}}\left[\varphi(k) b_{k}^{*}+\bar{\varphi}(k) b_{k}\right] d k+\int_{\mathbb{R}^{3}} b_{k}^{*} b_{k} d k .
$$


Finally, we introduce the vector

and define

$$
F_{t, s}:=P \frac{\perp}{\tilde{\psi}_{s}} \int_{\mathbb{R}^{3}}\left(e^{i k \cdot x} W^{*}\left(\alpha^{2} \varphi_{t}\right) W\left(\alpha^{2} \varphi_{s}\right) b_{k}^{*} \tilde{\psi}_{s} \otimes \Omega\right) \frac{d k}{|k|}
$$

and

$$
D_{0}:=\int_{0}^{t} e^{i H_{\varphi_{t}} s} F_{t, s} d s
$$

$$
\begin{aligned}
D_{1} & :=\int_{0}^{t} \int_{0}^{t-s} \int_{\mathbb{R}^{3}}\left(e^{i \widetilde{H}_{\varphi_{t}}\left(s+s_{1}\right)} e^{i k \cdot x} b_{k}^{*} e^{-i H_{\varphi_{t}} s_{1}} F_{t, s}\right) \frac{d k}{|k|} d s_{1} d s, \\
D_{2} & :=\int_{0}^{t} \int_{0}^{t-s} \int_{\mathbb{R}^{3}}\left(e^{i \widetilde{H}_{\varphi_{t}}\left(s+s_{1}\right)} e^{-i k \cdot x} b_{k} e^{-i H_{\varphi_{t}} s_{1}} F_{t, s}\right) \frac{d k}{|k|} d s_{1} d s, \\
D_{3} & :=\int_{0}^{t} \int_{0}^{t-s} \int_{\mathbb{R}^{3}}\left(e^{i \widetilde{H}_{\varphi_{t}}\left(s+s_{1}\right)} \varphi_{t}(k) b_{k}^{*} e^{-i H_{\varphi_{t}} s_{1}} F_{t, s}\right) d k d s_{1} d s, \\
D_{4} & :=\int_{0}^{t} \int_{0}^{t-s} \int_{\mathbb{R}^{3}}\left(e^{i \widetilde{H}_{\varphi_{t}}\left(s+s_{1}\right)} \overline{\varphi_{t}(k)} b_{k} e^{-i H_{\varphi_{t}} s_{1}} F_{t, s}\right) d k d s_{1} d s, \\
D_{5} & :=\int_{0}^{t} \int_{0}^{t-s} \int_{\mathbb{R}^{3}}\left(e^{i \widetilde{H}_{\varphi_{t}}\left(s+s_{1}\right)} b_{k}^{*} b_{k} e^{-i H_{\varphi_{t}} s_{1}} F_{t, s}\right) d k d s_{1} d s .
\end{aligned}
$$

While these definitions might seem formal, we will show in Theorem 2.5 that each of $D_{0}, \ldots, D_{5}$ belongs to $\mathcal{L}^{2}\left(\mathbb{R}^{3}\right) \otimes \mathcal{F}$.

With these notations, the promised representation formula for the solution looks as follows.

Proposition 2.3. Assume that $\left(\tilde{\psi}_{t}, \varphi_{t}\right)$ satisfy (2-10), (2-11) with initial conditions $\left(\psi_{0}, \varphi_{0}\right)$ where $\left\|\psi_{0}\right\|^{2}=1$. Then for any $t \in \mathbb{R}$ one has the decomposition

$$
e^{-i \tilde{H}_{\alpha}^{F} t} \psi_{0} \otimes W\left(\alpha^{2} \varphi_{0}\right) \Omega=\tilde{\psi}_{t} \otimes W\left(\alpha^{2} \varphi_{t}\right) \Omega+R_{1}(t)+R_{2}(t)
$$

with

$$
R_{1}(t):=-i W\left(\alpha^{2} \varphi_{t}\right) e^{-i H_{\varphi_{t}} t} D_{0}
$$

and

$$
R_{2}(t):=-W\left(\alpha^{2} \varphi_{t}\right) e^{-i \widetilde{H}_{\varphi_{t}} t}\left(D_{1}+D_{2}+D_{3}+D_{4}+D_{5}\right) .
$$

Clearly, in terms of the original function $\psi_{t}$, the term $R_{1}$ is explicitly given by $R_{1}(t)=$

$$
-i W\left(\alpha^{2} \varphi_{t}\right) \int_{0}^{t}\left[e^{-i H_{\varphi_{t}}(t-s)-i \int_{0}^{s} \omega\left(s_{1}\right) d s_{1}} P_{\psi_{s}}^{\perp} \int_{\mathbb{R}^{3}}\left(e^{i k \cdot x} W^{*}\left(\alpha^{2} \varphi_{t}\right) W\left(\alpha^{2} \varphi_{s}\right) b_{k}^{*} \psi_{s} \otimes \Omega\right) \frac{d k}{|k|}\right] d s .
$$

The proof of Proposition 2.3 makes use of equations (2-10), (2-11) for $\left(\tilde{\psi}_{t}, \varphi_{t}\right)$ as well as the Duhamel formula. We single out the use of the equations in the following lemma.

Lemma 2.4. Assume that $\left(\tilde{\psi}_{t}, \varphi_{t}\right)$ satisfy (2-10), (2-11) with initial conditions $\left(\psi_{0}, \varphi_{0}\right)$ where $\left\|\psi_{0}\right\|^{2}=1$. Then for any $t \in \mathbb{R}$ one has

$$
e^{-i \widetilde{H}_{\alpha}^{F} t} \psi_{0} \otimes W\left(\alpha^{2} \varphi_{0}\right) \Omega=\tilde{\psi}_{t} \otimes W\left(\alpha^{2} \varphi_{t}\right) \Omega-i \int_{0}^{t} e^{-i \widetilde{H}_{\alpha}^{F}(t-s)} W\left(\alpha^{2} \varphi_{t}\right) F_{t, s} d s .
$$


Proof of Lemma 2.4. Applying the operator $e^{i \widetilde{H}_{\alpha}^{F} t}$ to both sides of (2-17) we see that we need to prove

$$
\psi_{0} \otimes W\left(\alpha^{2} \varphi_{0}\right) \Omega=e^{i \widetilde{H}_{\alpha}^{F} t} \tilde{\psi}_{t} \otimes W\left(\alpha^{2} \varphi_{t}\right) \Omega-i \int_{0}^{t} e^{i \widetilde{H}_{\alpha}^{F} s} W\left(\alpha^{2} \varphi_{t}\right) F_{t, s} d s
$$

This is clearly true at $t=0$ and therefore we only need to show that the time derivatives of both sides coincide for all $t$; that is, in view of definition (2-15) of $F_{t, s}$,

$$
\begin{aligned}
0=e^{i \tilde{H}_{\alpha}^{F} t}\left[i \tilde{H}_{\alpha}^{F} \tilde{\psi}_{t} \otimes W\left(\alpha^{2} \varphi_{t}\right) \Omega+\partial_{t} \tilde{\psi}_{t} \otimes W\left(\alpha^{2} \varphi_{t}\right) \Omega+\right. & \tilde{\psi}_{t} \otimes \partial_{t} W\left(\alpha^{2} \varphi_{t}\right) \Omega \\
& \left.-i W\left(\alpha^{2} \varphi_{t}\right) P \tilde{\psi}_{t} \int_{\mathbb{R}^{3}}\left(e^{i k \cdot x} b_{k}^{*} \tilde{\psi}_{t} \otimes \Omega\right) \frac{d k}{|k|}\right] .
\end{aligned}
$$

This is, of course, the same as

$i \tilde{H}_{\alpha}^{F} \tilde{\psi}_{t} \otimes W\left(\alpha^{2} \varphi_{t}\right) \Omega+\partial_{t} \tilde{\psi}_{t} \otimes W\left(\alpha^{2} \varphi_{t}\right) \Omega+\tilde{\psi}_{t} \otimes \partial_{t} W\left(\alpha^{2} \varphi_{t}\right) \Omega$

$$
=i W\left(\alpha^{2} \varphi_{t}\right) P_{\tilde{\psi}_{t}}^{\perp} \int_{\mathbb{R}^{3}}\left(e^{i k \cdot x} b_{k}^{*} \tilde{\psi}_{t} \otimes \Omega\right) \frac{d k}{|k|},
$$

which is what we are going to show now.

We begin by rewriting the first term on the left side. Using (2-13) and (2-14) we obtain

$$
i \widetilde{H}_{\alpha}^{F} \tilde{\psi}_{t} \otimes W\left(\alpha^{2} \varphi_{t}\right) \Omega=i H_{\varphi_{t}} \tilde{\psi}_{t} \otimes W\left(\alpha^{2} \varphi_{t}\right) \Omega+\tilde{\psi}_{t} W\left(\alpha^{2} \varphi_{t}\right)\left[i b^{*}\left(\varphi_{t}\right)+i \int_{\mathbb{R}^{3}} e^{i k \cdot x} b_{k}^{*} \frac{d k}{|k|}\right] \Omega .
$$

In order to rewrite the third term on the left side of (2-18) we use the formula for $\partial_{t} W\left(\alpha^{2} \varphi_{t}\right)$ from (A-4) below and find

$$
\tilde{\psi}_{t} \otimes \partial_{t} W\left(\alpha^{2} \varphi_{t}\right) \Omega=i \alpha^{2}\left(\operatorname{Im}\left(\varphi_{t}, \partial_{t} \varphi_{t}\right)\right) \tilde{\psi}_{t} \otimes W\left(\alpha^{2} \varphi_{t}\right) \Omega+\alpha^{2} \tilde{\psi}_{t} \otimes W\left(\alpha^{2} \varphi_{t}\right) b^{*}\left(\partial_{t} \varphi_{t}\right) \Omega .
$$

Thus, recalling the definition of $\omega$ in (1-12), we have shown that

$$
\begin{aligned}
i \tilde{H}_{\alpha}^{F} \tilde{\psi}_{t} \otimes W\left(\alpha^{2} \varphi_{t}\right) \Omega+ & \partial_{t} \tilde{\psi}_{t} \otimes W\left(\alpha^{2} \varphi_{t}\right) \Omega+\tilde{\psi}_{t} \otimes \partial_{t} W\left(\alpha^{2} \varphi_{t}\right) \Omega \\
=[ & \left.\partial_{t}+i\left(-\Delta+V_{\varphi_{t}}+\omega(t)\right)\right] \tilde{\psi}_{t} \otimes W\left(\alpha^{2} \varphi_{t}\right) \Omega \\
& \quad+W\left(\alpha^{2} \varphi_{t}\right)\left[\alpha^{2} b^{*}\left(\partial_{t} \varphi_{t}\right)+i b^{*}\left(\varphi_{t}\right)+i \int_{\mathbb{R}^{3}} e^{i k \cdot x} b_{k}^{*} \frac{d k}{|k|}\right]\left(\tilde{\psi}_{t} \otimes \Omega\right) .
\end{aligned}
$$

At this point in the proof we use the equations for $\tilde{\psi}_{t}$ and $\varphi_{t}$. It follows from (2-10) that line (2-19) vanishes identically. For line (2-20) we use (2-11) to obtain

$$
\begin{aligned}
i \tilde{H}_{\alpha}^{F} \tilde{\psi}_{t} \otimes W\left(\alpha^{2} \varphi_{t}\right) \Omega+\partial_{t} \tilde{\psi}_{t} \otimes W\left(\alpha^{2} \varphi_{t}\right) \Omega+\tilde{\psi}_{t} \otimes \partial_{t} W\left(\alpha^{2} \varphi_{t}\right) \Omega \\
=i W\left(\alpha^{2} \varphi_{t}\right)\left[\int_{\mathbb{R}^{3}}\left(-\int_{\mathbb{R}^{3}}\left|\tilde{\psi}_{t}(y)\right|^{2} e^{i k \cdot y} d y+e^{i k \cdot x}\right) b_{k}^{*} \frac{d k}{|k|}\right]\left(\tilde{\psi}_{t} \otimes \Omega\right) \\
=i W\left(\alpha^{2} \varphi_{t}\right) P{ }_{\tilde{\psi}_{t}}^{\perp} \int_{\mathbb{R}^{3}}\left(e^{i k \cdot x} b_{k}^{*} \tilde{\psi}_{t} \otimes \Omega\right) \frac{d k}{|k|}
\end{aligned}
$$


Here we used the fact that $\left\|\tilde{\psi}_{t}\right\|=\left\|\psi_{0}\right\|=1$ by assumption and Lemma 2.1, and therefore

$$
P \underset{\tilde{\psi}_{t}}{\perp}=1-\left|\tilde{\psi}_{t}\right\rangle\left\langle\tilde{\psi}_{t}\right|
$$

Equation (2-21) proves (2-18) and completes the proof.

Having proved Lemma 2.4 we turn to the proof of Proposition 2.3.

Proof of Proposition 2.3. It follows from Lemma 2.4 and (2-13) that

$$
e^{-i \widetilde{H}_{\alpha}^{F} t} \psi_{0} \otimes W\left(\alpha^{2} \varphi_{0}\right) \Omega=\tilde{\psi}_{t} \otimes W\left(\alpha^{2} \varphi_{t}\right) \Omega-i W\left(\alpha^{2} \varphi_{t}\right) \int_{0}^{t} e^{-i \widetilde{H}_{\varphi_{t}}(t-s)} F_{t, s} d s .
$$

In the time integral on the right side we use Duhamel's principle and (2-14),

$$
\begin{aligned}
& e^{-i \widetilde{H}_{\varphi_{t}}(t-s)}=e^{-i H_{\varphi_{t}}(t-s)}-i \int_{0}^{t-s} e^{-i \widetilde{H}_{\varphi_{t}}\left(t-s-s_{1}\right)}\left(\int_{\mathbb{R}^{3}}\left[e^{i k \cdot x} b_{k}^{*}+e^{-i k \cdot x} b_{k}\right] \frac{d k}{|k|}+\int_{\mathbb{R}^{3}} b_{k}^{*} b_{k} d k\right. \\
& \left.+\int_{\mathbb{R}^{3}}\left[\varphi_{t}(k) b_{k}^{*}+\bar{\varphi}_{t}(k) b_{k}\right] d k\right) e^{-i H_{\varphi_{t}} s_{1}} d s_{1} .
\end{aligned}
$$

Proposition 2.3 now follows easily from the definition of $D_{0}, \ldots, D_{5}$.

2C. Reduction of the proof of the main result. In the remainder of this paper we will prove the following.

Theorem 2.5. Assume that $\psi_{0}$ and $\varphi_{0}$ satisfy Assumption 1.1, let $\left(\tilde{\psi}_{t}, \varphi_{t}\right)$ be the solution of (2-10), (2-11) with initial condition $\left(\psi_{0}, \varphi_{0}\right)$ and let $D_{0}, \ldots, D_{5}$ be as in Proposition 2.3. Then there is a constant $C>0$ such that for all $\alpha \geq 1$ and $t \in\left[0, \alpha^{2}\right]$,

$$
\begin{aligned}
\left\|D_{0}\right\|_{\mathcal{L}^{2} \otimes \mathcal{F}} & \leq C \alpha^{-1}(1+t), \\
\left\|D_{1}\right\|_{\mathcal{L}^{2} \otimes \mathcal{F}} & \leq C \alpha^{-2} t(1+t), \\
\left\|D_{2}\right\|_{\mathcal{L}^{2} \otimes \mathcal{F}} & \leq \alpha^{-2} t(1+t)\left(1+\alpha^{-1} t\right), \\
\left\|D_{3}\right\|_{\mathcal{L}^{2} \otimes \mathcal{F}} & \leq C \alpha^{-2} t(1+t)\left(1+\alpha^{-1} t\right), \\
\left\|D_{4}\right\|_{\mathcal{L}^{2} \otimes \mathcal{F}} & \leq C \alpha^{-2} t^{2}\left(1+\alpha^{-1} t\right), \\
\left\|D_{5}\right\|_{\mathcal{L}^{2} \otimes \mathcal{F}} & \leq C \alpha^{-3} t(1+t)\left(1+\alpha^{-2} t^{2}\right), \\
\left\|\left\langle\Omega, e^{-i H_{\varphi_{t}} t} D_{0}\right\rangle_{\mathcal{F}}\right\|_{\mathcal{L}^{2}\left(\mathbb{R}^{3}\right)} & \leq C \alpha^{-2} t^{2}, \\
\left\|\left\langle\tilde{\psi}_{t}, e^{-i H_{\varphi_{t}} t} D_{0}\right\rangle_{\mathcal{L}^{2}\left(\mathbb{R}^{3}\right)}\right\|_{\mathcal{F}} & \leq C \alpha^{-2} t^{2}\left(1+\alpha^{-2} t^{2}\right) .
\end{aligned}
$$

This theorem (and its analogue for $t \in\left[-\alpha^{2}, 0\right]$ ), together with the decomposition from Proposition 2.3 and the fact that the operators $W\left(\alpha^{2} \varphi_{t}\right), e^{-i H_{\varphi_{t}} t}$ and $e^{-i \widetilde{H}_{\varphi_{t}} t}$ are unitary, implies Theorem 1.3. In fact, (2-22) implies the second bound in (1-19), (2-23)-(2-27) imply the first bound in (1-19), (2-28) implies (1-17) and (2-29) implies (1-18).

We emphasize that Theorem 2.5 is valid up to times $\alpha^{2}$. (In fact, since the proof only relies on Proposition 2.2, it is valid up to times $\tau \alpha^{2}$ for an arbitrary $\tau>0$ with $C$ depending on $\tau$.) Consequently, the bounds in Theorem 1.3 are also valid up to times $\alpha^{2}$. However, since the evolved state and the main 
term in the approximation both have norm one, the bounds are only meaningful for times up to $\varepsilon \alpha$ for some small $\varepsilon>0$.

The basic intuition behind the bounds on $D_{k}, k=0, \ldots, 5$, is that each annihilation or creation operator is of order $\alpha^{-1}$ and therefore $D_{0}$, which contains only one creation operator, is of order $\alpha^{-1}$, $D_{1}, D_{2}, D_{3}, D_{4}$, which contain two creation or annihilation operators, are of order $\alpha^{-2}$ and $D_{5}$, which contains three creation or annihilation operators, is of order $\alpha^{-3}$. We illustrate this intuition in more detail in Section 2E with the simplest possible terms.

While this basic principle is true, it is oversimplifying the situation considerably as it does not take the slow-decaying terms $|k|^{-1}$ into account. The operator $\int e^{i k \cdot x} b_{k}^{*}|k|^{-1} d k$ and its adjoint are not bounded relative to the number operator $\int b_{k}^{*} b_{k} d x$. In fact, the treatment of these operators is the major difficulty that we have to overcome here.

At this point we have reduced the proof of Theorem 1.3 to the proof of Theorem 2.5, and the remainder of the paper is concerned with this. We bound $D_{0}$ in Section 3, $D_{1}$ in Section 4 and $D_{2}$ in Section 5. The terms $D_{3}, D_{4}$ and $D_{5}$, which are easier to bound than $D_{1}$ and $D_{2}$, are briefly discussed in Section 6 . Finally, the bounds (2-28) and (2-29) will be proved in Subsections 7A and 7B, respectively.

2D. A further decomposition. Using the fact that $P \underset{\tilde{\psi}_{t}}{\perp}=1-\left|\tilde{\psi}_{t}\right\rangle\left\langle\tilde{\psi}_{t}\right|$ (see the proof of Lemma 2.4), we have the decomposition

$$
F_{t, s}=F_{t, s}^{(1)}-F_{t, s}^{(2)}
$$

where

$$
F_{t, s}^{(1)}:=\int_{\mathbb{R}^{3}}\left(e^{i k \cdot x} W^{*}\left(\alpha^{2} \varphi_{t}\right) W\left(\alpha^{2} \varphi_{s}\right) b_{k}^{*} \tilde{\psi}_{s} \otimes \Omega\right) \frac{d k}{|k|}
$$

and, with the notation $\sigma_{\psi}$ from (2-2),

$$
F_{t, s}^{(2)}:=\tilde{\psi}_{s} \otimes W^{*}\left(\alpha^{2} \varphi_{t}\right) W\left(\alpha^{2} \varphi_{s}\right) b^{*}\left(\sigma_{\tilde{\psi}_{s}}\right) \Omega
$$

Correspondingly, we define

$$
D_{i}=D_{i 1}-D_{i 2} \quad \text { for } i=0,1,2,3,4,5 \text {. }
$$

In general, the terms $D_{i 2}$ are easier to deal with than the terms $D_{i 1}$. The reason for this is that $e^{i k \cdot x}|k|^{-1} \notin$ $L^{2}\left(\mathbb{R}^{3}\right)$, whereas $\sigma_{\tilde{\psi}_{t}} \in L^{2}\left(\mathbb{R}^{3}\right)$ by Lemma 2.1 , so the operator $\int e^{i k \cdot x} b_{k}^{*}|k|^{-1} d k$ in $F_{t, s}^{(1)}$ is harder to control than the operator $b^{*}\left(\sigma_{\tilde{\psi}_{s}}\right)$ in $F_{t, s}^{(2)}$.

For $k=1, \ldots, 5$, both operators $D_{i 1}$ and $D_{i 2}$ involve an operator $b_{k}^{*}, b_{k}$ or $b_{k}^{*} b_{k}$ to the left of $F_{t, s}^{(1)}$ or $F_{t, s}^{(2)}$, which in turn involves an operator $W^{*}\left(\alpha^{2} \varphi_{t}\right) W\left(\alpha^{2} \varphi_{s}\right)$. We now have the decomposition

$$
D_{i j}=D_{i j 1}+D_{i j 2} \quad \text { for } i=1,2,3,4,5 \text { and } j=1,2 \text {, }
$$

where $D_{i j 1}$ denotes the expression with $b_{k}, b_{k}^{*}$ or $b_{k}^{*} b_{k}$ commuted through the operator $W^{*}\left(\alpha^{2} \varphi_{t}\right) W\left(\alpha^{2} \varphi_{s}\right)$ and $D_{i j 2}$ denotes the expression coming from the commutator. To be explicit, we display some exemplary 
cases,

$$
\begin{aligned}
D_{111}= & \int_{0}^{t} \int_{0}^{t-s} \int_{\mathbb{R}^{3}} \int_{\mathbb{R}^{3}} e^{i \tilde{H}_{\varphi_{t}}\left(s+s_{1}\right)} e^{i k \cdot x} e^{-i H_{\varphi_{t}} s_{1}} e^{i k^{\prime} \cdot x} \\
& \times W^{*}\left(\alpha^{2} \varphi_{t}\right) W\left(\alpha^{2} \varphi_{s}\right) b_{k}^{*} b_{k^{\prime}}^{*} \tilde{\psi}_{s} \otimes \Omega \frac{d k^{\prime}}{\left|k^{\prime}\right|} \frac{d k}{|k|} d s_{1} d s, \\
D_{121}= & \int_{0}^{t} \int_{0}^{t-s} \int_{\mathbb{R}^{3}} e^{i \tilde{H}_{\varphi_{t}}\left(s+s_{1}\right)} e^{i k \cdot x} e^{-i H_{\varphi_{t}} s_{1}} W^{*}\left(\alpha^{2} \varphi_{t}\right) W\left(\alpha^{2} \varphi_{s}\right) b_{k}^{*} b^{*}\left(\sigma_{\tilde{\psi}_{s}}\right) \tilde{\psi}_{s} \otimes \Omega \frac{d k}{|k|} d s_{1} d s, \\
D_{211}= & \int_{0}^{t} \int_{0}^{t-s} \int_{\mathbb{R}^{3}} \int_{\mathbb{R}^{3}} e^{i \tilde{H}_{\varphi_{t}}\left(s+s_{1}\right)} e^{i k \cdot x} e^{-i H_{\varphi_{t}} s_{1}} e^{i k^{\prime} \cdot x} \\
D_{221}= & \int_{0}^{t} \int_{0}^{t-s} \int_{\mathbb{R}^{3}} e^{i \widetilde{H}_{\varphi_{t}}\left(s+s_{1}\right)} e^{i k \cdot x} e^{-i H_{\varphi_{t}} s_{1}} W^{*}\left(\alpha^{2} \varphi_{t}\right) W\left(\alpha^{2} \varphi_{s}\right) b_{k} b_{k^{\prime}}^{*} \tilde{\psi}_{s} \otimes \Omega \frac{d k^{\prime}}{\left|k^{\prime}\right|} \frac{d k}{|k|} d s_{1} d s,
\end{aligned}
$$

The commutator terms can be computed with the help of Corollary A.2. Recalling the definition of the function $g_{s, t}$ in (2-5), we have, for instance,

$$
\begin{aligned}
& D_{112}=-\int_{0}^{t} \int_{0}^{t-s} \int_{\mathbb{R}^{3}} e^{i \tilde{H}_{\varphi_{t}}\left(s+s_{1}\right)} g_{s, t} W^{*}\left(\alpha^{2} \varphi_{t}\right) W\left(\alpha^{2} \varphi_{s}\right) e^{-i H_{\varphi_{t}} s_{1}} e^{i k \cdot x} b_{k}^{*} \tilde{\psi}_{s} \otimes \Omega \frac{d k}{|k|} d s_{1} d s, \\
& D_{122}=-\int_{0}^{t} \int_{0}^{t-s} e^{i \tilde{H}_{\varphi_{t}}\left(s+s_{1}\right)} g_{s, t} W^{*}\left(\alpha^{2} \varphi_{t}\right) W\left(\alpha^{2} \varphi_{s}\right) e^{-i H_{\varphi_{t}} s_{1}} b^{*}\left(\sigma_{\tilde{\psi}_{s}}\right) \tilde{\psi}_{s} \otimes \Omega d s_{1} d s, \\
& D_{212}=-\int_{0}^{t} \int_{0}^{t-s} \int_{\mathbb{R}^{3}} e^{i \tilde{H}_{\varphi_{t}}\left(s+s_{1}\right)} \overline{g_{s, t}} W^{*}\left(\alpha^{2} \varphi_{t}\right) W\left(\alpha^{2} \varphi_{s}\right) e^{-i H_{\varphi_{t}} s_{1}} e^{i k \cdot x} b_{k}^{*} \tilde{\psi}_{s} \otimes \Omega \frac{d k}{|k|} d s_{1} d s, \\
& D_{222}=-\int_{0}^{t} \int_{0}^{t-s} e^{i \tilde{H}_{\varphi_{t}}\left(s+s_{1}\right)} \overline{g_{s, t}} W^{*}\left(\alpha^{2} \varphi_{t}\right) W\left(\alpha^{2} \varphi_{s}\right) e^{-i H_{\varphi_{t}} s_{1}} b^{*}\left(\sigma_{\tilde{\psi}_{s}}\right) \tilde{\psi}_{s} \otimes \Omega d s_{1} d s .
\end{aligned}
$$

2E. Some warm-up bounds. In order to prepare for the rather technical sections that follow, we will first focus on the terms that do not include a term of the form $|k|^{-1}$, that is, on the terms $D_{02}, D_{32}, D_{42}$ and $D_{52}$. We hope that this explains the underlying mechanism of our proof and the intuition that each annihilation or creation operator is of size $\alpha^{-1}$.

Bound on $\boldsymbol{D}_{\mathbf{0 2}}$. We recall that

$$
D_{02}=\int_{0}^{t}\left(e^{i H_{\varphi_{t}} s} \tilde{\psi}_{s}\right) \otimes\left(W^{*}\left(\alpha^{2} \varphi_{t}\right) W\left(\alpha^{2} \varphi_{s}\right) b^{*}\left(\sigma_{\tilde{\psi}_{s}}\right) \Omega\right) d s
$$

and, therefore, by Lemma 2.1 ,

$$
\left\|D_{02}\right\|_{\mathcal{L}^{2} \otimes \mathcal{F}} \leq \int_{0}^{t}\left\|\tilde{\psi}_{s}\right\|_{2}\left\|b^{*}\left(\sigma_{\widetilde{\psi}_{s}}\right) \Omega\right\|_{\mathcal{F}} d s=\alpha^{-1} \int_{0}^{t}\left\|\sigma_{\tilde{\psi}_{s}}\right\|_{2} d s \lesssim \alpha^{-1} t .
$$

Bound on $\boldsymbol{D}_{32}$. We have

$$
D_{321}=\int_{0}^{t} \int_{0}^{t-s} e^{i \widetilde{H}_{\varphi_{t}}\left(s+s_{1}\right)}\left(e^{-i H_{\varphi_{t}} s_{1}} \tilde{\psi}_{s}\right) \otimes\left(W^{*}\left(\alpha^{2} \varphi_{t}\right) W\left(\alpha^{2} \varphi_{s}\right) b^{*}\left(\varphi_{t}\right) b^{*}\left(\sigma_{\tilde{\psi}_{s}}\right) \Omega\right) d s_{1} d s
$$


and, according to Corollary A.2,

$$
D_{322}=-\int_{0}^{t} \int_{0}^{t-s} e^{i \tilde{H}_{\varphi_{t}}\left(s+s_{1}\right)}\left(e^{-i H_{\varphi_{t}} s_{1}} \tilde{\psi}_{s}\right) \otimes\left(\left(\varphi_{t}-\varphi_{s}, \varphi_{t}\right) W^{*}\left(\alpha^{2} \varphi_{t}\right) W\left(\alpha^{2} \varphi_{s}\right) b^{*}\left(\sigma_{\tilde{\psi}_{s}}\right) \Omega\right) d s_{1} d s .
$$

By the bounds from Lemma 2.1 we have

$$
\left\|b^{*}\left(\varphi_{t}\right) b^{*}\left(\sigma_{\tilde{\psi}_{s}}\right) \Omega\right\|_{\mathcal{F}}=\alpha^{-2}\left(\left\|\varphi_{t}\right\|_{2}^{2}\left\|\sigma_{\widetilde{\psi}_{s}}\right\|_{2}^{2}+\left|\left(\varphi_{t}, \sigma_{\widetilde{\psi}_{s}}\right)\right|^{2}\right)^{\frac{1}{2}} \lesssim \alpha^{-2}
$$

and therefore, using also the conservation of the $\mathcal{L}^{2}$-norm of $\tilde{\psi}_{s}$,

$$
\left\|D_{321}\right\|_{\mathcal{L}^{2} \otimes \mathcal{F}} \lesssim \alpha^{-2} t^{2}
$$

On the other hand, the bounds from Lemma 2.1 imply

$$
\left\|b^{*}\left(\sigma_{\tilde{\psi}_{s}}\right) \Omega\right\|_{\mathcal{F}}=\alpha^{-1}\left\|\sigma_{\tilde{\psi}_{s}}\right\|_{2} \lesssim \alpha^{-1}, \quad\left|\left(\varphi_{t}-\varphi_{s}, \varphi_{t}\right)\right| \lesssim \alpha^{-2}|t-s|,
$$

and therefore, using again the conservation of the $\mathcal{L}^{2}$-norm of $\tilde{\psi}_{s}$,

$$
\left\|D_{322}\right\|_{\mathcal{L}^{2} \otimes \mathcal{F}} \lesssim \alpha^{-3} t^{3} .
$$

Thus, we have shown that

$$
\left\|D_{32}\right\|_{\mathcal{L}^{2} \otimes \mathcal{F}} \lesssim \alpha^{-2} t^{2}\left(1+\alpha^{-1} t\right) .
$$

Bound on $D_{42}$. We have

$$
D_{421}=\int_{0}^{t} \int_{0}^{t-s} e^{i \tilde{H}_{\varphi_{t}}\left(s+s_{1}\right)}\left(e^{-i H_{\varphi_{t}} s_{1}} \tilde{\psi}_{s}\right) \otimes\left(W^{*}\left(\alpha^{2} \varphi_{t}\right) W\left(\alpha^{2} \varphi_{s}\right) b\left(\varphi_{t}\right) b^{*}\left(\sigma_{\tilde{\psi}_{s}}\right) \Omega\right) d s_{1} d s
$$

and, according to Corollary A.2,

$$
D_{422}=-\int_{0}^{t} \int_{0}^{t-s} e^{i \tilde{H}_{\varphi_{t}}\left(s+s_{1}\right)}\left(e^{-i H_{\varphi_{t}} s_{1}} \tilde{\psi}_{s}\right) \otimes\left(\left(\varphi_{t}, \varphi_{t}-\varphi_{s}\right) W^{*}\left(\alpha^{2} \varphi_{t}\right) W\left(\alpha^{2} \varphi_{s}\right) b^{*}\left(\sigma_{\tilde{\psi}_{s}}\right) \Omega\right) d s_{1} d s
$$

We commute once again and obtain

$$
D_{421}=\int_{0}^{t} \int_{0}^{t-s} e^{i \tilde{H}_{\varphi_{t}}\left(s+s_{1}\right)}\left(e^{-i H_{\varphi_{t}} s_{1}} \tilde{\psi}_{s}\right) \otimes\left(\alpha^{-2}\left(\varphi_{t}, \sigma_{\tilde{\psi}_{s}}\right) W^{*}\left(\alpha^{2} \varphi_{t}\right) W\left(\alpha^{2} \varphi_{s}\right) \Omega\right) d s_{1} d s .
$$

According to Lemma 2.1 we have $\left|\left(\varphi_{t}, \sigma_{\tilde{\psi}_{s}}\right)\right| \lesssim 1$. This and computations similar to those in the bound of $D_{32}$ yield

$$
\left\|D_{421}\right\|_{\mathcal{L}^{2} \otimes \mathcal{F}} \lesssim \alpha^{-2} t^{2}, \quad\left\|D_{422}\right\|_{\mathcal{L}^{2} \otimes \mathcal{F}} \lesssim \alpha^{-3} t^{3}
$$

Thus, we have shown that

$$
\left\|D_{42}\right\|_{\mathcal{L}^{2} \otimes \mathcal{F}} \lesssim \alpha^{-2} t^{2}\left(1+\alpha^{-1} t\right)
$$


Bound on $\boldsymbol{D}_{52}$. To simplify the notation, let us introduce

$$
\mathcal{N}:=\int_{\mathbb{R}^{3}} b_{k}^{*} b_{k} d k
$$

We have

$$
D_{521}=\int_{0}^{t} \int_{0}^{t-s} e^{i \tilde{H}_{\varphi_{t}}\left(s+s_{1}\right)}\left(e^{-i H_{\varphi_{t}} s_{1}} \tilde{\psi}_{s}\right) \otimes\left(W^{*}\left(\alpha^{2} \varphi_{t}\right) W\left(\alpha^{2} \varphi_{s}\right) \mathcal{N} b^{*}\left(\sigma_{\tilde{\psi}_{s}}\right) \Omega\right) d s_{1} d s .
$$

Moreover, by Corollary A.2,

$$
\begin{aligned}
{\left[\mathcal{N}, W^{*}\left(\alpha^{2} \varphi_{t}\right) W\left(\alpha^{2} \varphi_{s}\right)\right]=- } & W^{*}\left(\alpha^{2} \varphi_{t}\right) W\left(\alpha^{2} \varphi_{s}\right)\left(b\left(\varphi_{t}\right)-b\left(\varphi_{s}\right)\right) \\
& -W^{*}\left(\alpha^{2} \varphi_{t}\right) W\left(\alpha^{2} \varphi_{s}\right)\left(b^{*}\left(\varphi_{t}\right)-b^{*}\left(\varphi_{s}\right)\right)+W^{*}\left(\alpha^{2} \varphi_{t}\right) W\left(\alpha^{2} \varphi_{s}\right)\left\|\varphi_{t}-\varphi_{2}\right\|_{2}^{2}
\end{aligned}
$$

so

$$
\begin{aligned}
D_{522}= & \int_{0}^{t} \int_{0}^{t-s} e^{i \tilde{H}_{\varphi_{t}}\left(s+s_{1}\right)}\left(e^{-i H_{\varphi_{t}} s_{1}} \tilde{\psi}_{s}\right) \\
& \otimes\left(W^{*}\left(\alpha^{2} \varphi_{t}\right) W\left(\alpha^{2} \varphi_{s}\right)\left(-b\left(\varphi_{t}-\varphi_{s}\right)-b^{*}\left(\varphi_{t}-\varphi_{s}\right)+\left\|\varphi_{t}-\varphi_{2}\right\|_{2}^{2}\right) b^{*}\left(\sigma_{\tilde{\psi}_{s}}\right) \Omega\right) d s_{1} d s .
\end{aligned}
$$

We use $\mathcal{N} b^{*}\left(\sigma_{\tilde{\psi}_{s}}\right)=b^{*}\left(\sigma_{\tilde{\psi}_{s}}\right) \mathcal{N}+\alpha^{-2} b^{*}\left(\sigma_{\tilde{\psi}_{s}}\right)$ and obtain

$$
D_{521}=\alpha^{-2} \int_{0}^{t} \int_{0}^{t-s} e^{i \tilde{H}_{\varphi_{t}}\left(s+s_{1}\right)}\left(e^{-i H_{\varphi_{t}} s_{1}} \tilde{\psi}_{s}\right) \otimes\left(W^{*}\left(\alpha^{2} \varphi_{t}\right) W\left(\alpha^{2} \varphi_{s}\right) b^{*}\left(\sigma_{\tilde{\psi}_{s}}\right) \Omega\right) d s_{1} d s .
$$

Therefore, much as before,

$$
\left\|D_{521}\right\|_{\mathcal{L}^{2} \otimes \mathcal{F}} \lesssim \alpha^{-3} t^{2}
$$

For $D_{522}$ we commute again to get

$$
\begin{aligned}
D_{522}=\int_{0}^{t} \int_{0}^{t-s} & e^{i \tilde{H}_{\varphi_{t}}\left(s+s_{1}\right)}\left(e^{-i H_{\varphi_{t}} s_{1}} \tilde{\psi}_{s}\right) \otimes\left(W^{*}\left(\alpha^{2} \varphi_{t}\right) W\left(\alpha^{2} \varphi_{s}\right)\right. \\
& \left.\times\left(-\alpha^{-2}\left(\varphi_{t}-\varphi_{s}, \sigma_{\tilde{\psi}_{s}}\right) \Omega-b^{*}\left(\varphi_{t}-\varphi_{s}\right) b^{*}\left(\sigma_{\tilde{\psi}_{s}}\right) \Omega+\left\|\varphi_{t}-\varphi_{2}\right\|_{2}^{2} b^{*}\left(\sigma_{\tilde{\psi}_{s}}\right) \Omega\right)\right) d s_{1} d s .
\end{aligned}
$$

For the second term on the right side we compute

$$
\left\|b^{*}\left(\varphi_{t}-\varphi_{s}\right) b^{*}\left(\sigma_{\tilde{\psi}_{s}}\right) \Omega\right\|_{\mathcal{F}}=\alpha^{-2}\left(\left\|\varphi_{t}-\varphi_{s}\right\|_{2}^{2}\left\|\sigma_{\tilde{\psi}_{s}}\right\|_{2}^{2}+\left|\left(\varphi_{t}-\varphi_{s}, \sigma_{\tilde{\psi}_{s}}\right)\right|^{2}\right)^{\frac{1}{2}} .
$$

Using the bounds from Lemma 2.1 for $\left\|\varphi_{t}-\varphi_{s}\right\|_{2}$ we obtain that

$$
\left\|D_{522}\right\|_{\mathcal{L}^{2} \otimes \mathcal{F}} \lesssim \alpha^{-4} t^{3}\left(1+\alpha^{-1} t\right)
$$

Thus, we have shown that

$$
\left\|D_{52}\right\|_{\mathcal{L}^{2} \otimes \mathcal{F}} \lesssim \alpha^{-3} t^{2}\left(1+\alpha^{-2} t^{2}\right)
$$

\section{Bound on $D_{0}$}

We have already controlled $D_{02}$ in (2-38), so it remains to consider $D_{01}$. 
Bound on $\boldsymbol{D}_{\mathbf{0 1}}$. We recall that

$$
D_{01}=\int_{0}^{t} e^{i H_{\varphi_{t}} s} \int_{\mathbb{R}^{3}}\left(e^{i k \cdot x} W^{*}\left(\alpha^{2} \varphi_{t}\right) W\left(\alpha^{2} \varphi_{s}\right) b_{k}^{*} \tilde{\psi}_{s} \otimes \Omega\right) \frac{d k}{|k|} d s .
$$

The main difficulty here, which we will encounter in various forms throughout this paper, is the unboundedness of the operator $\int e^{i k \cdot x} b_{k}^{*}|k|^{-1} d k$ (for any fixed $x \in \mathbb{R}^{3}$ ), since $e^{i k \cdot x}|k|^{-1} \notin \mathcal{L}^{2}\left(\mathbb{R}^{3}\right.$ ).

To overcome this difficulty we make use of the oscillatory behavior of $e^{i k \cdot x}$ via the formula

$$
e^{i k \cdot x}=\frac{1-i k \cdot \nabla_{x}}{1+|k|^{2}} e^{i k \cdot x}
$$

and aim at integrating by parts with respect to $x$. However, this integration by parts creates a new difficulty: the resulting operator $\nabla_{x}$ is unbounded and has to be controlled.

To overcome this new difficulty, it will be desirable to have an operator $(-\Delta+1)^{-1}$ somewhere in the expression of $D_{01}$ so that we can use it to control $\nabla_{x}$, since obviously $\nabla_{x}(-\Delta+1)^{-1}$ is bounded. It is equivalent and technically more convenient to work with $\left(H_{\varphi_{t}}+M\right)^{-1}$, where $M>0$ is a large constant (independent of $\alpha$ and $t$ ), instead of $(-\Delta+1)^{-1}$. In order to create this term we first integrate by parts in $s$ and make use of the identity

$$
e^{i H_{\varphi_{t}} s}=-i\left(H_{\varphi_{t}}+M\right)^{-1} e^{-i M s} \partial_{s}\left[e^{i\left(H_{\varphi_{t}}+M\right) s}\right] .
$$

We obtain, using the fact that $H_{\varphi_{t}}$ commutes with $W\left(\alpha^{2} \varphi_{s}\right)$,

$$
\begin{aligned}
& D_{01}=- i e^{i H_{\varphi_{t}} t}\left(H_{\varphi_{t}}+M\right)^{-1} \int_{\mathbb{R}^{3}} e^{i k \cdot x} b_{k}^{*} \tilde{\psi}_{t} \otimes \Omega \frac{d k}{|k|} \\
&+ i W^{*}\left(\alpha^{2} \varphi_{t}\right) W\left(\alpha^{2} \varphi_{0}\right)\left(H_{\varphi_{t}}+M\right)^{-1} \int_{\mathbb{R}^{3}} e^{i k \cdot x} b_{k}^{*} \tilde{\psi}_{0} \otimes \Omega \frac{d k}{|k|} \\
&+ M \int_{0}^{t} e^{i H_{\varphi_{t}} s} W^{*}\left(\alpha^{2} \varphi_{t}\right) W\left(\alpha^{2} \varphi_{s}\right)\left(H_{\varphi_{t}}+M\right)^{-1} \int_{\mathbb{R}^{3}} e^{i k \cdot x} b_{k}^{*} \tilde{\psi}_{s} \otimes \Omega \frac{d k}{|k|} d s \\
&+ i \int_{0}^{t} e^{i H_{\varphi_{t}} s} W^{*}\left(\alpha^{2} \varphi_{t}\right) W\left(\alpha^{2} \varphi_{s}\right)\left(H_{\varphi_{t}}+M\right)^{-1} \int_{\mathbb{R}^{3}} e^{i k \cdot x} b_{k}^{*} \partial_{s} \tilde{\psi}_{s} \otimes \Omega \frac{d k}{|k|} d s \\
&+i \int_{0}^{t} e^{i H_{\varphi_{t}} s} W^{*}\left(\alpha^{2} \varphi_{t}\right)\left(\partial_{s} W\left(\alpha^{2} \varphi_{s}\right)\right)\left(H_{\varphi_{t}}+M\right)^{-1} \int_{\mathbb{R}^{3}} e^{i k \cdot x} b_{k}^{*} \tilde{\psi}_{s} \otimes \Omega \frac{d k}{|k|} d s \\
&=D_{011}+D_{012}+D_{013}+D_{014}+D_{015},
\end{aligned}
$$

where the terms $D_{01 k}$ are defined in a natural way. We will prove the following lemma.

Lemma 3.1. For $u \in \mathcal{H}^{1}\left(\mathbb{R}^{3}\right)$ and $f \in \mathcal{L}^{2}\left(\mathbb{R}^{3}\right)$,

$$
\left\|(-\Delta+1)^{-\frac{1}{2}} \int_{\mathbb{R}^{3}} e^{i k \cdot x} b_{k}^{*} u \otimes \Omega \frac{d k}{|k|}\right\|_{\mathcal{L}^{2} \otimes \mathcal{F}} \lesssim \alpha^{-1}\|u\|_{\mathcal{H}^{1}}
$$

and

$$
\left\|(-\Delta+1)^{-\frac{1}{2}} \int_{\mathbb{R}^{3}} e^{i k \cdot x} b^{*}(f) b_{k}^{*} u \otimes \Omega \frac{d k}{|k|}\right\|_{\mathcal{L}^{2} \otimes \mathcal{F}} \lesssim \alpha^{-2}\|u\|_{\mathcal{H}^{1}}\|f\|_{2} .
$$


We defer the proof of this lemma to the end of this section and first show how to use it to control $D_{01}$. By Corollary B.2 and Lemma 2.1, we can choose $M$ large enough so that $\left(H_{\varphi_{t}}+M\right)^{-\frac{1}{2}}(-\Delta+1)^{\frac{1}{2}}$ is bounded uniformly in $t \in \mathbb{R}$. Moreover, by Proposition 2.2, $\tilde{\psi}_{t}$ and $\partial_{t} \tilde{\psi}_{t}$ belong to $H^{1}\left(\mathbb{R}^{3}\right)$ and have uniformly bounded norms for $t \in\left[0, \alpha^{2}\right]$; see also the remark at the beginning of Section $2 \mathrm{~B}$ concerning the bounds on $\partial_{t} \tilde{\psi}_{t}$. These facts, together with the unitarity of $e^{i H_{\varphi_{t}} s}, W^{*}\left(\alpha^{2} \varphi_{t}\right)$ and $W\left(\alpha^{2} \varphi_{s}\right)$, imply that

$$
\left\|D_{011}\right\|_{\mathcal{L}^{2} \otimes \mathcal{F}} \lesssim \alpha^{-1}, \quad\left\|D_{012}\right\|_{\mathcal{L}^{2} \otimes \mathcal{F}} \lesssim \alpha^{-1}
$$

and

$$
\left\|D_{013}\right\|_{\mathcal{L}^{2} \otimes \mathcal{F}} \lesssim \alpha^{-1} t, \quad\left\|D_{014}\right\|_{\mathcal{L}^{2} \otimes \mathcal{F}} \lesssim \alpha^{-1} t .
$$

In order to deal with the term $D_{015}$ we make use of (A-4) and find

$$
\begin{aligned}
D_{015}=- & \int_{0}^{t}\left(\operatorname{Im}\left(\varphi_{s}, \alpha^{2} \partial_{s} \varphi_{s}\right)\right) e^{i H_{\varphi_{t}} s} W^{*}\left(\alpha^{2} \varphi_{t}\right) W\left(\alpha^{2} \varphi_{s}\right)\left(H_{\varphi_{t}}+M\right)^{-1} \int_{\mathbb{R}^{3}} e^{i k \cdot x} b_{k}^{*} \tilde{\psi}_{s} \otimes \Omega \frac{d k}{|k|} d s \\
& +i \int_{0}^{t} e^{i H_{\varphi_{t}} s} W^{*}\left(\alpha^{2} \varphi_{t}\right) W\left(\alpha^{2} \varphi_{s}\right)\left(H_{\varphi_{t}}+M\right)^{-1} \int_{\mathbb{R}^{3}} e^{i k \cdot x} b^{*}\left(\alpha^{2} \partial_{s} \varphi_{s}\right) b_{k}^{*} \tilde{\psi}_{s} \otimes \Omega \frac{d k}{|k|} d s \\
& \quad-i \int_{0}^{t} e^{i H_{\varphi_{t}} s} W^{*}\left(\alpha^{2} \varphi_{t}\right) W\left(\alpha^{2} \varphi_{s}\right)\left(H_{\varphi_{t}}+M\right)^{-1} \int_{\mathbb{R}^{3}} e^{i k \cdot x} b\left(\alpha^{2} \partial_{s} \varphi_{s}\right) b_{k}^{*} \tilde{\psi}_{s} \otimes \Omega \frac{d k}{|k|} d s \\
= & D_{0151}+D_{0152}+D_{0153} .
\end{aligned}
$$

From Lemma 2.1 we know that $\left|\left(\varphi_{s}, \alpha^{2} \partial_{s} \varphi_{s}\right)\right| \lesssim 1$ and $\left\|\alpha^{2} \partial_{s} \varphi_{s}\right\| \lesssim 1$. Thus, the first and the second bounds in Lemma 3.1 imply, respectively,

$$
\left\|D_{0151}\right\|_{\mathcal{L}^{2} \otimes \mathcal{F}} \lesssim \alpha^{-1} t, \quad\left\|D_{0152}\right\|_{\mathcal{L}^{2} \otimes \mathcal{F}} \lesssim \alpha^{-2} t .
$$

For $D_{0153}$ we use the commutation relations to rewrite it as

$$
D_{0153}=-i \int_{0}^{t} e^{i H_{\varphi_{t}} s} W^{*}\left(\alpha^{2} \varphi_{t}\right) W\left(\alpha^{2} \varphi_{s}\right)\left(H_{\varphi_{t}}+M\right)^{-1} g_{s} \tilde{\psi}_{s} \otimes \Omega d s
$$

with $g_{s}$ from (2-6). Therefore, Proposition 2.2 yields

$$
\left\|D_{0153}\right\|_{\mathcal{L}^{2} \otimes \mathcal{F}} \lesssim \alpha^{-2} t
$$

To summarize, we have shown that

$$
\left\|D_{01}\right\|_{\mathcal{L}^{2} \otimes \mathcal{F}} \lesssim \alpha^{-1}(1+t) .
$$

Proof of Lemma 3.1. For any $\gamma \in \mathcal{L}^{2}\left(\mathbb{R}^{3}\right) \otimes \mathcal{F}$ and $\left(\Phi_{k}\right)_{k \in \mathbb{R}^{3}} \subset \mathcal{F}$, we use (3-1) to find

$$
\begin{aligned}
\left\langle\gamma,(-\Delta+1)^{-\frac{1}{2}} \int_{\mathbb{R}^{3}} e^{i k \cdot x} u \otimes \Phi_{k} \frac{d k}{|k|}\right\rangle_{\mathcal{L}^{2} \otimes \mathcal{F}}=\left\langle\nabla(-\Delta+1)^{-\frac{1}{2}} \gamma, \int_{\mathbb{R}^{3}} \frac{i k e^{i k \cdot x}}{|k|\left(1+|k|^{2}\right)} u \otimes \Phi_{k} d k\right\rangle_{\mathcal{L}^{2} \otimes \mathcal{F}} \\
+\left\langle(-\Delta+1)^{-\frac{1}{2}} \gamma, \int_{\mathbb{R}^{3}} \frac{i k e^{i k \cdot x}}{|k|\left(1+|k|^{2}\right)}(\nabla u) \otimes \Phi_{k} d k\right\rangle_{\mathcal{L}^{2} \otimes \mathcal{F}} \\
+\left\langle(-\Delta+1)^{-\frac{1}{2}} \gamma, \int_{\mathbb{R}^{3}} \frac{e^{i k \cdot x}}{|k|\left(1+|k|^{2}\right)} u \otimes \Phi_{k} d k\right\rangle_{\mathcal{L}^{2} \otimes \mathcal{F}} .
\end{aligned}
$$


Clearly,

$$
\left\|\nabla(-\Delta+1)^{-\frac{1}{2}} \gamma\right\|_{\mathcal{L}^{2} \otimes \mathcal{F}} \leq\|\gamma\|_{\mathcal{L}^{2} \otimes \mathcal{F}} \quad \text { and } \quad\left\|(-\Delta+1)^{-\frac{1}{2}} \gamma\right\|_{\mathcal{L}^{2} \otimes \mathcal{F}} \leq\|\gamma\|_{\mathcal{L}^{2} \otimes \mathcal{F}}
$$

so

$$
\begin{aligned}
\|(-\Delta+1)^{-\frac{1}{2}} \int_{\mathbb{R}^{3}} e^{i k \cdot x} u & \otimes \Phi_{k} \frac{d k}{|k|} \|_{\mathcal{L}^{2} \otimes \mathcal{F}} \\
& \lesssim\|u\|_{\mathcal{H}^{1}} \sup _{x \in \mathbb{R}^{d}}\left(\left\|\int_{\mathbb{R}^{3}} \frac{i k e^{i k \cdot x}}{|k|\left(1+|k|^{2}\right)} \Phi_{k} d k\right\|_{\mathcal{F}}+\left\|\int_{\mathbb{R}^{3}} \frac{e^{i k \cdot x}}{|k|\left(1+|k|^{2}\right)} \Phi_{k} d k\right\|_{\mathcal{F}}\right) .
\end{aligned}
$$

If $\Phi_{k}=b_{k}^{*} \Omega$, we use the fact that

$$
\frac{1}{|k|\left(1+|k|^{2}\right)}, \frac{k}{|k|\left(1+|k|^{2}\right)} \in \mathcal{L}^{2}\left(\mathbb{R}^{3}\right)
$$

to conclude that, uniformly in $x \in \mathbb{R}^{3}$,

$$
\left\|\int_{\mathbb{R}^{3}} \frac{i k e^{i k \cdot x}}{|k|\left(1+|k|^{2}\right)} b_{k}^{*} \Omega d k\right\|_{\mathcal{F}} \lesssim \alpha^{-1}, \quad\left\|\int_{\mathbb{R}^{3}} \frac{e^{i k \cdot x}}{|k|\left(1+|k|^{2}\right)} b_{k}^{*} \Omega d k\right\|_{\mathcal{F}} \lesssim \alpha^{-1} .
$$

This proves the first bound in the lemma. If $\Phi_{k}=b^{*}(f) b_{k}^{*} \Omega$, one can similarly show that

$$
\left\|\int_{\mathbb{R}^{3}} \frac{i k e^{i k \cdot x}}{|k|\left(1+|k|^{2}\right)} b^{*}(f) b_{k}^{*} \Omega d k\right\|_{\mathcal{F}} \lesssim \frac{\|f\|_{2}}{\alpha^{2}}, \quad\left\|\int_{\mathbb{R}^{3}} \frac{e^{i k \cdot x}}{|k|\left(1+|k|^{2}\right)} b^{*}(f) b_{k}^{*} \Omega d k\right\|_{\mathcal{F}} \lesssim \frac{\|f\|_{2}}{\alpha^{2}} .
$$

This proves the second bound in the lemma.

\section{Bound on $D_{1}$}

Bound on $D_{111}$. We recall equation (2-30) for $D_{111}$. In this equation, we commute $e^{i k \cdot x}$ with $e^{-i H_{\varphi t} s}$. Thus, if we introduce the operator

$$
H_{\varphi}(k):=e^{i k \cdot x} H_{\varphi} e^{-i k \cdot x}=\left(i \nabla_{x}+k\right)^{2}+V_{\varphi}+\int_{\mathbb{R}^{3}}|\varphi(k)|^{2} d k,
$$

we obtain

$$
\begin{aligned}
D_{111}=\int_{0}^{t} \int_{0}^{t-s} \int_{\mathbb{R}^{3}} \int_{\mathbb{R}^{3}} e^{i \widetilde{H}_{\varphi_{t}}\left(s+s_{1}\right)} e^{-i H_{\varphi_{t}}(k) s_{1}} e^{i\left(k+k^{\prime}\right) \cdot x} & \\
& \times W^{*}\left(\alpha^{2} \varphi_{t}\right) W\left(\alpha^{2} \varphi_{s}\right) b_{k}^{*} b_{k^{\prime}}^{*} \tilde{\psi}_{s} \otimes \Omega \frac{d k^{\prime}}{\left|k^{\prime}\right|} \frac{d k}{|k|} d s_{1} d s .
\end{aligned}
$$

Controlling $D_{111}$ is harder than controlling $D_{01}$ because there are two slowly decaying terms $|k|^{-1}$ and $\left|k^{\prime}\right|^{-1}$. The beginning of the proof, however, is similar; namely, for a large constant $M>0$ to be specified, independent of $t$ and $\alpha$, we integrate by parts in $s$ using

$$
e^{i \widetilde{H}_{\varphi_{t}} s}=-i\left(\widetilde{H}_{\varphi_{t}}+M\right)^{-1} e^{-i M s}\left[\partial_{s} e^{i\left(\widetilde{H}_{\varphi_{t}}+M\right) s}\right] .
$$


In this way we obtain

$$
\begin{aligned}
& D_{111}=-i \int_{0}^{t} \int_{\mathbb{R}^{3}} \int_{\mathbb{R}^{3}} e^{i \tilde{H}_{\varphi_{t}} t}\left(\tilde{H}_{\varphi_{t}}+M\right)^{-1} e^{-i H_{\varphi_{t}}(k) s_{1}} e^{i\left(k^{\prime}+k\right) \cdot x} \\
& +i \int_{0}^{t} \int_{\mathbb{R}^{3}} \int_{\mathbb{R}^{3}} e^{i \tilde{H}_{\varphi_{t}} s_{1}}\left(\tilde{H}_{\varphi_{t}}+M\right)^{-1} e^{-i H_{\varphi_{t}}(k) s_{1}} e^{i\left(k^{\prime}+k\right) \cdot x} \\
& \times W^{*}\left(\alpha^{2} \varphi_{t}\right) W\left(\alpha^{2} \varphi_{t-s_{1}}\right) b_{k}^{*} b_{k^{\prime}}^{*} \tilde{\psi}_{t-s_{1}} \otimes \Omega \frac{d k^{\prime}}{\left|k^{\prime}\right|} \frac{d k}{|k|} d s_{1} \\
& \times W^{*}\left(\alpha^{2} \varphi_{t}\right) W\left(\alpha^{2} \varphi_{0}\right) b_{k}^{*} b_{k^{\prime}}^{*} \tilde{\psi}_{0} \otimes \Omega \frac{d k^{\prime}}{\left|k^{\prime}\right|} \frac{d k}{|k|} d s_{1} \\
& +M \int_{0}^{t} \int_{0}^{t-s} \int_{\mathbb{R}^{3}} \int_{\mathbb{R}^{3}} e^{i \tilde{H}_{\varphi_{t}}\left(s+s_{1}\right)}\left(\tilde{H}_{\varphi_{t}}+M\right)^{-1} e^{-i H_{\varphi_{t}}(k) s_{1}} e^{i\left(k^{\prime}+k\right) \cdot x} \\
& \times W^{*}\left(\alpha^{2} \varphi_{t}\right) W\left(\alpha^{2} \varphi_{s}\right) b_{k}^{*} b_{k^{\prime}}^{*} \tilde{\psi}_{s} \otimes \Omega \frac{d k^{\prime}}{\left|k^{\prime}\right|} \frac{d k}{|k|} d s_{1} d s \\
& +i \int_{0}^{t} \int_{0}^{t-s} \int_{\mathbb{R}^{3}} \int_{\mathbb{R}^{3}} e^{i \tilde{H}_{\varphi_{t}}\left(s+s_{1}\right)}\left(\tilde{H}_{\varphi_{t}}+M\right)^{-1} e^{-i H_{\varphi_{t}}(k) s_{1}} e^{i\left(k^{\prime}+k\right) \cdot x} \\
& \times W^{*}\left(\alpha^{2} \varphi_{t}\right) W\left(\alpha^{2} \varphi_{s}\right) b_{k}^{*} b_{k^{\prime}}^{*}\left[\partial_{s} \tilde{\psi}_{s}\right] \otimes \Omega \frac{d k^{\prime}}{\left|k^{\prime}\right|} \frac{d k}{|k|} d s_{1} d s \\
& +i \int_{0}^{t} \int_{0}^{t-s} \int_{\mathbb{R}^{3}} \int_{\mathbb{R}^{3}} e^{i \tilde{H}_{\varphi_{t}}\left(s+s_{1}\right)}\left(\tilde{H}_{\varphi_{t}}+M\right)^{-1} e^{-i H_{\varphi_{t}}(k) s_{1}} e^{i\left(k^{\prime}+k\right) \cdot x} \\
& \times W^{*}\left(\alpha^{2} \varphi_{t}\right)\left[\partial_{s} W\left(\alpha^{2} \varphi_{s}\right)\right] b_{k}^{*} b_{k^{\prime}}^{*} \tilde{\psi}_{s} \otimes \Omega \frac{d k^{\prime}}{\left|k^{\prime}\right|} \frac{d k}{|k|} d s_{1} d s .
\end{aligned}
$$

We now use (2-13), which implies

$$
\begin{aligned}
\left(\tilde{H}_{\varphi_{t}}+M\right)^{-1} W^{*}\left(\alpha^{2} \varphi_{t}\right) W\left(\alpha^{2} \varphi_{s}\right) & =W^{*}\left(\alpha^{2} \varphi_{t}\right)\left(\tilde{H}_{\alpha}^{F}+M\right)^{-1} W\left(\alpha^{2} \varphi_{s}\right) \\
& =W^{*}\left(\alpha^{2} \varphi_{t}\right) W\left(\alpha^{2} \varphi_{s}\right)\left(\tilde{H}_{\varphi_{s}}+M\right)^{-1},
\end{aligned}
$$

in order to commute $\left(\tilde{H}_{\varphi_{t}}+M\right)^{-1}$ to the right through $W^{*}\left(\alpha^{2} \varphi_{t}\right) W\left(\alpha^{2} \varphi_{s}\right)$. Moreover, we use Lemma A.3 to compute $\partial_{s} W\left(\alpha^{2} \varphi_{s}\right)$. In this way we obtain

with

$$
\begin{aligned}
& D_{111}=-i \int_{0}^{t} e^{i \tilde{H}_{\varphi_{t}} t} W^{*}\left(\alpha^{2} \varphi_{t}\right) W\left(\alpha^{2} \varphi_{s}\right) Q_{1} d s \\
& +i \int_{0}^{t} e^{i \widetilde{H}_{\varphi_{t}} s_{1}} W^{*}\left(\alpha^{2} \varphi_{t}\right) W\left(\alpha^{2} \varphi_{0}\right) Q_{2} d s_{1} \\
& \quad+M \int_{0}^{t} \int_{0}^{t-s} e^{i \widetilde{H}_{\varphi_{t}}\left(s+s_{1}\right)} W^{*}\left(\alpha^{2} \varphi_{t}\right) W\left(\alpha^{2} \varphi_{s}\right) Q_{3} d s_{1} d s \\
& \quad+i \int_{0}^{t} \int_{0}^{t-s} e^{i \tilde{H}_{\varphi_{t}}\left(s+s_{1}\right)} W^{*}\left(\alpha^{2} \varphi_{t}\right) W\left(\alpha^{2} \varphi_{s}\right) Q_{4} d s_{1} d s \\
& \quad+i \int_{0}^{t} \int_{0}^{t-s} e^{i \tilde{H}_{\varphi_{t}}\left(s+s_{1}\right)} W^{*}\left(\alpha^{2} \varphi_{t}\right) W\left(\alpha^{2} \varphi_{s}\right) Q_{5} d s_{1} d s
\end{aligned}
$$

$$
\begin{aligned}
Q_{1} & :=\left(\tilde{H}_{\varphi_{s}}+M\right)^{-1} \int_{\mathbb{R}^{3}} \int_{\mathbb{R}^{3}} e^{-i H_{\varphi_{t}}(k)(t-s)} e^{i\left(k^{\prime}+k\right) \cdot x} b_{k}^{*} b_{k^{\prime}}^{*} \tilde{\psi}_{s} \otimes \Omega \frac{d k^{\prime}}{\left|k^{\prime}\right|} \frac{d k}{|k|}, \\
Q_{2} & :=\left(\tilde{H}_{\varphi_{0}}+M\right)^{-1} \int_{\mathbb{R}^{3}} \int_{\mathbb{R}^{3}} e^{-i H_{\varphi_{t}}(k) s_{1}} e^{i\left(k^{\prime}+k\right) \cdot x} b_{k}^{*} b_{k^{\prime}}^{*} \tilde{\psi}_{0} \otimes \Omega \frac{d k^{\prime}}{\left|k^{\prime}\right|} \frac{d k}{|k|},
\end{aligned}
$$




$$
\begin{aligned}
& Q_{3}:=\left(\tilde{H}_{\varphi_{s}}+M\right)^{-1} \int_{\mathbb{R}^{3}} \int_{\mathbb{R}^{3}} e^{-i H_{\varphi_{t}}(k) s_{1}} e^{i\left(k^{\prime}+k\right) \cdot x} b_{k}^{*} b_{k^{\prime}}^{*} \tilde{\psi}_{s} \otimes \Omega \frac{d k^{\prime}}{\left|k^{\prime}\right|} \frac{d k}{|k|}, \\
& Q_{4}:=\left(\tilde{H}_{\varphi_{s}}+M\right)^{-1} \int_{\mathbb{R}^{3}} \int_{\mathbb{R}^{3}} e^{-i H_{\varphi_{t}}(k) s_{1}} e^{i\left(k^{\prime}+k\right) \cdot x} b_{k}^{*} b_{k^{\prime}}^{*}\left[\partial_{s} \tilde{\psi}_{s}\right] \otimes \Omega \frac{d k^{\prime}}{\left|k^{\prime}\right|} \frac{d k}{|k|}, \\
& Q_{5}:=\left(\tilde{H}_{\varphi_{s}}+M\right)^{-1} \int_{\mathbb{R}^{3}} \int_{\mathbb{R}^{3}} e^{-i H_{\varphi_{t}}(k) s_{1}} e^{i\left(k^{\prime}+k\right) \cdot x}\left(b^{*}\left(\alpha^{2} \partial_{s} \varphi_{s}\right)-b\left(\alpha^{2} \partial_{s} \varphi_{s}\right)\right. \\
&\left.\quad+i \operatorname{Im}\left(\varphi_{s}, \alpha^{2} \partial_{s} \varphi_{s}\right)\right) b_{k}^{*} b_{k^{\prime}}^{*} \tilde{\psi}_{s} \otimes \Omega \frac{d k^{\prime}}{\left|k^{\prime}\right|} \frac{d k}{|k|} .
\end{aligned}
$$

(Here, we suppress the dependence on $t, s$ and $s_{1}$ in the notation of the $Q_{j}$.)

In the remainder of this section we shall show that, uniformly for $0 \leq s, s_{1} \leq t \leq \alpha^{2}$,

$$
\left\|Q_{j}\right\|_{\mathcal{L}^{2} \otimes \mathcal{F}} \lesssim \alpha^{-2} \quad \text { if } j=1,2,3,4,5 .
$$

This will imply that

$$
\left\|D_{111}\right\|_{\mathcal{L}^{2} \otimes \mathcal{F}} \lesssim \alpha^{-2} t(1+t) .
$$

Since the operator $\left(\widetilde{H}_{\varphi_{s}}+M\right)^{-1}(-\Delta+\mathcal{N}+M)$ is not bounded, bounding the $Q_{j}$ is rather involved. (Here $\mathcal{N}$ was introduced in (2-41).) With the notation

$$
Z_{\varphi}:=V_{\varphi}+\int_{\mathbb{R}^{3}}|\varphi(x)|^{2} d k+\int_{\mathbb{R}^{3}}\left(e^{-i k \cdot x} b_{k}+e^{i k \cdot x} b_{k}^{*}\right) \frac{d k}{|k|}+b(\varphi)+b^{*}(\varphi),
$$

we abbreviate (2-14) as

$$
\tilde{H}_{\varphi}=-\Delta+\mathcal{N}+Z_{\varphi}
$$

Defining

$$
\tilde{Z}_{\varphi}:=(-\Delta+\mathcal{N}+M)^{-\frac{1}{2}} Z_{\varphi}(-\Delta+\mathcal{N}+M)^{-\frac{1}{2}},
$$

we have

$$
\begin{aligned}
\left(\widetilde{H}_{\varphi}+M\right)^{-1} & =(-\Delta+\mathcal{N}+M)^{-\frac{1}{2}}\left(1+\widetilde{Z}_{\varphi}\right)^{-1}(-\Delta+\mathcal{N}+M)^{-\frac{1}{2}} \\
& =(-\Delta+\mathcal{N}+M)^{-1}-(-\Delta+\mathcal{N}+M)^{-\frac{1}{2}}\left(1+\widetilde{Z}_{\varphi}\right)^{-1}(-\Delta+\mathcal{N}+M)^{-\frac{1}{2}} Z_{\varphi}(-\Delta+\mathcal{N}+M)^{-1} .
\end{aligned}
$$

It is not difficult to see that for every $\varepsilon>0$ and $A>0$ there is an $M$ such that

$$
\left\|\widetilde{Z}_{\varphi}\right\|_{\mathcal{L}^{2} \otimes \mathcal{F} \mapsto \mathcal{L}^{2} \otimes F} \leq \varepsilon
$$

for all $\varphi$ with $\|\varphi\|_{\mathcal{L}^{2}} \leq A$; for details of this argument we refer to [Frank and Schlein 2014]. Thus, using the bound on $\left\|\varphi_{s}\right\|_{\mathcal{L}^{2}}$ from Lemma 2.1, we can choose $M$ in such a way that

$$
\left\|\widetilde{Z}_{\varphi_{s}}\right\|_{\mathcal{L}^{2} \otimes \mathcal{F} \mapsto \mathcal{L}^{2} \otimes F} \leq \frac{1}{2} \quad \text { for all } s>0 .
$$

Therefore, the operator $1+\widetilde{Z}_{\varphi_{s}}$ in the above formula for $\left(H_{\varphi_{s}}+M\right)^{-1}$ is invertible. We use this formula to decompose

$$
\begin{gathered}
Q_{1}=\left(1-(-\Delta+\mathcal{N}+M)^{-\frac{1}{2}}\left(1+\widetilde{Z}_{\varphi_{s}}\right)^{-1}(-\Delta+\mathcal{N}+M)^{-\frac{1}{2}}\left(V_{\varphi_{s}}+\int_{\mathbb{R}^{3}}\left|\varphi_{S}(x)\right|^{2} d k+b\left(\varphi_{s}\right)+b^{*}\left(\varphi_{s}\right)\right)\right) Q_{10} \\
-(-\Delta+\mathcal{N}+M)^{-\frac{1}{2}}\left(1+\widetilde{Z}_{\varphi_{s}}\right)^{-1}\left(Q_{11}+Q_{12}\right)
\end{gathered}
$$


with

$$
\begin{aligned}
& Q_{10}:=(-\Delta+\mathcal{N}+M)^{-1} \int_{\mathbb{R}^{3}} \int_{\mathbb{R}^{3}} e^{-i H_{\varphi t}(k)(t-s)} e^{i\left(k^{\prime}+k\right) \cdot x} b_{k}^{*} b_{k^{\prime}}^{*} \tilde{\psi}_{s} \otimes \Omega \frac{d k^{\prime}}{\left|k^{\prime}\right|} \frac{d k}{|k|}, \\
& \begin{aligned}
& Q_{11}:=(-\Delta+\mathcal{N}+M)^{-\frac{1}{2}}\left(\int_{\mathbb{R}^{3}} e^{-i k^{\prime \prime} \cdot x} b_{k^{\prime \prime}}\right.\left.\frac{d k^{\prime \prime}}{\left|k^{\prime \prime}\right|}\right)(-\Delta+\mathcal{N}+M)^{-1} \\
& \times \int_{\mathbb{R}^{3}} \int_{\mathbb{R}^{3}} e^{-i H_{\varphi_{t}}(k)(t-s)} e^{i\left(k^{\prime}+k\right) \cdot x} b_{k}^{*} b_{k^{\prime}}^{*} \tilde{\psi}_{s} \otimes \Omega \frac{d k^{\prime}}{\left|k^{\prime}\right|} \frac{d k}{|k|}, \\
& Q_{12}:=(-\Delta+\mathcal{N}+M)^{-\frac{1}{2}}\left(\int_{\mathbb{R}^{3}} e^{i k^{\prime \prime} \cdot x} b_{k^{\prime \prime}}^{*} \frac{d k^{\prime \prime}}{\left|k^{\prime \prime}\right|}\right)(-\Delta+\mathcal{N}+M)^{-1} \\
& \\
& \times \int_{\mathbb{R}^{3}} \int_{\mathbb{R}^{3}} e^{-i H_{\varphi t}(k)(t-s)} e^{i\left(k^{\prime}+k\right) \cdot x} b_{k}^{*} b_{k^{\prime}}^{*} \tilde{\psi}_{s} \otimes \Omega \frac{d k^{\prime}}{\left|k^{\prime}\right|} \frac{d k}{|k|} .
\end{aligned}
\end{aligned}
$$

Using (4-4), the fact that $(-\Delta+\mathcal{N}+M)^{-\frac{1}{2}}\left(b\left(\varphi_{s}\right)+b^{*}\left(\varphi_{s}\right)\right)$ is bounded uniformly in $s$, as well as the estimates $\left\|V_{\varphi_{s}}\right\|_{\infty} \lesssim 1$ (from (C-1) and Proposition 2.2) and $\left\|\varphi_{s}\right\|_{2} \lesssim 1$ (from Lemma 2.1), we conclude from (4-5) that

$$
\left\|Q_{1}\right\|_{\mathcal{L}^{2} \otimes \mathcal{F}} \lesssim\left\|Q_{10}\right\|_{\mathcal{L}^{2} \otimes \mathcal{F}}+\left\|Q_{11}\right\|_{\mathcal{L}^{2} \otimes \mathcal{F}}+\left\|Q_{12}\right\|_{\mathcal{L}^{2} \otimes \mathcal{F}}
$$

We now bound the three terms on the right side separately.

Bound on $Q_{10}$. To control $Q_{10}$ we prove an analogue of Lemma 3.1 for the case of two singularities.

Lemma 4.1. For $u \in \mathcal{H}^{2}\left(\mathbb{R}^{3}\right), f \in \mathcal{L}^{2}\left(\mathbb{R}^{3}\right)$ and $s \in \mathbb{R}$,

$$
\left\|(-\Delta+1)^{-1} \int_{\mathbb{R}^{3}} \int_{\mathbb{R}^{3}} e^{-i H_{\varphi_{t}}(k) s} e^{i\left(k+k^{\prime}\right) \cdot x} b_{k^{*}}^{*} b_{k^{\prime}}^{*} u \otimes \Omega \frac{d k^{\prime} d k}{\left|k^{\prime}\right||k|}\right\|_{\mathcal{L}^{2} \otimes \mathcal{F}} \lesssim \alpha^{-2}\|u\|_{\mathcal{H}^{2}} .
$$

Before proving this lemma we show how to use it to bound $Q_{10}$. Note that, since $Q_{10}$ involves only $b_{k}^{*} b_{k^{\prime}}^{*} \Omega$, the operator $(-\Delta+\mathcal{N}+M)^{-1}$ in its definition can be replaced by $\left(-\Delta+2 \alpha^{-2}+M\right)^{-1}$. This observation, together with Lemma 4.1 and the uniform boundedness of $\tilde{\psi}_{s}$ in $\mathcal{H}^{2}$ for $s \in\left[0, \alpha^{2}\right]$ (see Proposition 2.2), proves that

$$
\left\|Q_{10}\right\|_{\mathcal{L}^{2} \otimes \mathcal{F}} \lesssim \alpha^{-2}
$$

Proof of Lemma 4.1. We shall show that for any $\gamma \in \mathcal{L}^{2}\left(\mathbb{R}^{3}\right) \otimes \mathcal{F}$,

$$
\left|\left\langle\gamma,(-\Delta+1)^{-1} \int_{\mathbb{R}^{3}} \int_{\mathbb{R}^{3}} e^{-i H_{\varphi_{t}}(k) s} e^{i\left(k+k^{\prime}\right) \cdot x} b_{k}^{*} b_{k^{\prime}}^{*} u \otimes \Omega \frac{d k^{\prime} d k}{\left|k^{\prime}\right||k|}\right\rangle\right| \lesssim \alpha^{-2}\|\gamma\|_{\mathcal{L}^{2} \otimes \mathcal{F}}\|u\|_{\mathcal{H}^{2}} .
$$

We integrate by parts twice in $x$ and use (3-1) with $k$ replaced by $k+k^{\prime}$. A typical term that is obtained in this way in the inner product on the left side is

$$
\left\langle e^{i H_{\varphi t}(k) s} \partial_{x_{i}} \partial_{x_{j}}(-\Delta+1)^{-1} \gamma, \int_{\mathbb{R}^{3}} \int_{\mathbb{R}^{3}} e^{i\left(k+k^{\prime}\right) \cdot x} b_{k}^{*} b_{k^{\prime}}^{*} u \otimes \Omega \frac{\left(k_{i}+k_{i}^{\prime}\right)\left(k_{j}+k_{j}^{\prime}\right) d k^{\prime} d k}{|k|\left|k^{\prime}\right|\left(1+\left|k+k^{\prime}\right|^{2}\right)^{2}}\right\rangle .
$$

Since $\partial_{x_{i}} \partial_{x_{j}}(-\Delta+1)^{-1}$ is bounded and $e^{i H_{\varphi_{t}}(k) s}$ is unitary, the vector on the left side of the inner product is bounded in norm by $\|\gamma\|_{\mathcal{L}^{2} \otimes \mathcal{F}}$. We now show that the vector on the right side of the inner 
product is bounded as well. We compute

$$
\begin{aligned}
\left\|\int_{\mathbb{R}^{3}} \int_{\mathbb{R}^{3}} e^{i\left(k+k^{\prime}\right) \cdot x} b_{k}^{*} b_{k^{\prime}}^{*} u \otimes \Omega \frac{\left(k_{i}+k_{i}^{\prime}\right)\left(k_{j}+k_{j}^{\prime}\right)}{|k|\left|k^{\prime}\right|\left(1+\left|k+k^{\prime}\right|^{2}\right)^{2}} d k^{\prime} d k\right\|_{\mathcal{L}^{2} \otimes \mathcal{F}}^{2} \\
=2 \alpha^{-4}\|u\|_{2}^{2} \int_{\mathbb{R}^{3}} \int_{\mathbb{R}^{3}} \frac{\left(k_{i}+k_{i}^{\prime}\right)^{2}\left(k_{j}+k_{j}^{\prime}\right)^{2}}{|k|^{2}\left|k^{\prime}\right|^{2}\left(1+\left|k+k^{\prime}\right|^{2}\right)^{4}} d k^{\prime} d k .
\end{aligned}
$$

The desired bound now follows from the fact that the double integral on the right side is finite. Other terms that arise in the integration by parts are controlled similarly and we omit the details.

Bound on $Q_{11}$. By considering the number of involved field particles, we can replace $\mathcal{N}$ in the definition of $Q_{11}$ by numbers and obtain

$$
\begin{aligned}
Q_{11}=\left(-\Delta+\alpha^{-2}+M\right)^{-\frac{1}{2}}\left(\int_{\mathbb{R}^{3}} e^{-i k^{\prime \prime} \cdot x} b_{k^{\prime \prime}}\right. & \left.\frac{d k^{\prime \prime}}{\left|k^{\prime \prime}\right|}\right)\left(-\Delta+2 \alpha^{-2}+M\right)^{-1} \\
& \times \int_{\mathbb{R}^{3}} \int_{\mathbb{R}^{3}} e^{-i H_{\varphi_{t}}(k)(t-s)} e^{i\left(k^{\prime}+k\right) \cdot x} b_{k}^{*} b_{k^{\prime}}^{*} \tilde{\psi}_{s} \otimes \Omega \frac{d k^{\prime}}{\left|k^{\prime}\right|} \frac{d k}{|k|} .
\end{aligned}
$$

Next, by commuting $b_{k^{\prime \prime}}$ to the right,

$$
\begin{aligned}
Q_{11}= & \alpha^{-2}\left(-\Delta+\alpha^{-2}+M\right)^{-\frac{1}{2}} \int_{\mathbb{R}^{3}}((i \nabla \\
& \left.\left.\times k^{\prime}\right)^{2}+2 \alpha^{-2}+M\right)^{-1} \\
& e^{-i k^{\prime} \cdot x} e^{-i H_{\varphi_{t}}(k)(t-s)} e^{i\left(k^{\prime}+k\right) \cdot x} b_{k}^{*} \tilde{\psi}_{s} \otimes \Omega \frac{d k^{\prime}}{\left|k^{\prime}\right|^{2}} \frac{d k}{|k|} \\
+\alpha^{-2}\left(-\Delta+\alpha^{-2}+M\right)^{-\frac{1}{2}} \int_{\mathbb{R}^{3}}((i \nabla & \left.-k)^{2}+2 \alpha^{-2}+M\right)^{-1} \\
& \times \int_{\mathbb{R}^{3}} e^{-i k \cdot x} e^{-i H_{\varphi_{t}}(k)(t-s)} e^{i\left(k^{\prime}+k\right) \cdot x} b_{k^{\prime}}^{*} \tilde{\psi}_{s} \otimes \Omega \frac{d k^{\prime}}{\left|k^{\prime}\right|} \frac{d k}{|k|^{2}} .
\end{aligned}
$$

It remains to compute the norm of this expression. Since this is considerably easier than for $Q_{12}$, we omit the details and only state the final result,

$$
\left\|Q_{11}\right\|_{\mathcal{L}^{2} \otimes \mathcal{F}} \lesssim \alpha^{-3} .
$$

Bound on $Q_{12}$. In the same way as for $Q_{11}$, we can replace $\mathcal{N}$ by a number, so that

$$
\begin{aligned}
Q_{12}=\left(-\Delta+3 \alpha^{-2}+M\right)^{-\frac{1}{2}} \int_{\mathbb{R}^{3}} e^{i k^{\prime \prime} \cdot x} b_{k^{\prime \prime}}^{*} & \left(-\Delta+2 \alpha^{-2}+M\right)^{-1} \\
& \times \int_{\mathbb{R}^{3}} \int_{\mathbb{R}^{3}} e^{-i H_{\varphi_{t}}(k)(t-s)} e^{i\left(k^{\prime}+k\right) \cdot x} b_{k^{\prime}}^{*} b_{k}^{*} \tilde{\psi}_{s} \otimes \Omega \frac{d k^{\prime}}{\left|k^{\prime}\right|} \frac{d k}{|k|} .
\end{aligned}
$$

Next, we commute $e^{i k^{\prime \prime} \cdot x}$ and $e^{i\left(k^{\prime}+k\right) \cdot x}$ to the right and obtain

$$
\begin{aligned}
Q_{12}=\int_{\mathbb{R}^{3}} \int_{\mathbb{R}^{3}} \int_{\mathbb{R}^{3}} b_{k}^{*} b_{k^{\prime}}^{*} b_{k^{\prime \prime}}^{*} e^{i\left(k+k^{\prime}+k^{\prime \prime}\right) \cdot x}\left(\left(i \nabla-k-k^{\prime}-k^{\prime \prime}\right)^{2}+3 \alpha^{-2}+M\right)^{-\frac{1}{2}} \\
\times\left(\left(i \nabla-k-k^{\prime}\right)^{2}+2 \alpha^{-2}+M\right)^{-1} e^{-i H_{\varphi_{t}}\left(-k^{\prime}\right)(t-s)} \tilde{\psi}_{s} \otimes \Omega \frac{d k^{\prime \prime}}{\left|k^{\prime \prime}\right|} \frac{d k^{\prime}}{\left|k^{\prime}\right|} \frac{d k}{|k|} .
\end{aligned}
$$


We now compute the norm of this expression. For the part of the norm over $\mathcal{F}$, we use the fact that

$$
\begin{aligned}
\alpha^{6}\left\langle\Omega, b_{k_{1}} b_{k_{2}} b_{k_{3}} b_{k_{4}}^{*} b_{k_{5}}^{*} b_{k_{6}}^{*} \Omega\right\rangle= & \delta\left(k_{1}-k_{4}\right) \delta\left(k_{2}-k_{5}\right) \delta\left(k_{3}-k_{6}\right)+\delta\left(k_{1}-k_{4}\right) \delta\left(k_{2}-k_{6}\right) \delta\left(k_{3}-k_{5}\right) \\
+ & \delta\left(k_{1}-k_{5}\right) \delta\left(k_{2}-k_{4}\right) \delta\left(k_{3}-k_{6}\right)+\delta\left(k_{1}-k_{5}\right) \delta\left(k_{2}-k_{6}\right) \delta\left(k_{3}-k_{4}\right) \\
& +\delta\left(k_{1}-k_{6}\right) \delta\left(k_{2}-k_{4}\right) \delta\left(k_{3}-k_{5}\right)+\delta\left(k_{1}-k_{6}\right) \delta\left(k_{2}-k_{4}\right) \delta\left(k_{3}-k_{6}\right)
\end{aligned}
$$

to write

$$
\left\|Q_{12}\right\|_{\mathcal{L}^{2} \otimes \mathcal{F}}^{2}=\alpha^{-6}\left(X_{1}+\cdots+X_{6}\right),
$$

where, for instance,

$$
\begin{aligned}
X_{1}:=\int_{\mathbb{R}^{3}} \int_{\mathbb{R}^{3}} \int_{\mathbb{R}^{3}}\left\langle e^{-i H_{\varphi_{t}}\left(-k^{\prime}\right)(t-s)} \tilde{\psi}_{s},\left(\left(i \nabla-k-k^{\prime}-k^{\prime \prime}\right)^{2}+3 \alpha^{-2}+M\right)^{-1}\right. \\
\left.\quad \times\left(\left(i \nabla-k-k^{\prime}\right)^{2}+2 \alpha^{-2}+M\right)^{-2} e^{-i H_{\varphi_{t}}\left(-k^{\prime}\right)(t-s)} \tilde{\psi}_{s}\right\rangle \frac{d k^{\prime \prime}}{\left|k^{\prime \prime}\right|^{2}} \frac{d k^{\prime}}{\left|k^{\prime}\right|^{2}} \frac{d k}{|k|^{2}}
\end{aligned}
$$

and

$$
\begin{aligned}
X_{2} & :=\int_{\mathbb{R}^{3}} \int_{\mathbb{R}^{3}} \int_{\mathbb{R}^{3}}\left\langle e^{-i H_{\varphi_{t}}\left(-k^{\prime \prime}\right)(t-s)} \tilde{\psi}_{s},\left(\left(i \nabla-k-k^{\prime}-k^{\prime \prime}\right)^{2}+3 \alpha^{-2}+M\right)^{-1}\right. \\
& \left.\times\left(\left(i \nabla-k-k^{\prime \prime}\right)^{2}+2 \alpha^{-2}+M\right)^{-1}\left(\left(i \nabla-k-k^{\prime}\right)^{2}+2 \alpha^{-2}+M\right)^{-1} e^{-i H_{\varphi_{t}}\left(-k^{\prime}\right)(t-s)} \tilde{\psi}_{s}\right\rangle \frac{d k^{\prime \prime}}{\left|k^{\prime \prime}\right|^{2}} \frac{d k^{\prime}}{\left|k^{\prime}\right|^{2}} \frac{d k}{|k|^{2}} .
\end{aligned}
$$

By the Schwarz inequality we have $\left|X_{2}\right| \leq X_{1}$ and, similarly,

$$
\left|X_{j}\right| \leq X_{1} \quad \text { for all } j=1, \ldots, 6 .
$$

Thus it suffices to control $X_{1}$.

We first perform the $k^{\prime \prime}$ integral and then the $k$ integral. We make use of the following bounds.

Lemma 4.2. One has the operator inequalities

$$
\begin{gathered}
\int_{\mathbb{R}^{3}}\left(\left(i \nabla-k^{\prime \prime}\right)^{2}+1\right)^{-1} \frac{d k^{\prime \prime}}{\left|k^{\prime \prime}\right|^{2}} \lesssim 1, \\
\int_{\mathbb{R}^{3}}\left(\left(i \nabla_{x}-k\right)^{2}+1\right)^{-2} \frac{d k}{|k|^{2}} \lesssim(-\Delta+1)^{-1} .
\end{gathered}
$$

Before proving the lemma, let us see that they provide the desired bounds on $X_{1}$. First, conjugating (4-10) with $e^{i\left(k+k^{\prime}\right) \cdot x}$ and assuming that $M+3 \alpha^{2} \geq 1$, we obtain, uniformly in $k, k^{\prime} \in \mathbb{R}^{3}$,

$$
\int_{\mathbb{R}^{3}}\left(\left(i \nabla-k-k^{\prime}-k^{\prime \prime}\right)^{2}+3 \alpha^{-2}+M\right)^{-1} \frac{d k^{\prime \prime}}{\left|k^{\prime \prime}\right|^{2}} \lesssim 1 .
$$

Similarly, conjugating (4-11) with $e^{i k^{\prime} \cdot x}$, we obtain, uniformly in $k^{\prime} \in \mathbb{R}^{3}$,

$$
\int_{\mathbb{R}^{3}}\left(\left(i \nabla_{x}-k-k^{\prime}\right)^{2}+2 \alpha^{-2}+M\right)^{-2} \frac{d k}{|k|^{2}} \lesssim\left(\left(i \nabla-k^{\prime}\right)^{2}+1\right)^{-1} .
$$

Inserting (4-12) and (4-13) into the definition of $X_{1}$, we obtain

$$
X_{1} \lesssim \int_{\mathbb{R}^{3}}\left\langle e^{-i H_{\varphi_{t}}\left(-k^{\prime}\right)(t-s)} \tilde{\psi}_{s},\left(\left(i \nabla-k^{\prime}\right)^{2}+1\right)^{-1} e^{-i H_{\varphi_{t}}\left(-k^{\prime}\right)(t-s)} \tilde{\psi}_{s}\right\rangle \frac{d k^{\prime}}{\left|k^{\prime}\right|^{2}} .
$$


Since $(-\Delta+1)^{-\frac{1}{2}}\left(H_{\varphi_{t}}+M\right)^{\frac{1}{2}}$ is bounded, uniformly in $t$ (by Corollary B.2 and Lemma 2.1), we also know that $\left(\left(i \nabla-k^{\prime}\right)^{2}+1\right)^{-\frac{1}{2}}\left(H_{\varphi_{t}}\left(-k^{\prime}\right)+M\right)^{\frac{1}{2}}$ is bounded, uniformly in $t$. Thus,

$$
\begin{aligned}
X_{1} & \lesssim \int_{\mathbb{R}^{3}}\left\langle e^{-i H_{\varphi_{t}}\left(-k^{\prime}\right)(t-s)} \tilde{\psi}_{s},\left(H_{\varphi_{t}}\left(-k^{\prime}\right)+M\right)^{-1} e^{-i H_{\varphi_{t}}\left(-k^{\prime}\right)(t-s)} \tilde{\psi}_{s}\right\rangle \frac{d k^{\prime}}{\left|k^{\prime}\right|^{2}} \\
& =\int_{\mathbb{R}^{3}}\left\langle\tilde{\psi}_{s},\left(H_{\varphi_{t}}\left(-k^{\prime}\right)+M\right)^{-1} \tilde{\psi}_{s}\right\rangle \frac{d k^{\prime}}{\left|k^{\prime}\right|^{2}} \\
& \lesssim \int_{\mathbb{R}^{3}}\left\langle\tilde{\psi}_{s},\left(\left(i \nabla-k^{\prime}\right)^{2}+M\right)^{-1} \tilde{\psi}_{s}\right\rangle \frac{d k^{\prime}}{\left|k^{\prime}\right|^{2}} .
\end{aligned}
$$

Applying (4-10) again, we see that the latter expression is bounded by a constant times $\left\|\tilde{\psi}_{s}\right\|_{\mathcal{L}^{2}}^{2}=1$ by Lemma 2.1. This, together with (4-8) and (4-9), implies that

$$
\left\|Q_{12}\right\|_{\mathcal{L}^{2} \otimes \mathcal{F}} \lesssim \alpha^{-3} .
$$

Proof of Lemma 4.2. We only prove (4-11), since the proof of (4-10) is similar and simpler. By applying a Fourier transform, we see that we need to prove

$$
\int_{\mathbb{R}^{3}}\left((p+k)^{2}+1\right)^{-2} \frac{d k}{|k|^{2}} \lesssim\left(p^{2}+1\right)^{-1} \text { for } p \in \mathbb{R}^{3} .
$$

We split the integral into the regions $4|k|>|p|+1$ and $4|k| \leq|p|+1$. In the first region we bound $|k|^{-2} \leq 16 /(|p|+1)^{2}$ and note that

$$
\int_{\{4|k|>|p|+1\}}\left((p+k)^{2}+1\right)^{-2} d k \leq \int_{\mathbb{R}^{3}}\left((p+k)^{2}+1\right)^{-2} d k=\int_{\mathbb{R}^{3}}\left(k^{2}+1\right)^{-2} d k<\infty .
$$

In the second region we distinguish the cases $|p|<1$ and $|p| \geq 1$. In the first case we bound

$$
\int_{\{4|k| \leq|p|+1\}}\left((p+k)^{2}+1\right)^{-2} \frac{d k}{|k|^{2}} \leq \int_{\{4|k| \leq|p|+1\}} \frac{d k}{|k|^{2}} \leq \int_{\left\{|k| \leq \frac{1}{2}\right\}} \frac{d k}{|k|^{2}}<\infty .
$$

For $|p| \geq 1$ we note that in the second region we have $2|k| \leq|p|$ and therefore $(p+k)^{2} \geq \frac{1}{4} p^{2} \geq k^{2}$. Thus,

$$
\left((p+k)^{2}+1\right)^{-2} \leq\left(\frac{1}{4} p^{2}+1\right)^{-1}\left(k^{2}+1\right)^{-1} .
$$

Since $\left(k^{2}+1\right)^{-1}|k|^{-2}$ is integrable, we obtain again a bound of the required form.

Bounds on $Q_{2}, \ldots, Q_{5}$. The terms $Q_{2}, \ldots, Q_{4}$ are controlled in exactly the same way as $Q_{1}$. (For $Q_{4}$ we use the fact that $\left\|\partial_{s} \tilde{\psi}_{s}\right\|_{\mathcal{H}^{2}} \lesssim 1$ for $t \leq \alpha^{2}$ by Proposition 2.2.) The argument for $Q_{5}$ is also similar. In fact, the term involving $\operatorname{Im}\left(\varphi_{s}, \alpha^{2} \partial_{s} \varphi_{s}\right)$ is controlled as before. For the term involving $b^{*}\left(\alpha^{2} \partial_{s} \varphi_{s}\right)$ we have to prove a simple extension of Lemma 4.1 where we have operators $b^{*}(f) b_{k}^{*} b_{k^{\prime}}^{*}$ with $f \in \mathcal{L}^{2}$ (similarly as the second part in Lemma 3.1). Finally, the term involving $b\left(\alpha^{2} \partial_{s} \varphi_{s}\right)$ can be commuted to the right and therefore becomes a less singular term which can be controlled already with Lemma 3.1. These arguments prove (4-2) and complete the proof of (4-3). 
Bound on $\boldsymbol{D}_{112}$. The term $D_{112}$ in (2-34) contains only one factor $\left|k^{\prime}\right|^{-1}$ and can therefore be controlled essentially by the same method as $D_{01}$, based on Lemma 3.1. In order to create a factor of $\left(H_{\varphi_{t}}+M\right)^{-1}$, we integrate by parts in $s_{1}$. This, however, will create a factor of $\tilde{H}_{\varphi_{t}}$ in one of the terms. When dealing with $D_{211}$ we will explain how to remove this term by integrating by parts in $s$. Since $\left\|g_{s, t}\right\|_{\infty} \lesssim \alpha^{-2}|t-s|$ and $\left\|\partial_{s} g_{s, t}\right\|_{\infty}=\left\|g_{s}\right\|_{\infty} \lesssim \alpha^{-2}$ by Proposition 2.2, this factor behaves well in the bounds. When applying Lemma 3.1 we also use $\left\|\partial_{s} \tilde{\psi}_{s}\right\|_{\mathcal{H}^{1}} \lesssim 1$ from Proposition 2.2; see also the remark at the beginning of Section $2 \mathrm{~B}$ concerning the bounds on $\partial_{t} \tilde{\psi}_{t}$. Without going into details we state the final result,

$$
\left\|D_{112}\right\|_{\mathcal{L}^{2} \otimes \mathcal{F}} \lesssim \alpha^{-3} t^{2}(1+t)
$$

Bound on $\boldsymbol{D}_{121}$. Also the term $D_{121}$ in (2-31) contains only one factor of $|k|^{-1}$ and can be controlled as just sketched for $D_{112}$ and as explained in detail for $D_{211}$. In order to control the terms that appear when integrating by parts in $s$ we make use of $\left\|\partial_{s} \sigma_{\tilde{\psi}_{s}}\right\|_{\mathcal{L}^{2}} \lesssim 1$ and $\left\|\partial_{s} \tilde{\psi}_{s}\right\|_{\mathcal{H}^{1}} \lesssim 1$ from Proposition 2.2 in addition to the bounds from Lemma 2.1. Moreover, we need an obvious extension of Lemma 3.1 to the case with $b^{*}\left(f_{1}\right) b^{*}\left(f_{2}\right) b_{k}^{*}$, which is proved in the same way. Combining all this, we end up with

$$
\left\|D_{121}\right\|_{\mathcal{L}^{2} \otimes \mathcal{F}} \lesssim \alpha^{-2} t(1+t) .
$$

Bound on $D_{122}$. The term $D_{122}$ contains no $|k|^{-1}$ term. Using $\left\|g_{s, t}\right\|_{\infty} \lesssim \alpha^{-2}|t-s|$ for $0 \leq s \leq t \leq \alpha^{2}$ by Proposition 2.2 and $\left\|b\left(\sigma_{\tilde{\psi}_{s}}\right) \Omega\right\|_{\mathcal{F}}=\alpha^{-1}\left\|\sigma_{\tilde{\psi}_{s}}\right\|_{2} \lesssim \alpha^{-1}$ by Lemma 2.1, we obtain immediately

$$
\left\|D_{122}\right\|_{\mathcal{L}^{2} \otimes \mathcal{F}} \lesssim \alpha^{-3} t^{3}
$$

\section{Estimation on $D_{2}$}

Bound on $\boldsymbol{D}_{211}$. We recall equation (2-32) for $D_{211}$. In this equation we commute $e^{-i k \cdot x}$ through $e^{-i H_{\varphi_{t}} s_{1}}$, which introduces again the operator $H_{\varphi_{t}}(k)$ from (4-1), and we commute $b_{k}$ with $b_{k^{\prime}}^{*}$. In this way, we obtain

$$
D_{211}=\alpha^{-2} \int_{0}^{t} \int_{0}^{t-s} \int_{\mathbb{R}^{3}} e^{i \tilde{H}_{\varphi_{t}}\left(s+s_{1}\right)} e^{-i H_{\varphi_{t}}(k) s_{1}} W^{*}\left(\alpha^{2} \varphi_{t}\right) W\left(\alpha^{2} \varphi_{s}\right) \tilde{\psi}_{s} \otimes \Omega \frac{d k}{|k|^{2}} d s_{1} d s .
$$

The difficulty in controlling $D_{211}$ comes again from the $k$-integral. It is not enough to bound the norm of the integrand as it stands, since $|k|^{-2}$ is not integrable. Thus, we need to gain some extra decay from $e^{-i H_{\varphi_{t}}(k) s_{1}}$. To get this decay, we integrate by parts in $s_{1}$ using

$$
e^{-i H_{\varphi_{t}}(k) s_{1}}=i e^{i M s_{1}}\left(H_{\varphi_{t}}(k)+M\right)^{-1} \partial_{s_{1}} e^{-i\left[H_{\varphi_{t}}(k)+M\right] s_{1}}
$$

with a large constant $M>0$ independent of $\alpha$ and $t$. We obtain

$$
\begin{gathered}
D_{211}=i \alpha^{-2} \int_{0}^{t} \int_{\mathbb{R}^{3}} e^{i \tilde{H}_{\varphi_{t}} t}\left(H_{\varphi_{t}}(k)+M\right)^{-1} e^{-i H_{\varphi_{t}}(k)(t-s)} W^{*}\left(\alpha^{2} \varphi_{t}\right) W\left(\alpha^{2} \varphi_{s}\right) \tilde{\psi}_{s} \otimes \Omega \frac{d k}{|k|^{2}} d s \\
-i \alpha^{-2} \int_{0}^{t} \int_{\mathbb{R}^{3}} e^{i \tilde{H}_{\varphi_{t}} s}\left(H_{\varphi_{t}}(k)+M\right)^{-1} W^{*}\left(\alpha^{2} \varphi_{t}\right) W\left(\alpha^{2} \varphi_{s}\right) \tilde{\psi}_{s} \otimes \Omega \frac{d k}{|k|^{2}} d s
\end{gathered}
$$




$$
\begin{gathered}
+\alpha^{-2} M \int_{0}^{t} \int_{0}^{t-s} \int_{\mathbb{R}^{3}} e^{i \tilde{H}_{\varphi_{t}}\left(s+s_{1}\right)}\left(H_{\varphi_{t}}(k)+M\right)^{-1} e^{-i H_{\varphi_{t}}(k) s_{1}} \\
\times W^{*}\left(\alpha^{2} \varphi_{t}\right) W\left(\alpha^{2} \varphi_{s}\right) \tilde{\psi}_{s} \otimes \Omega \frac{d k}{|k|^{2} d s_{1} d s} \\
+\alpha^{-2} \int_{0}^{t} \int_{0}^{t-s} \int_{\mathbb{R}^{3}} e^{i \tilde{H}_{\varphi_{t}}\left(s+s_{1}\right)} \tilde{H}_{\varphi_{t}}\left(H_{\varphi_{t}}(k)+M\right)^{-1} e^{-i H_{\varphi_{t}}(k) s_{1}} \\
\times W^{*}\left(\alpha^{2} \varphi_{t}\right) W\left(\alpha^{2} \varphi_{s}\right) \tilde{\psi}_{s} \otimes \Omega \frac{d k}{|k|^{2}} d s_{1} d s \\
=D_{2111}+D_{2112}+D_{2113}+D_{2114},
\end{gathered}
$$

where $D_{211 k}, k=1, \ldots, 4$, are naturally defined.

We first show how to deal with the terms $D_{2111}, D_{2112}$ and $D_{2113}$. The term $D_{2114}$ is harder because of the additional factor of $\tilde{H}_{\varphi_{t}}$.

The following lemma quantifies in which sense the operator $\left(H_{\varphi_{t}}+M\right)^{-1}$ leads to additional decay in $k$.

Lemma 5.1. For $u \in \mathcal{H}^{2}\left(\mathbb{R}^{3}\right)$,

$$
\int_{\mathbb{R}^{3}}\left\|\left(|i \nabla+k|^{2}+1\right)^{-1} u\right\|_{2} \frac{d k}{|k|^{2}} \lesssim\|u\|_{\mathcal{H}^{2}}
$$

Proof. By Fourier transform, we have

$$
\left\|\left(|i \nabla+k|^{2}+1\right)^{-1} u\right\|_{2}^{2}=\int_{\mathbb{R}^{3}} \frac{1}{\left(1+|p+k|^{2}\right)^{2}\left(1+|p|^{2}\right)^{2}}\left(1+|p|^{2}\right)^{2}|\hat{u}(p)|^{2} d p .
$$

We now observe that

$$
\frac{1}{\left(1+|p+k|^{2}\right)^{2}\left(1+|p|^{2}\right)^{2}} \lesssim \frac{1}{\left(1+|k|^{2}\right)^{2}}
$$

This can be proved by considering separately the regions where $|p| \leq \frac{1}{2}|k|$ and $|p| \geq \frac{1}{2}|k|$. Thus,

$$
\left\|\left(|i \nabla+k|^{2}+1\right)^{-1} u\right\|_{2}^{2} \lesssim \frac{1}{\left(1+|k|^{2}\right)^{2}}\|u\|_{\mathcal{H}^{2}}^{2},
$$

and the claimed bound follows by integration over $k$.

Let us return to the terms $D_{2111}, D_{2112}$ and $D_{2113}$. It follows from Corollary B.2 by conjugating with the unitary $e^{i k \cdot x}$ that there is an $M>0$ such that the operator $\left(H_{\varphi_{t}}(k)+M\right)^{-1}\left(|i \nabla+k|^{2}+1\right)$ is uniformly bounded in $\alpha$ and $t$. This, together with the boundedness of $\psi_{s}$ in $\mathcal{H}^{2}$ for $s \in\left[0, \alpha^{2}\right]$ from Proposition 2.2, yields

$$
\int_{\mathbb{R}^{3}}\left\|\left(H_{\varphi_{t}}(k)+M\right)^{-1} \tilde{\psi}_{s}\right\|_{2} \frac{d k}{|k|^{2}} \lesssim 1
$$

and therefore

$$
\left\|D_{2111}\right\|_{\mathcal{L}^{2} \otimes \mathcal{F}} \lesssim \alpha^{-2} t, \quad\left\|D_{2112}\right\|_{\mathcal{L}^{2} \otimes \mathcal{F}} \lesssim \alpha^{-2} t, \quad\left\|D_{2113}\right\|_{\mathcal{L}^{2} \otimes \mathcal{F}} \lesssim \alpha^{-2} t^{2}
$$


We now turn to the term $D_{2114}$, which contains the operator $\tilde{H}_{\varphi_{t}}$. The idea is to remove this operator by integrating by parts in $s$ using

$$
\tilde{H}_{\varphi_{t}} e^{i \tilde{H}_{\varphi_{t}} s}=-i \partial_{s} e^{i \tilde{H}_{\varphi_{t}} s}
$$

This leads to

$$
\begin{gathered}
D_{2114}=-i \alpha^{-2} \int_{0}^{t} \int_{\mathbb{R}^{3}} e^{i \tilde{H}_{\varphi_{t}} t}\left(H_{\varphi_{t}}(k)+M\right)^{-1} e^{-i H_{\varphi_{t}}(k)\left(t-s_{1}\right)} W^{*}\left(\alpha^{2} \varphi_{t}\right) W\left(\alpha^{2} \varphi_{s_{1}}\right) \tilde{\psi}_{s_{1}} \otimes \Omega \frac{d k}{|k|^{2}} d s_{1} \\
+i \alpha^{-2} \int_{0}^{t} \int_{\mathbb{R}^{3}} e^{i \tilde{H}_{\varphi_{t}} s_{1}}\left(H_{\varphi_{t}}(k)+M\right)^{-1} e^{-i H_{\varphi_{t}}(k) s_{1}} W^{*}\left(\alpha^{2} \varphi_{t}\right) W\left(\alpha^{2} \varphi_{0}\right) \tilde{\psi}_{0} \otimes \Omega \frac{d k}{|k|^{2}} d s_{1} \\
+i \alpha^{-2} \int_{0}^{t} \int_{0}^{t-s} \int_{\mathbb{R}^{3}} e^{i \widetilde{H}_{\varphi_{t}}\left(s+s_{1}\right)}\left(H_{\varphi_{t}}(k)+M\right)^{-1} e^{-i H_{\varphi_{t}}(k) s_{1}} \\
\times W^{*}\left(\alpha^{2} \varphi_{t}\right) W\left(\alpha^{2} \varphi_{s}\right) \partial_{s} \tilde{\psi}_{s} \otimes \Omega \frac{d k}{|k|^{2}} d s_{1} d s \\
+i \alpha^{-2} \int_{0}^{t} \int_{0}^{t-s} \int_{\mathbb{R}^{3}} e^{i \tilde{H}_{\varphi_{t}}\left(s+s_{1}\right)}\left(H_{\varphi_{t}}(k)+M\right)^{-1} e^{-i H_{\varphi_{t}}(k) s_{1}} \\
\times W^{*}\left(\alpha^{2} \varphi_{t}\right)\left(\partial_{s} W\left(\alpha^{2} \varphi_{s}\right)\right) \tilde{\psi}_{s} \otimes \Omega \frac{d k}{|k|^{2}} d s_{1} d s
\end{gathered}
$$

The first three terms on the right side can be bounded by Lemma 5.1 together with the uniform boundedness in $\mathcal{H}^{2}$ of $\tilde{\psi}_{s}$ and $\partial_{s} \tilde{\psi}_{s}$ in $\left[0, \alpha^{2}\right]$ from Proposition 2.2; see also the remark at the beginning of Section $2 \mathrm{~B}$ concerning the bounds on $\partial_{t} \tilde{\psi}_{t}$. For the fourth term on the right side we use the formula (A-4) for $\partial_{s} W\left(\alpha^{2} \varphi_{s}\right)$. Then the term can be bounded by proceeding in the same way as for $D_{015}$ and using Lemma 5.1 together with the fact that $\alpha^{2} \partial_{s} \varphi_{s}$ is uniformly bounded in $\mathcal{L}^{2}$ for all times by Lemma 2.1. To summarize, we obtain

$$
\left\|D_{2114}\right\|_{\mathcal{L}^{2} \otimes \mathcal{F}} \lesssim \alpha^{-2} t(1+t)
$$

and, because of (5-3),

$$
\left\|D_{211}\right\|_{\mathcal{L}^{2} \otimes \mathcal{F}} \lesssim \alpha^{-2} t(1+t)
$$

Bound on $D_{212}$. The term $D_{212}$ involves a single difficult operator $\int b_{k^{\prime}}^{*} e^{i k^{\prime} \cdot x}\left|k^{\prime}\right|^{-1} d k^{\prime}$ and can be controlled using the technique from bounding $D_{01}$. We first integrate by parts with respect to $s_{1}$ using (5-1) (with $k=0$ ) to create a factor of $\left(H_{\varphi_{t}}+M\right)^{-1}$. Using this factor we can apply Lemma 3.1 as in the bound of $D_{01}$. In one of the terms, however, the integration by parts creates a factor $\tilde{H}_{\varphi_{t}}$. We remove this operator via (5-4) by integrating by parts in $s$. The factor $g_{s, t}$ and its derivative $\partial_{s} g_{s, t}=-g_{s}$ are bounded by Proposition 2.2 and do not create any problems. Eventually, this shows that

$$
\left\|D_{212}\right\|_{\mathcal{L}^{2} \otimes \mathcal{F}} \lesssim \alpha^{-3} t^{2}(1+t)
$$

Bound on $D_{221}$. The term $D_{221}$ appears in (2-33). We use $b_{k} b^{*}\left(\sigma_{\tilde{\psi}_{s}}\right) \Omega=\alpha^{-2} \sigma_{\tilde{\psi}_{s}}(k) \Omega$. By the Schwarz inequality, (C-2) and Lemma 2.1 we have $\left\||k|^{-1} \sigma_{\tilde{\psi}_{s}}(k) \Omega\right\|_{1} \lesssim\left\|\sigma_{\tilde{\psi}_{s}}\right\|_{\mathcal{L}_{(1)}^{2}} \lesssim\left\|\psi_{s}\right\|_{H^{1}}^{2} \lesssim 1$. From this one easily concludes that

$$
\left\|D_{221}\right\|_{\mathcal{L}^{2} \otimes \mathcal{F}} \lesssim \alpha^{-2} t^{2} .
$$


Bound on $\boldsymbol{D}_{\mathbf{2 2 2}}$. The term $D_{222}$ appears in (2-37). Using the bound on $g_{s, t}$ from Proposition 2.2 and the fact that $b\left(\sigma_{\tilde{\psi}_{s}}\right) \Omega$ has norm of order $\alpha^{-1}$ by Lemma 2.1, one obtains

$$
\left\|D_{222}\right\|_{\mathcal{L}^{2} \otimes \mathcal{F}} \lesssim \alpha^{-3} t^{3}
$$

\section{Bounds on $D_{3}, D_{4}$ and $D_{5}$}

We recall that we have already controlled $D_{32}, D_{42}$ and $D_{52}$ in (2-39), (2-40) and (2-42). The remaining terms $D_{31}, D_{41}$ and $D_{51}$ have at most a single term $|k|^{-1}$ and can be bounded using the methods we have already developed. Therefore we will be rather brief.

For each of the terms $D_{311}, D_{312}, D_{412}, D_{511}$ and $D_{512}$ we first integrate by parts in $s_{1}$ to generate a factor of $\left(H_{\varphi_{t}}+M\right)^{-1}$, which allows us to apply Lemma 3.1. One of the terms, however, will involve $\widetilde{H}_{\varphi_{t}}$, which we have to remove by integrating by parts in $s$. Using the bounds from Lemma 2.1 and Proposition 2.2 we obtain

$$
\begin{gathered}
\left\|D_{311}\right\|_{\mathcal{L}^{2} \otimes \mathcal{F}} \lesssim \alpha^{-2} t(1+t), \quad\left\|D_{312}\right\|_{\mathcal{L}^{2} \otimes \mathcal{F}} \lesssim \alpha^{-3} t^{2}(1+t), \quad\left\|D_{412}\right\|_{\mathcal{L}^{2} \otimes \mathcal{F}} \lesssim \alpha^{-3} t^{2}(1+t) \\
\left\|D_{511}\right\|_{\mathcal{L}^{2} \otimes \mathcal{F}} \lesssim \alpha^{-3} t(1+t), \quad\left\|D_{512}\right\|_{\mathcal{L}^{2} \otimes \mathcal{F}} \lesssim \alpha^{-4} t^{2}\left(1+t+\alpha^{-1} t^{2}\right)
\end{gathered}
$$

The remaining term $D_{411}$ can be immediately bounded by

$$
\left\|D_{411}\right\|_{\mathcal{L}^{2} \otimes \mathcal{F}} \lesssim \alpha^{-2} t^{2}
$$

\section{Proof of the almost orthogonality relations}

7A. Proof of (2-28). We recall that

$$
\left\langle\Omega, e^{-i H_{\varphi_{t}} t} D_{0}\right\rangle_{\mathcal{F}}=\left\langle\Omega, \int_{0}^{t} e^{-i H_{\varphi_{t}}(t-s)} P_{\tilde{\psi}_{s}}^{\perp} \int_{\mathbb{R}^{3}}\left(e^{i k \cdot x} W^{*}\left(\alpha^{2} \varphi_{t}\right) W\left(\alpha^{2} \varphi_{s}\right) b_{k}^{*} \tilde{\psi}_{s} \otimes \Omega\right) \frac{d k}{|k|} d s\right\rangle_{\mathcal{F}} .
$$

We commute the operator $b_{k}^{*}$ to the left and use $b_{k} \Omega=0$. For the commutator we obtain from Corollary A.2 (with the definition (2-5) of $g_{s, t}$ )

$$
\begin{aligned}
\left\langle\Omega, e^{-i H_{\varphi_{t}} t} D_{0}\right\rangle_{\mathcal{F}} & =\left\langle\Omega, \int_{0}^{t} e^{-i H_{\varphi_{t}}(t-s)} P_{\tilde{\psi}_{s}}^{\perp} g_{s, t} W^{*}\left(\alpha^{2} \varphi_{t}\right) W\left(\alpha^{2} \varphi_{s}\right) \tilde{\psi}_{s} \otimes \Omega d s\right\rangle_{\mathcal{F}} \\
& =\int_{0}^{t} e^{-i H_{\varphi_{t}}(t-s)} P_{\tilde{\psi}_{s}}^{\perp} g_{s, t} \tilde{\psi}_{s}\left\langle\Omega, W^{*}\left(\alpha^{2} \varphi_{t}\right) W\left(\alpha^{2} \varphi_{s}\right) \Omega\right\rangle_{\mathcal{F}} d s
\end{aligned}
$$

Thus,

$$
\left\|\left\langle\Omega, e^{-i H_{\varphi_{t}} t} D_{0}\right\rangle_{\mathcal{F}}\right\|_{\mathcal{L}^{2}} \leq t \sup _{0 \leq s \leq t}\left\|g_{s, t}\right\|_{\infty}\left\|\tilde{\psi}_{s}\right\|_{2}
$$

Thus, by the bound on $g_{s, t}$ from Proposition 2.2 and the conservation of the $\mathcal{L}^{2}$ norm of $\tilde{\psi}_{s}$, we obtain the claimed bound (2-28). 
7B. Proof of (2-29). For $\Phi \in \mathcal{F}$, let

$$
\begin{aligned}
\Theta_{\Phi}(t) & :=\left\langle\tilde{\psi}_{t} \otimes \Phi, e^{-i H_{\varphi_{t}} t} D_{0}\right\rangle_{\mathcal{L}^{2} \otimes \mathcal{F}} \\
& =\left\langle\tilde{\psi}_{t} \otimes \Phi, \int_{0}^{t} e^{-i H_{\varphi_{t}}(t-s)} P_{\tilde{\psi}_{s}}^{\perp} \int_{\mathbb{R}^{3}}\left(e^{i k \cdot x} W^{*}\left(\alpha^{2} \varphi_{t}\right) W\left(\alpha^{2} \varphi_{s}\right) b_{k}^{*} \tilde{\psi}_{s} \otimes \Omega\right) \frac{d k}{|k|} d s\right\rangle_{\mathcal{L}^{2} \otimes \mathcal{F}} .
\end{aligned}
$$

We shall show that

$$
\left|\Theta_{\Phi}(t)\right| \lesssim \alpha^{-2} t^{2}\left(1+\alpha^{-2} t^{2}\right)\|\Phi\|_{\mathcal{F}}
$$

which by duality implies (2-29).

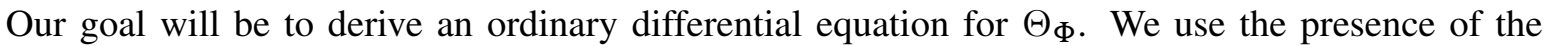
operator $P \underset{\tilde{\psi}_{s}}{\perp}$ to obtain (with inner products in $\mathcal{L}^{2} \otimes \mathcal{F}$ )

$$
\begin{aligned}
\partial_{t} \Theta_{\Phi}= & \left\langle\partial_{t} \tilde{\psi}_{t} \otimes \Phi, \int_{0}^{t} e^{-i H_{\varphi_{t}}(t-s)} P_{\tilde{\psi}_{s}}^{\perp} \int_{\mathbb{R}^{3}}\left(e^{i k \cdot x} W^{*}\left(\alpha^{2} \varphi_{t}\right) W\left(\alpha^{2} \varphi_{s}\right) b_{k}^{*} \tilde{\psi}_{s} \otimes \Omega\right) \frac{d k}{|k|} d s\right\rangle \\
& +\left\langle\tilde{\psi}_{t} \otimes \Phi, \int_{0}^{t}\left(\partial_{t} e^{-i H_{\varphi_{t}}(t-s)}\right) P_{\tilde{\psi}_{s}}^{\perp} \int_{\mathbb{R}^{3}}\left(e^{i k \cdot x} W^{*}\left(\alpha^{2} \varphi_{t}\right) W\left(\alpha^{2} \varphi_{s}\right) b_{k}^{*} \tilde{\psi}_{s} \otimes \Omega\right) \frac{d k}{|k|} d s\right\rangle \\
& +\left\langle\tilde{\psi}_{t} \otimes \Phi, \int_{0}^{t} e^{-i H_{\varphi_{t}}(t-s)} P_{\tilde{\psi}_{s}}^{\perp} \int_{\mathbb{R}^{3}}\left(e^{i k \cdot x}\left(\partial_{t} W^{*}\left(\alpha^{2} \varphi_{t}\right)\right) W\left(\alpha^{2} \varphi_{s}\right) b_{k}^{*} \tilde{\psi}_{s} \otimes \Omega\right) \frac{d k}{|k|} d s\right\rangle .
\end{aligned}
$$

For the first term we use equation (2-10) for $\partial_{t} \tilde{\psi}_{t}$. In the second term, we compute, using Duhamel's formula,

$$
\begin{aligned}
\partial_{t} e^{-i H_{\varphi_{t}}(t-s)} & =-i H_{\varphi_{t}} e^{-i H_{\varphi_{t}}(t-s)}-i \int_{0}^{t-s} e^{-i H_{\varphi_{t}}\left(t-s-s_{1}\right)}\left(\partial_{t} H_{\varphi_{t}}\right) e^{-i H_{\varphi_{t}} s_{1}} d s_{1} \\
& =-i\left(H_{\varphi_{t}}+(t-s) \partial_{t}\left\|\varphi_{t}\right\|_{2}^{2}\right) e^{-i H_{\varphi_{t}}(t-s)}-i \int_{0}^{t-s} e^{-i H_{\varphi_{t}}\left(t-s-s_{1}\right)}\left(\partial_{t} V_{\varphi_{t}}\right) e^{-i H_{\varphi_{t}} s_{1}} d s_{1}
\end{aligned}
$$

Note that the part involving $H_{\varphi_{t}}$ will cancel the contribution from the first term, except for part of the constant $\omega(t)$. Finally, for the third term we use Lemma A.3 and Lemma A.1 to obtain

$$
\begin{aligned}
\partial_{t} W^{*}\left(\alpha^{2} \varphi_{t}\right) & W\left(\alpha^{2} \varphi_{s}\right) \\
= & \alpha^{2} W^{*}\left(\alpha^{2} \varphi_{t}\right)\left[b\left(\partial_{t} \varphi_{t}\right)-b^{*}\left(\partial_{t} \varphi_{t}\right)+i \operatorname{Im}\left(\varphi_{t}, \partial_{t} \varphi_{t}\right)\right] W\left(\alpha^{2} \varphi_{s}\right) \\
= & \alpha^{2} W^{*}\left(\alpha^{2} \varphi_{t}\right) W\left(\alpha^{2} \varphi_{s}\right)\left[b\left(\partial_{t} \varphi_{t}\right)-b^{*}\left(\partial_{t} \varphi_{t}\right)+2 i \operatorname{Im}\left(\partial_{t} \varphi_{t}, \varphi_{s}\right)+i \operatorname{Im}\left(\varphi_{t}, \partial_{t} \varphi_{t}\right)\right] \\
= & \alpha^{2} W^{*}\left(\alpha^{2} \varphi_{t}\right) W\left(\alpha^{2} \varphi_{s}\right)\left[b\left(\partial_{t} \varphi_{t}\right)-b^{*}\left(\partial_{t} \varphi_{t}\right)+2 i \operatorname{Im}\left(\partial_{t} \varphi_{t}, \varphi_{s}-\varphi_{t}\right)+i \operatorname{Im}\left(\partial_{t} \varphi_{t}, \varphi_{t}\right)\right]
\end{aligned}
$$

Putting all this into the above formula, we obtain

$$
\partial_{t} \Theta_{\Phi}=M_{1}+M_{2}+M_{3}
$$

where the terms $M_{1}, M_{2}$ and $M_{3}$ are defined, using the notation

$$
\Phi_{s, t}:=W^{*}\left(\alpha^{2} \varphi_{s}\right) W\left(\alpha^{2} \varphi_{t}\right) \Phi,
$$


by

$$
\begin{aligned}
& M_{1}(t):=-i \int_{0}^{t} \int_{0}^{t-s}\left\langle\tilde{\psi}_{t} \otimes \Phi_{s, t}, e^{-i H_{\varphi_{t}}\left(t-s-s_{1}\right)}\left(\partial_{t} V_{\varphi_{t}}\right) e^{-i H_{\varphi_{t}} s_{1}} P_{\tilde{\psi}_{s}}^{\perp} \int_{\mathbb{R}^{3}}\left(e^{i k \cdot x} b_{k}^{*} \tilde{\psi}_{s} \otimes \Omega\right) \frac{d k}{|k|}\right\rangle d s_{1} d s, \\
& M_{2}(t):=\alpha^{2} \int_{0}^{t}\left\langle\tilde{\psi}_{t} \otimes \Phi_{s, t}, e^{-i H_{\varphi_{t}}(t-s)} P_{\tilde{\psi}_{s}}^{\perp} \int_{\mathbb{R}^{3}}\left(e^{i k \cdot x}\left(b\left(\partial_{t} \varphi_{t}\right)-b^{*}\left(\partial_{t} \varphi_{t}\right)\right) b_{k}^{*} \tilde{\psi}_{s} \otimes \Omega\right) \frac{d k}{|k|}\right\rangle d s, \\
& M_{3}(t):=\int_{0}^{t} m(s, t)\left\langle\tilde{\psi}_{t} \otimes \Phi_{s, t}, e^{-i H_{\varphi_{t}}(t-s)} P_{\tilde{\psi}_{s}}^{\perp} \int_{\mathbb{R}^{3}}\left(e^{i k \cdot x} b_{k}^{*} \tilde{\psi}_{s} \otimes \Omega\right) \frac{d k}{|k|}\right\rangle d s
\end{aligned}
$$

with

$$
m(s, t):=-i(t-s) \partial_{t}\left\|\varphi_{t}\right\|_{2}^{2}+2 i \alpha^{2} \operatorname{Im}\left(\partial_{t} \varphi_{t}, \varphi_{s}-\varphi_{t}\right)
$$

Since $\Theta_{\Phi}(0)=0$, we conclude that

$$
\Theta_{\Phi}(t)=\int_{0}^{t}\left(M_{1}(s)+M_{2}(s)+M_{3}(s)\right) d s .
$$

Below we shall show that

$$
\left|M_{1}(t)\right| \lesssim \alpha^{-3} t^{2}\|\Phi\|_{\mathcal{F}}, \quad\left|M_{2}(t)\right| \lesssim \alpha^{-2} t\|\Phi\|_{\mathcal{F}}, \quad\left|M_{3}(t)\right| \lesssim \alpha^{-3} t^{2}\|\Phi\|_{\mathcal{F}}
$$

Together with (7-2) this will prove (7-1) and therefore (2-29).

Bound on $M_{1}$. Using the fact that $P_{\tilde{\psi}_{s}}^{\perp}=1-\left|\tilde{\psi}_{s}\right\rangle\left\langle\tilde{\psi}_{s}\right|$ (see the proof of Lemma 2.4), we have the decomposition

$$
M_{1}=M_{11}-M_{12}
$$

where

$M_{11}(t):=-i \int_{0}^{t} \int_{0}^{t-s}\left\langle\tilde{\psi}_{t} \otimes \Phi_{s, t}, e^{-i H_{\varphi_{t}}\left(t-s-s_{1}\right)}\left(\partial_{t} V_{\varphi_{t}}\right) e^{-i H_{\varphi_{t}} s_{1}} \int_{\mathbb{R}^{3}}\left(e^{i k \cdot x} b_{k}^{*} \tilde{\psi}_{s} \otimes \Omega\right) \frac{d k}{|k|}\right\rangle_{\mathcal{L}^{2} \otimes \mathcal{F}} d s_{1} d s$ and, with $\sigma_{\tilde{\psi}_{s}}$ from $(2-2)$,

$$
M_{12}(t):=-i \int_{0}^{t} \int_{0}^{t-s}\left\langle\tilde{\psi}_{t}, e^{-i H_{\varphi_{t}}\left(t-s-s_{1}\right)}\left(\partial_{t} V_{\varphi_{t}}\right) e^{-i H_{\varphi_{t}} s_{1}} \tilde{\psi}_{s}\right\rangle_{\mathcal{L}^{2}}\left\langle\Phi_{s, t}, b^{*}\left(\sigma_{\tilde{\psi}_{s}}\right) \Omega\right\rangle_{\mathcal{F}} d s_{1} d s
$$

The second term is easy to control. In fact, the a priori bounds from Lemma 2.1 together with $\left\|\partial_{t} V_{\varphi_{t}}\right\|_{\infty} \lesssim$ $\alpha^{-2}$ from (C-8) imply

$$
\left|\left\langle\tilde{\psi}_{t}, e^{-i H_{\varphi_{t}}\left(t-s-s_{1}\right)}\left(\partial_{t} V_{\varphi_{t}}\right) e^{-i H_{\varphi_{t}} s_{1}} \tilde{\psi}_{s}\right\rangle_{\mathcal{L}^{2}}\right| \lesssim \alpha^{-2}
$$

and

$$
\left|\left\langle\Phi_{s, t}, b^{*}\left(\sigma_{\widetilde{\psi}_{s}}\right) \Omega\right\rangle_{\mathcal{F}}\right| \lesssim \alpha^{-1}\|\Phi\|_{\mathcal{F}}
$$

This yields a bound of the form (7-3). 
We now bound the integrand in $M_{11}$. We have

$$
\begin{aligned}
& \left|\left\langle\tilde{\psi}_{t} \otimes \Phi_{s, t}, e^{-i H_{\varphi_{t}}\left(t-s-s_{1}\right)}\left(\partial_{t} V_{\varphi_{t}}\right) e^{-i H_{\varphi_{t}} s_{1}} \int_{\mathbb{R}^{3}}\left(e^{i k \cdot x} b_{k}^{*} \tilde{\psi}_{s} \otimes \Omega\right) \frac{d k}{|k|}\right\rangle_{\mathcal{L}^{2} \otimes \mathcal{F}}\right| \\
& \leq\left\|\left(H_{\varphi_{t}}+M\right)^{\frac{1}{2}} \tilde{\psi}_{t} \otimes \Phi_{s, t}\right\|\left\|\left(H_{\varphi_{t}}+M\right)^{-\frac{1}{2}}\left(\partial_{t} V_{\varphi_{t}}\right)\left(H_{\varphi_{t}}+M\right)^{\frac{1}{2}}\right\|\left\|\left(H_{\varphi_{t}}+M\right)^{-\frac{1}{2}} \int_{\mathbb{R}^{3}}\left(e^{i k \cdot x} b_{k}^{*} \tilde{\psi}_{s} \otimes \Omega\right) \frac{d k}{|k|}\right\| .
\end{aligned}
$$

By Corollary B.2 and an easy modification of its proof, for $M$ sufficiently large (but independent of $t$ and $\alpha$ ), the operators $\left(H_{\varphi_{t}}+M\right)^{ \pm \frac{1}{2}}(-\Delta+1)^{\mp \frac{1}{2}}$ are both bounded uniformly in $t$. Therefore Lemma 3.1 and the a priori bounds from Lemma 2.1 yield

$$
\begin{aligned}
&\left|\left\langle\tilde{\psi}_{t} \otimes \Phi_{s, t}, e^{-i H_{\varphi_{t}}\left(t-s-s_{1}\right)}\left(\partial_{t} V_{\varphi_{t}}\right) e^{-i H_{\varphi_{t}} s_{1}} \int_{\mathbb{R}^{3}}\left(e^{i k \cdot x} b_{k}^{*} \tilde{\psi}_{s} \otimes \Omega\right) \frac{d k}{|k|}\right\rangle_{\mathcal{L}^{2} \otimes \mathcal{F}}\right| \\
& \\
& \lesssim \alpha^{-1}\left\|\tilde{\psi}_{t}\right\|_{\mathcal{H}^{1}}\|\Phi\|_{\mathcal{F}}\left\|(-\Delta+1)^{-\frac{1}{2}}\left(\partial_{t} V_{\varphi_{t}}\right)(-\Delta+1)^{\frac{1}{2}}\right\|\left\|\psi_{s}\right\|_{\mathcal{H}^{1}} \\
& \lesssim \alpha^{-1}\|\Phi\|_{\mathcal{F}}\left\|(-\Delta+1)^{-\frac{1}{2}}\left(\partial_{t} V_{\varphi_{t}}\right)(-\Delta+1)^{\frac{1}{2}}\right\| .
\end{aligned}
$$

Finally, using the fact that $\left\|\nabla \partial_{t} V_{\varphi_{t}}\right\|_{\infty} \lesssim \alpha^{-2}$ (see (C-8)), we obtain that the operator appearing in this bound has norm $\lesssim \alpha^{-2}$. Thus, we finally obtain

$$
\left|\left\langle\tilde{\psi}_{t} \otimes \Phi_{s, t}, e^{-i H_{\varphi_{t}}\left(t-s-s_{1}\right)}\left(\partial_{t} V_{\varphi_{t}}\right) e^{-i H_{\varphi_{t}} s_{1}} \int_{\mathbb{R}^{3}}\left(e^{i k \cdot x} b_{k}^{*} \tilde{\psi}_{s} \otimes \Omega\right) \frac{d k}{|k|}\right\rangle_{\mathcal{L}^{2} \otimes \mathcal{F}}\right| \lesssim \alpha^{-3},
$$

which, when integrated over $s_{1}$ and $s$, leads to the bound in (7-3).

Bound on $M_{2}$. As for $M_{1}$, we use $P \underset{\tilde{\psi}_{s}}{\perp}=1-\left|\tilde{\psi}_{s}\right\rangle\left\langle\tilde{\psi}_{s}\right|$ to get the decomposition

$$
M_{2}=M_{21}-M_{22}
$$

with

$$
M_{21}(t):=\alpha^{2} \int_{0}^{t}\left\langle\tilde{\psi}_{t} \otimes \Phi_{s, t}, e^{-i H_{\varphi_{t}}(t-s)} \int_{\mathbb{R}^{3}}\left(e^{i k \cdot x}\left(b\left(\partial_{t} \varphi_{t}\right)-b^{*}\left(\partial_{t} \varphi_{t}\right)\right) b_{k}^{*} \tilde{\psi}_{s} \otimes \Omega\right) \frac{d k}{|k|}\right\rangle d s
$$

and, with $\sigma_{\tilde{\psi}_{s}}$ from (2-2),

$$
M_{22}(t):=\alpha^{2} \int_{0}^{t}\left\langle\tilde{\psi}_{t}, e^{-i H_{\varphi_{t}}(t-s)} \tilde{\psi}_{s}\right\rangle_{\mathcal{L}^{2}}\left\langle\Phi_{s, t},\left(b\left(\partial_{t} \varphi_{t}\right)-b^{*}\left(\partial_{t} \varphi_{t}\right)\right) b^{*}\left(\sigma_{\tilde{\psi}_{s}}\right) \Omega\right\rangle_{\mathcal{F}} d s .
$$

Once again the bound on $M_{22}$ is straightforward. Namely, we commute $b^{*}\left(\sigma_{\tilde{\psi}_{s}}\right)$ to the left through $b\left(\partial_{t} \varphi_{t}\right)-b^{*}\left(\partial_{t} \varphi_{t}\right)$ and obtain

$\left\langle\Phi_{s, t},\left(b\left(\partial_{t} \varphi_{t}\right)-b^{*}\left(\partial_{t} \varphi_{t}\right)\right) b^{*}\left(\sigma_{\tilde{\psi}_{s}}\right) \Omega\right\rangle_{\mathcal{F}}=-\left\langle\Phi_{s, t}, b^{*}\left(\sigma_{\tilde{\psi}_{s}}\right) b^{*}\left(\partial_{t} \varphi_{t}\right) \Omega\right\rangle_{\mathcal{F}}+\alpha^{-2}\left(\partial_{t} \varphi_{t}, \sigma_{\tilde{\psi}_{s}}\right)\left\langle\Phi_{s, t}, \Omega\right\rangle_{\mathcal{F}}$.

By similar computations as, for instance, in the bound on $D_{32}$ and by the a priori bounds from Lemma 2.1, we obtain

$$
\left|\left\langle\Phi_{s, t},\left(b\left(\partial_{t} \varphi_{t}\right)-b^{*}\left(\partial_{t} \varphi_{t}\right)\right) b^{*}\left(\sigma_{\widetilde{\psi}_{s}}\right) \Omega\right\rangle_{\mathcal{F}}\right| \lesssim \alpha^{-2}\|\Phi\|_{\mathcal{F}}\left\|\sigma_{\widetilde{\psi}_{s}}\right\|\left\|\partial_{t} \varphi_{t}\right\| \lesssim \alpha^{-4}\|\Phi\|_{\mathcal{F}}
$$


By the conservation of the $\mathcal{L}^{2}$ norm of $\tilde{\psi}_{t}$ we conclude

$$
\left|M_{22}(t)\right| \lesssim \alpha^{-2} t\|\Phi\|_{\mathcal{F}},
$$

which is of the form claimed in (7-3).

We now discuss $M_{21}$. Again we commute $b_{k}^{*}$ to the left through $b\left(\partial_{t} \varphi_{t}\right)-b^{*}\left(\partial_{t} \varphi_{t}\right)$ and obtain

$$
M_{21}=M_{211}+M_{212}
$$

where

$$
M_{211}(t):=-\alpha^{2} \int_{0}^{t}\left\langle\tilde{\psi}_{t} \otimes \Phi_{s, t}, e^{-i H_{\varphi_{t}}(t-s)} \int_{\mathbb{R}^{3}}\left(e^{i k \cdot x} b_{k}^{*} b^{*}\left(\partial_{t} \varphi_{t}\right) \tilde{\psi}_{s} \otimes \Omega\right) \frac{d k}{|k|}\right\rangle_{\mathcal{L}^{2} \otimes \mathcal{F}} d s
$$

and, with $g_{s}$ from (2-6),

$$
M_{212}(t):=\int_{0}^{t}\left\langle\tilde{\psi}_{t}, e^{-i H_{\varphi_{t}}(t-s)} g_{s} \tilde{\psi}_{s}\right\rangle_{\mathcal{L}^{2}}\left\langle\Phi_{s, t}, \Omega\right\rangle_{\mathcal{F}} d s .
$$

Since $\left\|g_{s}\right\|_{\infty} \lesssim \alpha^{-2}$ by Proposition 2.2, we obtain immediately

$$
\left|M_{212}(t)\right| \lesssim \alpha^{-2} t\|\Phi\|_{\mathcal{F}}
$$

To control $M_{211}$ we bound

$$
\begin{aligned}
\mid\left\langle\tilde{\psi}_{t} \otimes \Phi_{s, t}, e^{-i H_{\varphi_{t}}(t-s)}\right. & \left.\int_{\mathbb{R}^{3}}\left(e^{i k \cdot x} b_{k}^{*} b^{*}\left(\partial_{t} \varphi_{t}\right) \tilde{\psi}_{s} \otimes \Omega\right) \frac{d k}{|k|}\right\rangle_{\mathcal{L}^{2} \otimes \mathcal{F}} \mid \\
\leq & \left\|\left(H_{\varphi_{t}}+M\right)^{\frac{1}{2}} \tilde{\psi}_{t} \otimes \Phi_{s, t}\right\|\left\|\left(H_{\varphi_{t}}+M\right)^{-\frac{1}{2}} \int_{\mathbb{R}^{3}}\left(e^{i k \cdot x} b_{k}^{*} b^{*}\left(\partial_{t} \varphi_{t}\right) \tilde{\psi}_{s} \otimes \Omega\right) \frac{d k}{|k|}\right\| .
\end{aligned}
$$

As for $M_{11}$, we use Lemma 2.1 and Corollary B.2 (and a simple extension of its proof) to choose $M$ large enough, but independent of $t$ and $\alpha$, so that $\left(H_{\varphi_{t}}+M\right)^{ \pm \frac{1}{2}}(-\Delta+1)^{\mp \frac{1}{2}}$ are both bounded uniformly in $t$. Therefore Lemma 3.1 and the a priori bounds from Lemma 2.1 yield

$$
\begin{aligned}
\left|\left\langle\tilde{\psi}_{t} \otimes \Phi_{s, t}, e^{-i \boldsymbol{H}_{\varphi_{t}}(t-s)} \int_{\mathbb{R}^{3}}\left(e^{i k \cdot x} b_{k}^{*} b^{*}\left(\partial_{t} \varphi_{t}\right) \tilde{\psi}_{s} \otimes \Omega\right) \frac{d k}{|k|}\right\rangle_{\mathcal{L}^{2} \otimes \mathcal{F}}\right| & \lesssim \alpha^{-2}\left\|\tilde{\psi}_{t}\right\|_{\mathcal{H}^{1}}\|\Phi\|_{\mathcal{F}}\left\|\partial_{t} \varphi_{t}\right\|_{\mathcal{L}^{2}}\left\|\tilde{\psi}_{s}\right\|_{\mathcal{H}^{1}} \\
& \lesssim \alpha^{-4}\|\Phi\|_{\mathcal{F} .}
\end{aligned}
$$

This, when integrated over $s$ and multiplied by $\alpha^{2}$, leads to the bound in (7-3).

Bound on $\mathbf{M}_{3}$. The a priori bounds from Lemma 2.1 yield

$$
|m(s, t)| \lesssim \alpha^{-2}|t-s| .
$$

Moreover, applying Lemma 3.1 as in the bound on $M_{21}$ we find that the absolute value of the inner product in the integral defining $M_{3}$ is bounded by a constant times $\alpha^{-1}\|\Phi\|_{\mathcal{F}}$. This yields the bound in (7-3).

This concludes the proof of (2-29). 


\section{Appendix A: Some properties of the Weyl operators}

In this appendix we collect some standard properties of the Weyl operators $W(f)$ defined in (1-7) in terms of $b(f)$ and $b^{*}(f)$. They are well known, but we provide proofs for the sake of completeness. We recall that the commutation relations for $b_{k}$ and $b_{k}^{*}$ involve a factor $\alpha^{-2}$.

Lemma A.1. The operators $b_{k}, b_{k}^{*}$ and $W(f)$ satisfy the following relations,

$$
b_{k} W(f)=W(f)\left(b_{k}+\alpha^{-2} f(k)\right) \quad \text { and } \quad b_{k}^{*} W(f)=W(f)\left(b_{k}^{*}+\alpha^{-2} \bar{f}(k)\right) .
$$

Proof. For $t>0$ we consider the operators

$$
F_{t}:=W(t f)=e^{t\left(b^{*}(f)-b(f)\right)},
$$

which satisfy

$$
\partial_{t} F_{t}=\left(b^{*}(f)-b(f)\right) F_{t}, \quad F_{0}=\text { Id } .
$$

Multiplying by $b_{k}$ and using the commutation relations, we obtain the following equation for $b_{k} F_{t}$ :

$$
\partial_{t} b_{k} F_{t}=\left(b^{*}(f)-b(f)\right) b_{k} F_{t}+\alpha^{-2} f(k) F_{t}, \quad b_{k} F_{0}=b_{k} .
$$

Therefore, by Duhamel's principle applied to the latter equation,

$$
b_{k} F_{t}=e^{t\left(b^{*}(f)-b(f)\right)} b_{k}+\alpha^{-2} f(k) \int_{0}^{t} e^{(t-s)\left(b^{*}(f)-b(f)\right)} F_{s} d s .
$$

Recalling the definition of $F_{t}$ in (A-2), we can rewrite this as

$$
b_{k} F_{t}=F_{t} b_{k}+t \alpha^{-2} f(k) F_{t} .
$$

At $t=1$ we obtain the first identity in the lemma. The second one is proved similarly.

By applying Lemma A.1 twice, we obtain:

Corollary A.2.

$$
\begin{aligned}
& {\left[b_{k}^{*}, W^{*}(f) W(g)\right]=-\alpha^{-2}(\bar{f}(k)-\bar{g}(k)) W^{*}(f) W(g),} \\
& {\left[b_{k}, W^{*}(f) W(g)\right]=-\alpha^{-2}(f(k)-g(k)) W^{*}(f) W(g) .}
\end{aligned}
$$

Next, we'll consider the case where $f$ depends (differentiably) on a parameter.

\section{Lemma A.3.}

$$
\begin{aligned}
& \partial_{t} W\left(f_{t}\right)=\frac{1}{2} \alpha^{-2}\left(\left(f_{t}, \partial_{t} f_{t}\right)-\left(\partial_{t} f_{t}, f_{t}\right)\right) W\left(f_{t}\right)+W\left(f_{t}\right)\left(b^{*}\left(\partial_{t} f_{t}\right)-b\left(\partial_{t} f_{t}\right)\right), \\
& \partial_{t} W\left(f_{t}\right)=-\frac{1}{2} \alpha^{-2}\left(\left(f_{t}, \partial_{t} f_{t}\right)-\left(\partial_{t} f_{t}, f_{t}\right)\right) W\left(f_{t}\right)+\left(b^{*}\left(\partial_{t} f_{t}\right)-b\left(\partial_{t} f_{t}\right)\right) W\left(f_{t}\right) .
\end{aligned}
$$

Proof. For $s>0$ we consider the operators

$$
F(s, t):=W\left(s f_{t}\right),
$$

which satisfy

$$
\partial_{s} F(s, t)=\left(b^{*}\left(f_{t}\right)-b\left(f_{t}\right)\right) F(s, t), \quad F(0, t)=\mathrm{Id} .
$$


We differentiate this equation with respect to $t$ and obtain

$$
\begin{aligned}
\partial_{s} \partial_{t} F(s, t) & =\left(b^{*}\left(f_{t}\right)-b\left(f_{t}\right)\right) \partial_{t} F(s, t)+\left(b^{*}\left(\partial_{t} f_{t}\right)-b\left(\partial_{t} f_{t}\right)\right) F(s, t), \\
\partial_{t} F(0, t) & =0 .
\end{aligned}
$$

Therefore, by Duhamel's principle,

$$
\begin{aligned}
\partial_{t} F(s, t) & =\int_{0}^{s} e^{\left(b^{*}\left(f_{t}\right)-b\left(f_{t}\right)\right)\left(s-s_{1}\right)}\left(b^{*}\left(\partial_{t} f_{t}\right)-b\left(\partial_{t} f_{t}\right)\right) F\left(s_{1}, t\right) d s_{1} \\
& =\int_{0}^{s} W\left(\left(s-s_{1}\right) f_{t}\right)\left(b^{*}\left(\partial_{t} f_{t}\right)-b\left(\partial_{t} f_{t}\right)\right) W\left(s_{1} f_{t}\right) d s_{1} .
\end{aligned}
$$

In order to simplify the integrand we now use Lemma A.1 and obtain

$\left(b^{*}\left(\partial_{t} f_{t}\right)-b\left(\partial_{t} f_{t}\right)\right) W\left(s_{1} f_{t}\right)=\alpha^{-2} W\left(s_{1} f_{t}\right) s_{1}\left(\left(f_{t}, \partial_{t} f_{t}\right)-\left(\partial_{t} f_{t}, f_{t}\right)\right)+W\left(s_{1} f_{t}\right)\left(b^{*}\left(\partial_{t} f_{t}\right)-b\left(\partial_{t} f_{t}\right)\right)$.

If we insert this into the above formula for $\partial_{t} F(s, t)$, we obtain

$$
\partial_{t} F(s, t)=\alpha^{-2} \frac{1}{2} s^{2} W\left(s f_{t}\right)\left(\left(f_{t}, \partial_{t} f_{t}\right)-\left(\partial_{t} f_{t}, f_{t}\right)\right)+s W\left(s f_{t}\right)\left(b^{*}\left(\partial_{t} f_{t}\right)-b\left(\partial_{t} f_{t}\right)\right) .
$$

At $s=1$, we obtain the first identity in the lemma. The second one is proved similarly.

Lemma A.4. For any $f, g \in \mathcal{L}^{2}$,

$$
\left\langle\Omega, W^{*}(g) W(f) \Omega\right\rangle=e^{i \alpha^{-2} \operatorname{Im}(g, f)-\alpha^{-2}\|f-g\|^{2} / 2} .
$$

Proof. Let $f_{t}:=t f+(1-t) g$ and $F(t):=\left\langle\Omega, W^{*}(g) W\left(f_{t}\right) \Omega\right\rangle$. By Lemma A.3, using that $\operatorname{Im}\left(f_{t}, \partial_{t} f_{t}\right)=\operatorname{Im}\left(f_{t}, f-g\right)=\operatorname{Im}(g, f)$,

$$
\partial_{t} F(t)=\left\langle\Omega, W^{*}(g) W\left(f_{t}\right)\left(b^{*}(f-g)+i \alpha^{-2} \operatorname{Im}(g, f)\right) \Omega\right\rangle .
$$

Next, by Corollary A.2, since $\left(g-f_{t}, f-g\right)=-t\|f-g\|^{2}$,

$$
W^{*}(g) W\left(f_{t}\right) b^{*}(f-g)=b^{*}(f-g) W^{*}(g) W\left(f_{t}\right)+\alpha^{-2}\left(g-f_{t}, f-g\right) W^{*}(g) W\left(f_{t}\right),
$$

so

$$
\partial_{t} F(t)=\left(-\alpha^{-2} t\|f-g\|^{2}+i \alpha^{-2} \operatorname{Im}(g, f)\right) F(t) .
$$

Since $F(0)=1$, we conclude that

$$
F(t)=e^{-\alpha^{-2} t^{2}\|f-g\|^{2} / 2+i \alpha^{-2} t \operatorname{Im}(g, f),}
$$

which, at $t=1$, gives the assertion.

\section{Appendix B: The effective Schrödinger operator}

In this appendix we investigate the operator and form domains of the effective Schrödinger operator $H_{\varphi}$ from (2-12) with potential $V_{\varphi}$ from (2-1). 
Lemma B.1. For every $A>0$ and $\varepsilon>0$ there is an $M>0$ such that if $\|\varphi\| \leq A$, then for all $\psi \in \mathcal{H}^{1}\left(\mathbb{R}^{3}\right)$,

$$
\left\|\left|V_{\varphi}\right|^{\frac{1}{2}} \psi\right\| \leq \varepsilon\left\|(-\Delta+M)^{\frac{1}{2}} \psi\right\|
$$

and for all $\psi \in \mathcal{H}^{2}\left(\mathbb{R}^{3}\right)$,

$$
\left\|V_{\varphi} \psi\right\| \leq \varepsilon\|(-\Delta+M) \psi\| .
$$

Proof. As in [Frank and Schlein 2014, Section 2.1], the Hardy-Littlewood-Sobolev inequality implies

$$
\left\|V_{\varphi}\right\|_{6} \lesssim\|\varphi\|_{2}
$$

This implies, by the Hölder and Sobolev inequalities,

$$
\int_{\mathbb{R}^{3}}\left|V_{\varphi}\right||\psi|^{2} d x \leq\left\|V_{\varphi}\right\|_{6}\|\psi\|_{\frac{12}{5}}^{2} \lesssim\|\varphi\|_{2}\|\nabla \psi\|_{2}^{\frac{1}{2}}\|\psi\|_{2}^{\frac{3}{2}}
$$

and

$$
\int_{\mathbb{R}^{3}}\left|V_{\varphi}\right|^{2}|\psi|^{2} d x \leq\left\|V_{\varphi}\right\|_{6}^{2}\|\psi\|_{3}^{2} \lesssim\|\varphi\|_{2}^{2}\|\Delta \psi\|_{2}^{\frac{1}{2}}\|\psi\|_{2}^{\frac{3}{2}} .
$$

These bounds easily imply the assertions of the lemma.

Corollary B.2. For every $A>0$ there are $M>0$ and $C>0$ such that if $\|\varphi\|_{2} \leq A$ then for all $f \in \mathcal{L}^{2}\left(\mathbb{R}^{3}\right)$

$$
\left\|\left(H_{\varphi}+M\right)^{-\frac{1}{2}} f\right\|_{2} \leq C\left\|(-\Delta+1)^{-\frac{1}{2}} f\right\|_{2}
$$

and

$$
\left\|\left(H_{\varphi}+M\right)^{-1} f\right\|_{2} \leq C\left\|(-\Delta+1)^{-1} f\right\|_{2} .
$$

Proof. To prove the first assertion, we write

$$
\left(H_{\varphi}+M\right)^{-1}=(-\Delta+M)^{-\frac{1}{2}}\left(1+(-\Delta+M)^{-\frac{1}{2}} V_{\varphi}(-\Delta+M)^{-\frac{1}{2}}\right)^{-1}(-\Delta+M)^{-\frac{1}{2}}
$$

and note that according to Lemma B.1 we can choose $M$ such that $\|\varphi\| \leq A$ implies

$$
\left\|(-\Delta+M)^{-\frac{1}{2}} V_{\varphi}(-\Delta+M)^{-\frac{1}{2}}\right\| \leq \varepsilon^{2} .
$$

Similarly, for the second assertion we write

$$
\left(H_{\varphi}+M\right)^{-1}=\left(1+(-\Delta+M)^{-1} V_{\varphi}\right)^{-1}(-\Delta+M)^{-1}
$$

and choose $M$ such that $\|\varphi\| \leq A$ implies $\left\|(-\Delta+M)^{-1} V_{\varphi}\right\| \leq \varepsilon$.

\section{Appendix C: Well-posedness of the Landau-Pekar equations}

In this appendix we prove Lemma 2.1 and Proposition 2.2. Recall that the weighted spaces $\mathcal{L}_{(m)}^{2}=$ $\mathcal{L}^{2}\left(\mathbb{R}^{3} ;\left(1+k^{2}\right)^{m} d k\right)$ were introduced in (1-11). We begin with some bounds on the coupling terms $V_{\varphi}$ and $\sigma_{\psi}$ introduced in (2-1) and (2-2). 
Lemma C.1. We have

$$
\begin{gathered}
\left\|\partial^{\beta} V_{\varphi}\right\|_{\infty} \lesssim\|\varphi\|_{\mathcal{L}_{|\beta|+1}^{2}} \quad \text { for all } \beta \in \mathbb{N}_{0}^{3}, \\
\left\|\sigma_{\psi}\right\|_{\mathcal{L}_{(1)}^{2}} \lesssim\|\psi\|_{\mathcal{H}^{1}}^{2}, \quad\left\|\sigma_{\psi}\right\|_{\mathcal{L}_{(3)}^{2}}^{2} \lesssim\|\psi\|_{\mathcal{H}^{2}}^{2}
\end{gathered}
$$

Proof. By the Schwarz inequality,

$$
\left|\partial^{\beta} V_{\varphi}(x)\right| \leq 2 \int_{\mathbb{R}^{3}}|k|^{|\beta|-1}|\varphi(k)| d k \leq 2\|\varphi\|_{\mathcal{L}_{|\beta|+1}^{2}}\left(\int_{\mathbb{R}^{3}} \frac{|k|^{2(|\beta|-1)} d k}{\left(1+k^{2}\right)^{2(|\beta|+1)}}\right)^{\frac{1}{2}}
$$

and the last integral is finite.

We have

$$
\left\|\sigma_{\psi}\right\|_{2}^{2}=\left\|\frac{1}{|k|} \int_{\mathbb{R}^{3}}|\psi(x)|^{2} e^{i k \cdot x} d x\right\|_{2}^{2}=2 \pi^{2} \iint_{\mathbb{R}^{3} \times \mathbb{R}^{3}} \frac{|\psi(x)|^{2}|\psi(y)|^{2}}{|x-y|} d x d y .
$$

By the Hardy-Littlewood-Sobolev inequality, we know this is bounded by a constant times $\left\||\psi|^{2}\right\|_{\frac{6}{5}}^{2}=$ $\|\psi\|_{\frac{12}{5}}^{4}$, which, by the Sobolev embedding theorem, is bounded by a constant times $\|\psi\|_{\mathcal{H}^{1}}^{4}$. Moreover, by Plancherel,

$$
\left\|\sigma_{\psi}\right\|_{\mathcal{L}^{2}\left(|k|^{2 m}\right)}^{2}=\left.\left.\int_{\mathbb{R}^{3}}|k|^{2(m-1)}\left|\int_{\mathbb{R}^{3}}\right| \psi\right|^{2} e^{i k \cdot x} d x\right|^{2} d k=(2 \pi)^{3}\left(|\psi|^{2},(-\Delta)^{m-1}|\psi|^{2}\right) .
$$

In particular, for $m=1$ we get $\|\psi\|_{4}^{4}$, which by Sobolev is controlled by $\|\psi\|_{\mathcal{H}^{1}}^{2}$. For $m=3$, the claimed bound follows easily using $\|\psi\|_{\infty} \lesssim\|\psi\|_{\mathcal{H}^{2}}$ and again Sobolev.

Proof of Lemma 2.1. Local well-posedness in $\mathcal{H}^{1} \times \mathcal{L}^{2}$ follows by a standard fixed-point argument and one sees that $\left\|\psi_{t}\right\|_{2}$ and $\mathcal{E}\left(\psi_{t}, \varphi_{t}\right)$ are conserved. One can use (B-1) and the Sobolev inequality to show that [Frank and Schlein 2014, Section 2.1],

$$
\mathcal{E}(\psi, \varphi) \geq\|\nabla \psi\|_{2}^{2}+\|\varphi\|_{2}^{2}-C\|\varphi\|_{2}\|\nabla \psi\|_{2}^{\frac{1}{2}}\|\psi\|_{2}^{\frac{3}{2}}
$$

for some universal constant $C>0$. This, together with conservation of $\mathcal{E}\left(\psi_{t}, \varphi_{t}\right)$, yields global wellposedness as well as the uniform bounds (2-3).

According to (C-2) and the first bound in (2-3), we have $\left\|\sigma_{\psi_{t}}\right\| \lesssim\left\|\psi_{t}\right\|_{\mathcal{H}^{1}}^{2} \lesssim 1$, which is the third bound in (2-4).

By equation (1-9) for $\varphi_{t}$ we have

$$
\left\|\alpha^{2} \partial_{t} \varphi_{t}\right\|_{2} \leq\left\|\varphi_{t}\right\|_{2}+\left\|\sigma_{\psi_{t}}\right\|_{2}
$$

and therefore, by the second bound in (2-3) and the third bound in (2-4), we obtain the first bound in (2-4).

Finally, $\varphi_{t}-\varphi_{s}=\int_{s}^{t} \partial_{s_{1}} \varphi_{s_{1}} d s_{1}$, so for $t>s$, by the first bound in (2-4),

$$
\left\|\varphi_{t}-\varphi_{s}\right\|_{2} \leq \int_{s}^{t}\left\|\partial_{s_{1}} \varphi_{s_{1}}\right\|_{2} d s_{1} \lesssim \alpha^{-2}|t-s|
$$

This proves the second bound in (2-4) and completes the proof of the lemma.

Before dealing with $\mathcal{H}^{4} \times \mathcal{L}_{(3)}^{2}$-regularity in Proposition 2.2 , we need to establish $\mathcal{H}^{2} \times \mathcal{L}_{(1)}^{2}$-regularity. 
Lemma C.2. If $\left(\psi_{0}, \varphi_{0}\right) \in \mathcal{H}^{2}\left(\mathbb{R}^{3}\right) \times \mathcal{L}_{(1)}^{2}\left(\mathbb{R}^{3}\right)$, then $\left(\psi_{t}, \varphi_{t}\right) \in \mathcal{H}^{2}\left(\mathbb{R}^{3}\right) \times \mathcal{L}_{(1)}^{2}\left(\mathbb{R}^{3}\right)$ for all $t \in \mathbb{R}$ and

$$
\left\|\psi_{t}\right\|_{\mathcal{H}^{2}} \lesssim 1+\alpha^{-2}|t|, \quad\left\|\varphi_{t}\right\|_{\mathcal{L}_{(1)}^{2}\left(\mathbb{R}^{3}\right)} \lesssim 1+\alpha^{-2}|t|
$$

with implicit constants depending only on the initial data. Moreover,

$$
\left\|\partial_{t} \psi_{t}\right\|_{\mathcal{L}^{2}} \lesssim 1+\alpha^{-2}|t|, \quad\left\|\partial_{t} \sigma_{\psi_{t}}\right\|_{\mathcal{L}^{2}} \lesssim 1+\alpha^{-2}|t| .
$$

If , in addition, $\varphi_{0} \in \mathcal{L}_{(m)}^{2}\left(\mathbb{R}^{3}\right), m=2,3$, then $\varphi_{t} \in \mathcal{L}_{(m)}^{2}\left(\mathbb{R}^{3}\right)$ for all $t \in \mathbb{R}$ and

$$
\left\|\varphi_{t}\right\|_{\mathcal{L}_{(m)}^{2}\left(\mathbb{R}^{3}\right)} \lesssim 1+\alpha^{-6}|t|^{3} .
$$

Proof. By a standard fixed-point argument one can show local existence of solutions in $\mathcal{H}^{2} \times \mathcal{L}_{(1)}^{2}$. In the following we will construct a functional, which is equivalent to the $\mathcal{H}^{2}$ norm of $\psi$ and which grows in a controlled way as time increases. This will prove, in particular, that $\psi_{t}$ belongs to $\mathcal{H}^{2}$ for all times.

We claim that for every $A>0$ there is a constant $M>0$ such that

$$
\mathcal{E}^{(2)}(\psi, \varphi):=\left\|\left(-\Delta+V_{\varphi}+M\right) \psi\right\|_{2}^{2}
$$

satisfies

$$
\frac{1}{2}\|\psi\|_{\mathcal{H}^{2}} \leq\left(\mathcal{E}^{(2)}(\psi, \varphi)\right)^{\frac{1}{2}} \leq \frac{3}{2}\|\psi\|_{\mathcal{H}^{2}}
$$

for all $\psi \in \mathcal{H}^{2}$ and all $\varphi$ satisfying $\|\varphi\|_{2} \leq A$. In fact, much as in the proof of Corollary B.2, we have

$$
\left|\left\|\left(-\Delta+V_{\varphi}+M\right) \psi\right\|_{2}-\|(-\Delta+M) \psi\|_{2}\right| \leq\left\|V_{\varphi}(-\Delta+M)^{-1}\right\|\|(-\Delta+M) \psi\|_{2}
$$

and according to Lemma B.1 we can choose $M$ such that the first factor on the right side is less than $\varepsilon$ for $\|\varphi\|_{2} \leq A$.

According to Lemma 2.1 there is an $A>0$ (depending only on $\left\|\psi_{0}\right\|_{\mathcal{H}^{1}}$ and $\left\|\varphi_{0}\right\|_{\mathcal{L}^{2}}$ ) such that $\left\|\varphi_{t}\right\|_{\mathcal{L}^{2}} \leq A$ for all $t$. We choose $M$ corresponding to this value of $A$ and compute, using the equation for $\psi_{t}$,

$$
\begin{aligned}
\partial_{t} \mathcal{E}^{(2)} & \left(\psi_{t}, \varphi_{t}\right) \\
& =2 \operatorname{Re}\left(\left(-\Delta+V_{\varphi_{t}}+M\right) \psi_{t},\left(-\Delta+V_{\varphi_{t}}+M\right) \partial_{t} \psi_{t}\right)+2 \operatorname{Re}\left(\left(-\Delta+V_{\varphi_{t}}+M\right) \psi_{t},\left(\partial_{t} V_{\varphi_{t}}\right) \psi_{t}\right) \\
& =2 \operatorname{Re}\left(\left(-\Delta+V_{\varphi_{t}}+M\right) \psi_{t},\left(\partial_{t} V_{\varphi_{t}}\right) \psi_{t}\right) .
\end{aligned}
$$

By the Schwarz and the Hölder inequalities,

$$
\partial_{t} \mathcal{E}^{(2)}\left(\psi_{t}, \varphi_{t}\right) \leq 2\left(\mathcal{E}^{(2)}\left(\psi_{t}, \varphi_{t}\right)\right)^{\frac{1}{2}}\left\|\partial_{t} V_{\varphi_{t}}\right\|_{6}\left\|\psi_{t}\right\|_{3} .
$$

By (B-1) and Lemma 2.1, $\left\|\partial_{t} V_{\varphi_{t}}\right\|_{6} \lesssim\left\|\partial_{t} \varphi_{t}\right\|_{2} \lesssim \alpha^{-2}$, and by the Sobolev inequality and Lemma 2.1, $\left\|\psi_{t}\right\|_{3} \lesssim\left\|\psi_{t}\right\|_{\mathcal{H}^{1}} \lesssim 1$. Thus,

$$
\partial_{t} \mathcal{E}^{(2)}\left(\psi_{t}, \varphi_{t}\right) \lesssim \alpha^{-2}\left(\mathcal{E}^{(2)}\left(\psi_{t}, \varphi_{t}\right)\right)^{\frac{1}{2}},
$$

which implies $\left(\mathcal{E}^{(2)}\left(\psi_{t}, \varphi_{t}\right)\right)^{\frac{1}{2}} \lesssim 1+\alpha^{-2}|t|$. According to (C-5), this implies the claimed bound on $\left\|\psi_{t}\right\|_{\mathcal{H}^{2}}$.

The remaining bounds are proved in a straightforward way. We have

$$
\left\|\partial_{t} \psi_{t}\right\|_{2} \leq\left\|-\Delta \psi_{t}\right\|_{2}+\left\|V_{\varphi_{t}} \psi_{t}\right\|_{2} \leq\left\|\psi_{t}\right\|_{\mathcal{H}^{2}}+\left\|V_{\varphi_{t}}\right\|_{6}\left\|\psi_{t}\right\|_{3} .
$$


By the bound on $\left\|\psi_{t}\right\|_{\mathcal{H}^{2}}$ together with (B-1) and the bounds from Lemma 2.1, we obtain the first bound in (C-4). Moreover,

$$
\partial_{t} \sigma_{\psi_{t}}=2|k|^{-1} \int_{\mathbb{R}^{3}} \operatorname{Re}\left(\overline{\psi_{t}} \partial_{t} \psi_{t}\right) e^{i k \cdot x} d x
$$

and so, by the Hardy-Littlewood-Sobolev inequality as in (B-1),

$$
\left\|\partial_{t} \sigma_{\psi_{t}}\right\|_{2} \lesssim\left\|\psi_{t} \partial_{t} \psi_{t}\right\|_{\frac{6}{5}} \leq\left\|\psi_{t}\right\|_{3}\left\|\partial_{t} \psi_{t}\right\|_{2}
$$

By the first bound in (C-4) and Lemma 2.1, we obtain the second bound in (C-4).

In order to deduce the bounds on $\varphi_{t}$, we use Duhamel's formula:

$$
\varphi_{t}(k)=e^{-i t / \alpha^{2}} \varphi_{0}(k)-i \alpha^{-2} \int_{0}^{t} e^{-i(t-s) / \alpha^{2}} \sigma_{\psi_{s}}(k) d s .
$$

If $\varphi_{0} \in \mathcal{L}_{(m)}^{2}, m=1,2,3$, we deduce that $\varphi_{t} \in \mathcal{L}_{(m)}^{2}$ provided we can bound $\left\|\sigma_{\psi_{s}}\right\|_{\mathcal{L}_{(m)}^{2}}$. This quantity can by controlled by Sobolev norms of $\psi_{s}$ according to (C-2).

Proof of Proposition 2.2. The basic strategy is the same as in the proof of Lemma C.2, except that verifying the properties of the functional is more complicated in this case. Again we do not give the details of the local existence via a fixed-point argument.

We claim that for every $A>0$ there is a constant $M>0$ such that

$$
\mathcal{E}^{(4)}(\psi, \varphi):=\left\|\left(-\Delta+V_{\varphi}+M\right)^{2} \psi\right\|_{2}^{2}
$$

satisfies

$$
\frac{1}{2}\|\psi\|_{\mathcal{H}^{4}} \leq\left(\mathcal{E}^{(4)}(\psi, \varphi)\right)^{\frac{1}{2}} \leq \frac{3}{2}\|\psi\|_{\mathcal{H}^{4}}
$$

for all $\psi \in \mathcal{H}^{4}$ and all $\varphi$ satisfying $\|\varphi\|_{\mathcal{L}_{(3)}^{2}} \leq A$. To show this, we first observe that, as in the proof of Lemma C.2,

$$
\begin{aligned}
\mid\left\|\left(-\Delta+V_{\varphi}+M\right)^{2} \psi\right\|_{2}-\|(-\Delta+M)(-\Delta+ & \left.V_{\varphi}+M\right) \psi \|_{2} \mid \\
& \leq\left\|V_{\varphi}(-\Delta+M)^{-1}\right\|\left\|(-\Delta+M)\left(-\Delta+V_{\varphi}+M\right) \psi\right\|_{2}
\end{aligned}
$$

and that $\left\|V_{\varphi}(-\Delta+M)^{-1}\right\|$ can be made arbitrarily small for $\|\varphi\|_{\mathcal{L}^{2}}$ bounded by choosing $M$ large. Thus, it suffices to show that $\left\|(-\Delta+M)\left(-\Delta+V_{\varphi}+M\right) \psi\right\|_{2}$ is equivalent to $\left\|(-\Delta+M)^{2} \psi\right\|_{2}$. We compute

$$
\begin{aligned}
\mid\left\|(-\Delta+M)\left(-\Delta+V_{\varphi}+M\right) \psi\right\|_{2}-\|(-\Delta+ & \left.V_{\varphi}+M\right)(-\Delta+M) \psi \|_{2} \mid \\
& \leq\left\|\left(2 \nabla V_{\varphi} \cdot \nabla+\Delta V_{\varphi}\right)(-\Delta+M)^{-1}\right\|\|(-\Delta+M) \psi\|_{2}
\end{aligned}
$$

According to (C-1), the first factor on the right side can be made arbitrarily small for $\|\varphi\|_{\mathcal{L}_{(3)}^{2}}$ bounded by choosing $M$ large. We conclude by applying the argument in Lemma C.2 again to compare $\left\|\left(-\Delta+V_{\varphi}+M\right)(-\Delta+M) \psi\right\|_{2}$ to $\left\|(-\Delta+M)^{2} \psi\right\|_{2}$. This proves the claim.

According to Lemma C.2, for every $\tau>0$ there is an $A>0$ (depending only on $\left\|\psi_{0}\right\|_{\mathcal{H}^{2}},\left\|\varphi_{0}\right\|_{\mathcal{L}_{(3)}^{2}}$ and $\tau$ ) such that $\left\|\varphi_{t}\right\|_{\mathcal{L}_{(3)}^{2}} \leq A$ for all $|t| \leq \tau \alpha^{2}$. We choose $M$ corresponding to this value of $A$ and 
compute, using the equation for $\psi_{t}$,

$$
\begin{aligned}
\partial_{t} \mathcal{E}^{(4)}\left(\psi_{t}, \varphi_{t}\right)=2 \operatorname{Re}\left(\left(-\Delta+V_{\varphi_{t}}+M\right)^{2} \psi_{t},\left(-\Delta+V_{\varphi_{t}}+M\right)^{2} \partial_{t} \psi_{t}\right) \\
+2 \operatorname{Re}\left(\left(-\Delta+V_{\varphi_{t}}+M\right)^{2} \psi_{t},\left(\partial_{t} V_{\varphi_{t}}\right)\left(-\Delta+V_{\varphi_{t}}+M\right) \psi_{t}\right) \\
+2 \operatorname{Re}\left(\left(-\Delta+V_{\varphi_{t}}+M\right)^{2} \psi_{t},\left(-\Delta+V_{\varphi_{t}}+M\right)\left(\partial_{t} V_{\varphi_{t}}\right) \psi_{t}\right) \\
=4 \operatorname{Re}\left(\left(-\Delta+V_{\varphi_{t}}+M\right)^{2} \psi_{t},\left(\partial_{t} V_{\varphi_{t}}\right)\left(-\Delta+V_{\varphi_{t}}+M\right) \psi_{t}\right) \\
-2 \operatorname{Re}\left(\left(-\Delta+V_{\varphi_{t}}+M\right)^{2} \psi_{t},\left(2 \nabla \partial_{t} V_{\varphi_{t}} \cdot \nabla+\Delta \partial_{t} V_{\varphi_{t}}\right) \psi_{t}\right) .
\end{aligned}
$$

Therefore, by the Schwarz inequality,

$$
\begin{aligned}
& \partial_{t} \mathcal{E}^{(4)}\left(\psi_{t}, \varphi_{t}\right) \\
& \quad \leq 2\left(\mathcal{E}^{(4)}\left(\psi_{t}, \varphi_{t}\right)\right)^{\frac{1}{2}}\left(2\left\|\partial_{t} V_{\varphi_{t}}\right\|_{\infty}\left\|\left(-\Delta+V_{\varphi_{t}}+M\right) \psi_{t}\right\|_{2}+2\left\|\nabla \partial_{t} V_{\varphi_{t}}\right\|_{\infty}\left\|\nabla \psi_{t}\right\|_{2}+\left\|\Delta \partial_{t} V_{\varphi_{t}}\right\|_{\infty}\left\|\psi_{t}\right\|_{2}\right) .
\end{aligned}
$$

According to Lemma C.2 and (C-5), all terms involving $\psi_{t}$ here are bounded by a constant for $|t| \leq \tau \alpha^{2}$. Assume that we can prove that all terms involving $\varphi_{t}$ here are bounded by a constant times $\alpha^{-2}$ for $|t| \leq \tau \alpha^{2}$. Then we will have shown that

$$
\partial_{t} \mathcal{E}^{(4)}\left(\psi_{t}, \varphi_{t}\right) \lesssim \alpha^{-2}\left(\mathcal{E}^{(4)}\left(\psi_{t}, \varphi_{t}\right)\right)^{\frac{1}{2}}
$$

for $|t| \leq \tau \alpha^{2}$, which implies that $\left(\mathcal{E}^{(4)}\left(\psi_{t}, \varphi_{t}\right)\right)^{\frac{1}{2}} \lesssim 1+\alpha^{-2}|t| \lesssim 1$ for $|t| \leq \tau \alpha^{2}$. According to (C-7), this proves that $\left\|\psi_{t}\right\|_{\mathcal{H}^{4}} \lesssim 1$ for $|t| \leq \tau \alpha^{2}$.

Thus, it remains to prove that for all multi-indices $\beta$ with $|\beta| \leq 2$,

$$
\left\|\partial_{x}^{\beta} \partial_{t} V_{\varphi_{t}}\right\|_{\infty} \lesssim \alpha^{-2} \text { for }|t| \leq \tau \alpha^{-2}
$$

If we insert the equation of $\varphi_{t}$ into the definition of $V_{\varphi_{t}}$, we find

$$
\partial_{t} V_{\varphi_{t}}(x)=-i \alpha^{-2} \int_{\mathbb{R}^{3}}\left(e^{-i k \cdot x} \varphi_{t}(k)-e^{i k \cdot x} \overline{\varphi_{t}(k)}\right) \frac{d k}{|k|} .
$$

(Note that the contribution from $\sigma_{\psi t}$ cancels.) Using this formula, we obtain

$$
\left\|\partial_{x}^{\beta} \partial_{t} V_{\varphi_{t}}\right\|_{\infty} \lesssim \alpha^{-2}\left\|\varphi_{t}\right\|_{\mathcal{L}_{|\beta|+1}^{2}}
$$

in the same way as we obtained (C-1). This implies (C-8) in view of the bounds on $\varphi_{t}$ from Lemma C.2.

It is straightforward to deduce the remaining bounds claimed in the proposition. The bound on $\left\|\varphi_{t}\right\|_{\mathcal{L}_{(3)}^{2}}$ follows from Lemma C.2. Because of the equation for $\psi_{t}$, we have

$$
\left\|\partial_{t} \psi_{t}\right\|_{\mathcal{H}^{2}} \leq\left\|-\Delta \psi_{t}\right\|_{\mathcal{H}^{2}}+\left\|V_{\varphi_{t}} \psi_{t}\right\|_{\mathcal{H}^{2}} \lesssim\left\|\psi_{t}\right\|_{\mathcal{H}^{4}}+\sum_{|\beta| \leq 2}\left\|\partial^{\beta} V_{\varphi_{t}}\right\|_{\infty}\left\|\psi_{t}\right\|_{\mathcal{H}^{2}}
$$

Using the fact that $\left\|\psi_{t}\right\|_{\mathcal{H}^{4}} \lesssim 1$ and $\left\|\varphi_{t}\right\|_{\mathcal{L}_{(3)}^{2}} \lesssim 1$, which by (C-1) controls $\left\|\partial^{\beta} V_{\varphi_{t}}\right\|_{\infty}$ for $|\beta| \leq 2$, we conclude that $\left\|\partial_{t} \psi_{t}\right\|_{\mathcal{H}^{2}} \lesssim 1$. The second bound in (2-8) follows from Lemma C.2.

Finally, we need to prove the bounds on $g_{s}$ and $g_{s, t}$. By the Schwarz inequality as in the proof of (C-1) together with the equation for $\varphi_{s}$ we find

$$
\left\|g_{s}\right\|_{\infty} \lesssim\left\|\partial_{s} \varphi_{s}\right\|_{\mathcal{L}_{(1)}^{2}} \leq \alpha^{-2}\left(\left\|\varphi_{s}\right\|_{\mathcal{L}_{(1)}^{2}}+\left\|\sigma_{\psi_{s}}\right\|_{\mathcal{L}_{(1)}^{2}}\right)
$$


According to (C-2) and Lemma 2.1 we have $\left\|\sigma_{\psi_{s}}\right\|_{\mathcal{L}_{(1)}^{2}} \lesssim\left\|\psi_{s}\right\|_{H^{1}}^{2} \lesssim 1$. Moreover, if $|t|,|s| \leq \tau \alpha^{2}$, then Lemma C.2 implies $\left\|\varphi_{s}\right\|_{\mathcal{L}_{(1)}^{2}} \lesssim 1$. Thus,

$$
\left\|g_{s}\right\|_{\infty} \lesssim \alpha^{-2}
$$

as claimed. Moreover, $g_{s, t}=\int_{s}^{t} g_{s_{1}} d s_{1}$, so for $t>s$

This proves (2-9).

$$
\left\|g_{s, t}\right\|_{\infty} \leq \int_{s}^{t}\left\|g_{s_{1}}\right\|_{\infty} d s_{1} \lesssim \alpha^{-2}(t-s) .
$$

\section{Appendix D: Reduced density matrices}

Here we show how the approximation of $e^{-i \widetilde{H}_{\alpha}^{F} t} \psi_{0} \otimes W\left(\alpha^{2} \varphi_{0}\right) \Omega$ in Theorem 1.3 yields approximations to its reduced density matrices in Theorem 1.2. The argument relies on the following abstract lemma.

Lemma D.1. Let $\mathcal{H}_{1}$ and $\mathcal{H}_{2}$ be Hilbert spaces; let $\Psi, \Phi \in \mathcal{H}_{1} \otimes \mathcal{H}_{2}$ and $f \in \mathcal{H}_{1}$ and $g \in \mathcal{H}_{2}$ such that

$$
\begin{gathered}
\Psi=f \otimes g+\Phi \\
\|f\|_{\mathcal{H}_{1}} \leq C, \quad\|g\|_{\mathcal{H}_{2}} \leq C, \quad\|\Phi\|_{\mathcal{H}_{1} \otimes \mathcal{H}_{2}} \leq C \varepsilon \\
\left\|\langle g, \Phi\rangle_{\mathcal{H}_{2}}\right\|_{\mathcal{H}_{1}} \leq C \varepsilon^{2}, \quad\left\|\langle f, \Phi\rangle_{\mathcal{H}_{1}}\right\|_{\mathcal{H}_{2}} \leq C \varepsilon^{2}
\end{gathered}
$$

for some $C>0$ and $\varepsilon>0$. Define

$$
\gamma_{1}=\operatorname{Tr}_{\mathcal{H}_{2}}|\Psi\rangle\left\langle\Psi\left|, \quad \gamma_{2}=\operatorname{Tr}_{\mathcal{H}_{1}}\right| \Psi\right\rangle\langle\Psi|
$$

Then

$$
\left.\operatorname{Tr}_{\mathcal{H}_{1}}\left|\gamma_{1}-\|g\|_{\mathcal{H}_{2}}^{2}\right| f\right\rangle\left\langlef \| \leq 3 C ^ { 2 } \varepsilon ^ { 2 } , \quad \operatorname { T r } _ { \mathcal { H } _ { 2 } } | \gamma _ { 2 } - \| f \| _ { \mathcal { H } _ { 1 } } ^ { 2 } | g \rangle \left\langle g \| \leq 3 C^{2} \varepsilon^{2}\right.\right.
$$

Before proving this lemma, let us use it to derive Theorem 1.2 from Theorem 1.3. We apply the lemma with $\mathcal{H}_{1}=\mathcal{L}^{2}\left(\mathbb{R}^{3}\right), \mathcal{H}_{2}=\mathcal{F}, f=e^{-i \int_{0}^{t} \omega(s) d s} \psi_{t}, g=\Omega$,

$$
\Psi=W^{*}\left(\alpha^{2} \varphi_{t}\right) e^{-i \tilde{H}_{\alpha}^{F} t} \psi_{0} \otimes W\left(\alpha^{2} \varphi_{0}\right) \Omega, \quad \Phi=W^{*}\left(\alpha^{2} \varphi_{t}\right) R(t) .
$$

Then Theorem 1.3 implies that the assumptions of the lemma are satisfied with $\varepsilon=\alpha^{-1}(1+|t|)$. We have $\|f\|^{2}=\left\|\psi_{t}\right\|^{2}=\left\|\psi_{0}\right\|^{2}=1,\|g\|^{2}=\|\Omega\|^{2}=1$ and $|f\rangle\left\langle f|=| \psi_{t}\right\rangle\left\langle\psi_{t}\right|$. Moreover,

$$
\operatorname{Tr}_{\mathcal{H}_{2}}|\Psi\rangle\left\langle\Psi\left|=\gamma_{t}^{\text {particle }}, \quad \operatorname{Tr}_{\mathcal{H}_{1}}\right| \Psi\right\rangle\langle\Psi|=W^{*}\left(\alpha^{2} \varphi_{t}\right) \gamma_{t}^{\text {field }} W\left(\alpha^{2} \varphi_{t}\right)
$$

Thus, the conclusion of Theorem 1.2 follows from the lemma.

We now turn to the proof of the lemma. It relies on the bound

$$
\left.\operatorname{Tr}_{\mathcal{H}_{1}}\left|\operatorname{Tr}_{\mathcal{H}_{2}}\right| \Psi_{1}\right\rangle\left\langle\Psi_{2}|| \leq\left\|\Psi_{1}\right\|_{\mathcal{H}_{1} \otimes \mathcal{H}_{2}}\left\|\Psi_{2}\right\|_{\mathcal{H}_{1} \otimes \mathcal{H}_{2}}\right.
$$

valid for any vectors $\Psi_{1}, \Psi_{2} \in \mathcal{H}_{1} \otimes \mathcal{H}_{2}$. For the proof of (D-1) recall the variational characterization of the trace norm,

$$
\operatorname{Tr}_{\mathcal{H}_{1}}|K|=\sup _{\left(e_{j}\right),\left(e_{j}^{\prime}\right)} \operatorname{Re} \sum_{j}\left\langle e_{j}, K e_{j}^{\prime}\right\rangle_{\mathcal{H}_{1}}
$$


where the supremum is over all orthonormal systems $\left(e_{j}\right)$ and $\left(e_{j}^{\prime}\right)$ in $\mathcal{H}_{1}$. Thus, if $\left(b_{k}\right)$ is an orthonormal basis in $\mathcal{H}_{2}$, then

$$
\begin{aligned}
\operatorname{Re} \sum_{j}\left\langle e_{j},\left(\operatorname{Tr}_{\mathcal{H}_{2}}\left|\Psi_{1}\right\rangle\left\langle\Psi_{2}\right|\right) e_{j}^{\prime}\right\rangle_{\mathcal{H}_{1}} & =\operatorname{Re} \sum_{j, k}\left\langle e_{j} \otimes b_{k}, \Psi_{1}\right\rangle_{\mathcal{H}_{1} \otimes \mathcal{H}_{2}}\left\langle\Psi_{2}, e_{j}^{\prime} \otimes b_{k}\right\rangle_{\mathcal{H}_{1} \otimes \mathcal{H}_{2}} \\
& \leq\left(\sum_{j, k}\left|\left\langle e_{j} \otimes b_{k}, \Psi_{1}\right\rangle_{\mathcal{H}_{1} \otimes \mathcal{H}_{2}}\right|^{2}\right)^{\frac{1}{2}}\left(\sum_{j, k}\left|\left\langle\Psi_{2}, e_{j}^{\prime} \otimes b_{k}\right\rangle_{\mathcal{H}_{1} \otimes \mathcal{H}_{2}}\right|^{2}\right)^{\frac{1}{2}} \\
& \leq\left\|\Psi_{1}\right\|_{\mathcal{H}_{1} \otimes \mathcal{H}_{2}}\left\|\Psi_{2}\right\|_{\mathcal{H}_{1} \otimes \mathcal{H}_{2}},
\end{aligned}
$$

where the last inequality comes from the orthonormality of $\left(e_{j} \otimes b_{k}\right)$ and $\left(e_{j}^{\prime} \otimes b_{k}\right)$. Therefore the variational characterization of the trace norm yields (D-1).

Proof. Since $\operatorname{Tr}_{\mathcal{H}_{2}}|f \otimes g\rangle\langle\Phi|=| f\rangle\left\langle\langle g, \Phi\rangle_{\mathcal{H}_{2}}\right|$, we have

$$
\gamma_{1}-\|g\|_{\mathcal{H}_{2}}^{2}|f\rangle\langle f|=| f\rangle\left\langle\langle g, \Phi\rangle_{\mathcal{H}_{2}}|+|\langle\Phi, g\rangle_{\mathcal{H}_{2}}\right\rangle\left\langle f\left|+\operatorname{Tr}_{2}\right| \Phi\right\rangle\langle\Phi| .
$$

By (D-1) and the assumptions the trace norm, each one of the three operators on the right side is bounded by $C^{2} \varepsilon^{2}$. This proves the first inequality in the lemma. The second one is proved similarly.

Finally, we show that the $\alpha^{-2}$ error bound in Theorem 1.2 (for times of order one) is due to the fact that $\varphi_{t}$ is time-dependent. The proof makes use of the fact that for arbitrary normalized vectors $a$ and $b$ in a Hilbert space $\mathcal{H}$ one has

$$
\left.\operatorname{Tr}_{\mathcal{H}}|| a\right\rangle\langle a|-| b\rangle\left\langle b||=2\left(1-|\langle a, b\rangle|^{2}\right)^{\frac{1}{2}}\right.
$$

as is easily verified.

Proof of Lemma 1.4. Because of Theorem 1.2, it suffices to prove that there are $\varepsilon>0$ and $c>0$ such that for all $|t| \leq \varepsilon$ and all $\alpha \geq 1$,

$$
\left.\operatorname{Tr}_{\mathcal{F}}|| W\left(\alpha^{2} \varphi_{t}\right) \Omega\right\rangle\left\langle W\left(\alpha^{2} \varphi_{t}\right) \Omega|-| W\left(\alpha^{2} \varphi_{0}\right) \Omega\right\rangle\left\langle W\left(\alpha^{2} \varphi_{0}\right) \Omega|| \geq c \alpha^{-1}|t| .\right.
$$

According to Lemma A.4 and (D-2), this is equivalent to

$$
1-e^{-\alpha^{2}\left\|\varphi_{t}-\varphi_{0}\right\|_{2}^{2}}=1-\left|\left\langle\Omega, W^{*}\left(\alpha^{2} \varphi_{0}\right) W\left(\alpha^{2} \varphi_{t}\right) \Omega\right\rangle\right|^{2} \geq \frac{1}{4} c^{2} \alpha^{-2} t^{2} .
$$

Since $\left\|\varphi_{t}-\varphi_{0}\right\|_{2} \lesssim \alpha^{-2}|t|$ by Lemma 2.1, it suffices to prove that there are $\varepsilon>0$ and $c^{\prime}>0$ such that for all $|t| \leq \varepsilon$ and all $\alpha \geq 1$,

$$
\left\|\varphi_{t}-\varphi_{0}\right\|_{2} \geq c^{\prime} \alpha^{-2}|t|
$$

Since $\varphi_{0}+\sigma_{\psi_{0}} \not \equiv 0$, this will clearly follow if we can prove that for all $|t| \leq \alpha^{2}$ and $\alpha \geq 1$,

$$
\left\|\varphi_{t}-\varphi_{0}+i \alpha^{-2} t\left(\varphi_{0}+\sigma_{\psi_{0}}\right)\right\|_{2} \leq C \alpha^{-2} t^{2} .
$$

To prove this, we use equation (1-8) for $\varphi_{t}$ to write

$$
\varphi_{t}-\varphi_{0}=\int_{0}^{t} \partial_{s} \varphi_{s} d s=-i \alpha^{-2} \int_{0}^{t}\left(\varphi_{s}+\sigma_{\psi_{s}}\right) d s=-i \alpha^{-2} t\left(\varphi_{0}+\sigma_{\psi_{0}}\right)+r_{t}
$$


with

$$
r_{t}:=-i \alpha^{-2} \int_{0}^{t} \int_{0}^{s}\left(\partial_{s_{1}} \varphi_{s_{1}}+\partial_{s_{1}} \sigma_{\psi_{s_{1}}}\right) d s_{1} d s .
$$

By Lemma 2.1 and Proposition 2.2, the $\mathcal{L}^{2}$-norm of the integrand of $r_{t}$ is bounded by a constant uniformly in $\left|s_{1}\right| \leq \alpha^{2}$ and $\alpha \geq 1$. This yields (D-3) and completes the proof.

\section{Appendix E: Improving the result of [Frank and Schlein 2014]}

We now show how the techniques from [Frank and Schlein 2014] can be extended to times $|t|=o(\alpha)$. This argument is due to an anonymous referee, whom we thank for kind permission to include it in our paper.

Proposition E.1. Let $\varphi \in L^{2}\left(\mathbb{R}^{3}\right)$ and $\alpha_{0}>0$. Assume that $\Psi \in L^{2}\left(\mathbb{R}^{3}\right) \otimes \mathcal{F}$ satisfies

$$
\left\|\left(p^{2}+\mathcal{N}+1\right)^{\frac{1}{2}} \Psi\right\| \leq M, \quad\left\|\left(p^{2}+1\right)^{\frac{1}{2}} \mathcal{N} \Psi\right\| \leq M \alpha^{-2} .
$$

Then for all $\alpha \geq \alpha_{0}$ and all $t \in \mathbb{R}$,

$$
\left\|e^{-i \tilde{H}_{\alpha}^{F} t} W\left(\alpha^{2} \varphi\right) \Psi-e^{-i H_{\varphi} t} W\left(\alpha^{2} \varphi\right) \Psi\right\|^{2} \leq M^{2}\left(1+2 \alpha^{-1}\right)\left(e^{C|t| /(2 \alpha)}-1\right),
$$

where $C$ depends only on $\alpha_{0}$ and an upper bound on $\|\varphi\|_{\mathcal{L}^{2}}$.

Note that this result can be applied, in particular, to $\Psi=\psi \otimes \Omega$ with $\|\psi\|_{\mathcal{H}^{1}} \leq M$. We also recall that the effective Schrödinger operator $H_{\varphi}$ was defined in (2-12).

Proof. Let $A(t):=\left\|e^{-i \tilde{H}_{\alpha}^{\mathrm{F}} t} W\left(\alpha^{2} \varphi\right) \Psi-e^{-i H_{\varphi} t} W\left(\alpha^{2} \varphi\right) \Psi\right\|^{2}$. It is shown in [Frank and Schlein 2014, Proposition 9] that $A^{\prime}(t)=f(t)+g(t)$ with

$$
f(t) \leq C M \alpha^{-1} A(t)^{\frac{1}{2}}, \quad \int_{0}^{T} g(t) d t \leq C M^{2} \alpha^{-2} T,
$$

where $C$ depends only on $\alpha_{0}$ and an upper bound on $\|\varphi\|_{\mathcal{L}^{2}}$. We bound $f(t) \leq \frac{1}{2} C \alpha^{-1}\left(A(t)+M^{2}\right)$ and therefore

Thus,

$$
A(T) \leq \int_{0}^{T} f(t) d t+\int_{0}^{T} g(t) d t \leq \frac{1}{2} C \alpha^{-1} \int_{0}^{T} A(t) d t+\frac{1}{2} C M^{2} \alpha^{-1}\left(1+2 \alpha^{-1}\right) T .
$$

$$
A(T)+M^{2}\left(1+2 \alpha^{-1}\right) \leq M^{2}\left(1+2 \alpha^{-1}\right)+\frac{1}{2} C \alpha^{-1} \int_{0}^{T}\left(A(t)+M^{2}\left(1+2 \alpha^{-1}\right)\right) d t
$$

and, by Gronwall's inequality, for all $t \geq 0$

$$
A(t)+M^{2}\left(1+2 \alpha^{-1}\right) \leq M^{2}\left(1+2 \alpha^{-1}\right) e^{C t /(2 \alpha)} .
$$

\section{Acknowledgements}

The authors are grateful to J. Fröhlich, M. Lewin, B. Schlein and R. Seiringer for their helpful remarks at various stages of this project, as well as to the anonymous referees who helped improve this paper. Support through NSF grants PHY-1347399 and DMS-1363432 (R.L.F.) and DMS-1308985 and DMS-1443225 (Z.G.) is acknowledged. 


\section{Note added in proof}

After this work was accepted for publication, the preprint by M. Griesemer [2016] appeared on the arXiv. This preprint studies the dynamics generated by the initial conditions given by the minimizing pair $\left(\psi_{*}, \varphi_{*}\right)$ of the energy functional $\mathcal{E}(\psi, \varphi)$ under the constraint $\|\psi\|=1$ up to times of order $o\left(\alpha^{2}\right)$.

\section{References}

[Ammari and Falconi 2014] Z. Ammari and M. Falconi, "Wigner measures approach to the classical limit of the Nelson model: convergence of dynamics and ground state energy", J. Stat. Phys. 157:2 (2014), 330-362. MR Zbl

[Ammari and Nier 2008] Z. Ammari and F. Nier, "Mean field limit for bosons and infinite dimensional phase-space analysis", Ann. Henri Poincaré 9:8 (2008), 1503-1574. MR Zbl

[Ammari and Nier 2009] Z. Ammari and F. Nier, "Mean field limit for bosons and propagation of Wigner measures", J. Math. Phys. 50:4 (2009), art. id. 042107. MR Zbl

[Devreese and Alexandrov 2009] J. T. Devreese and A. S. Alexandrov, "Fröhlich polaron and bipolaron: recent developments", Rep. Prog. Phys. 72:6 (2009), art. id. 066501.

[Donsker and Varadhan 1983] M. D. Donsker and S. R. S. Varadhan, "Asymptotics for the polaron", Comm. Pure Appl. Math. 36:4 (1983), 505-528. MR Zbl

[Falconi 2013] M. Falconi, "Classical limit of the Nelson model with cutoff”, J. Math. Phys. 54:1 (2013), art. id. 012303. MR $\mathrm{Zbl}$

[Frank and Schlein 2014] R. L. Frank and B. Schlein, "Dynamics of a strongly coupled polaron”, Lett. Math. Phys. 104:8 (2014), 911-929. MR Zbl

[Fröhlich 1937] H. Fröhlich, "Theory of electrical breakdown in ionic crystals", Proc. R. Soc. Lond. A 160:901 (1937), $230-241$.

[Ginibre et al. 2006] J. Ginibre, F. Nironi, and G. Velo, "Partially classical limit of the Nelson model", Ann. Henri Poincaré 7:1 (2006), 21-43. MR Zbl

[Griesemer 2016] M. Griesemer, "On the dynamics of polarons in the strong-coupling limit", preprint, 2016. arXiv

[Hepp 1974] K. Hepp, "The classical limit for quantum mechanical correlation functions", Comm. Math. Phys. 35:4 (1974), 265-277. MR

[Landau and Pekar 1948] L. D. Landau and S. I. Pekar, "Effective mass of a polaron”, Zh. Eksp. Teor. Fiz. 18:5 (1948), 419-423. In Russian.

[Lieb and Thomas 1997] E. H. Lieb and L. E. Thomas, "Exact ground state energy of the strong-coupling polaron", Comm. Math. Phys. 183:3 (1997), 511-519. MR Zbl

[Lieb and Yamazaki 1958] E. H. Lieb and K. Yamazaki, "Ground-state energy and effective mass of the polaron", Phys. Rev. 111:3 (1958), 728-733. Zbl

[Pekar 1946] S. Pekar, "Avto lokalizatsiya èlektrona v dièlektricheskoŭ polyarizuyushcheřsya srede", Zh. Eksp. Teor. Fiz. 16:4 (1946), 335-340.

[Pekar 1951] S. Pekar, Issledovaniya po èlektronnoŭ teorii kristallov, Gostekhizdat, Moscow, 1951. Translated as Research in electron theory of crystals, US Atomic Energy Com., Oak Ridge, TN, 1963.

[Spohn 1987] H. Spohn, "Effective mass of the polaron: a functional integral approach", Ann. Physics 175:2 (1987), 278-318. MR

Received 30 Jun 2016. Accepted 28 Nov 2016.

RUPERT L. FRANK: rlfrank@caltech.edu

Mathematics 253-37, Caltech, Pasadena, CA 91125, United States

ZHOU GANG: gzhou@caltech.edu

Mathematics 253-37, Caltech, Pasadena, CA 91125, United States 